\title{
Extremal Eigenvalues and Eigenvectors of Deformed Wigner Matrices
}

\author{
Ji Oon Lee \\ Department of Mathematical Sciences \\ Korea Advanced Institute of Science and Technology \\ jioon.lee@kaist.edu
}

\author{
Kevin Schnelli \\ School of Mathematics \\ Institute for Advanced Study \\ kschnelli@math.ias.edu
}

\begin{abstract}
We consider random matrices of the form $H=W+\lambda V, \lambda \in \mathbb{R}^{+}$, where $W$ is a real symmetric or complex Hermitian Wigner matrix of size $N$ and $V$ is a real bounded diagonal random matrix of size $N$ with i.i.d. entries that are independent of $W$. We assume subexponential decay of the distribution of the matrix entries of $W$ and we choose $\lambda \sim 1$, so that the eigenvalues of $W$ and $\lambda V$ are typically of the same order. Further, we assume that the density of the entries of $V$ is supported on a single interval and is convex near the edges of its support. In this paper we prove that there is $\lambda_{+} \in \mathbb{R}^{+}$such that the largest eigenvalues of $H$ are in the limit of large $N$ determined by the order statistics of $V$ for $\lambda>\lambda_{+}$. In particular, the largest eigenvalue of $H$ has a Weibull distribution in the limit $N \rightarrow \infty$ if $\lambda>\lambda_{+}$. Moreover, for $N$ sufficiently large, we show that the eigenvectors associated to the largest eigenvalues are partially localized for $\lambda>\lambda_{+}$, while they are completely delocalized for $\lambda<\lambda_{+}$. Similar results hold for the lowest eigenvalues.
\end{abstract}

AMS Subject Classification (2010): 15B52,60B20, 82B44

Keywords: Random matrix, Local semicircle law, Delocalization, Localization

22nd June 2018

\section{INTRODUCTION}

The universality of random matrices is usually divided into bulk and edge universalities. Edge universality concerns the distribution of the extreme eigenvalues. It is known that the extreme eigenvalues of a large class of Wigner matrices exhibit universal limiting behavior. The limiting distribution of the largest eigenvalue was first identified by Tracy and Widom [49, 50 for the Gaussian ensembles. Edge universality for Wigner matrices has first been proved by Soshnikov [46] (see also 45]) for real symmetric and complex Hermitian ensembles with symmetric distributions. The symmetry assumption on the entries' distribution was partially removed in 40, 41. Edge universality without any symmetry assumption was proved in [48] under the condition that the distribution of the matrix elements has subexponential decay and its first three moments match those of the Gaussian distribution. For Wigner matrices with arbitrary symmetry class, edge universality was proven in 15. under the assumption that the entries have $12+\epsilon$ moments. Recently, a necessary and sufficient condition for the edge universality of Wigner matrices was given in 33.

The distribution of the largest eigenvalues of a random diagonal matrix $V$ whose entries $\left(v_{i}\right)$ are i.i.d. real random variables is given by the order statistics of $\left(v_{i}\right)$. The Fisher-Tippett-Gnedenko theorem (see e.g. [27]) thus implies that the limiting distribution of the largest eigenvalue of $V$ belongs either to the Gumbel, Fréchet or Weibull family.

In this paper, we consider the interpolation between Wigner matrices and real diagonal random matrices. Let $W$ be an $N \times N$ real symmetric or complex Hermitian Wigner matrix whose centered entries have variance $N^{-1}$ and 
subexponential decay. Let $V$ be an $N \times N$ real diagonal random matrix whose entries are bounded i.i.d. random variables. For $\lambda \in \mathbb{R}^{+}$we set

$$
H=\left(h_{i j}\right):=\lambda V+W, \quad(1 \leq i, j \leq N)
$$

The matrices $V$ and $W$ are normalized in the sense that the eigenvalues of $V$ and $W$ are of order one.

If $W$ belongs to the Gaussian Unitary ensemble (GUE), the model (1.1) is called the deformed GUE. It was shown in 29, 43 that the edge eigenvalues of the deformed GUE are governed by the Tracy-Widom distribution for $\lambda \ll N^{-1 / 6}$. At $\lambda \sim N^{-1 / 6}$ the fluctuations of the edge eigenvalues change from the Tracy-Widom to a Gaussian distribution. More precisely, Johansson showed in 29] that the limiting distribution of the edge eigenvalues for $\lambda=\alpha N^{-1 / 6}$ is given by the convolution of the Tracy-Widom and the centered Gaussian distribution, with variance depending on $\alpha$. These results have not been established for the Gaussian orthogonal ensemble (GOE) or for general Wigner matrices.

In the present paper, we consider the edge behavior of the deformed model (1.1) in the regime $\lambda \sim 1$ with $W$ a real symmetric or complex Hermitian matrix. We show that there is, for certain $V$, yet another transition for the limiting behavior of the largest eigenvalues of $H$ as $\lambda$ varies. For simplicity, we assume that the distribution of the entries of $V$ is centered and given by the density

$$
\mu(v):=Z^{-1}(1+v)^{\mathrm{a}}(1-v)^{\mathrm{b}} d(v) \mathbb{1}_{[-1,1]}(v),
$$

where $-1 \leq \mathrm{a}, \mathrm{b}<\infty, d$ is a strictly positive $C^{1}$-function and $Z$ is a normalization constant. We primarily focus on the choices a, $\mathrm{b}>1$. From our first main result, Theorem 2.8 , it follows that there are $N$-independent constants $\lambda_{+} \equiv \lambda_{+}(\mu)>1$ and $L_{+} \equiv L_{+}(\mu, \lambda)>2$, such that, for $\mathrm{b}>1$ and $\lambda>\lambda_{+}$, the largest eigenvalue $\mu_{1}$ of $H$ satisfies

$$
\lim _{N \rightarrow \infty} \mathbb{P}\left(N^{1 /(\mathrm{b}+1)}\left(L_{+}-\mu_{1}\right) \leq x\right)=G_{\mathrm{b}+1}(x), \quad \mathrm{b}>1, \quad \lambda>\lambda_{+},
$$

where $G_{\mathrm{b}+1}$ is a Weibull distribution with parameter $\mathrm{b}+1$; see (2.20).

However, if $\lambda<\lambda_{+}$, then there are $N$-independent constants $L_{+} \equiv L_{+}(\mu, \lambda)$ and $c \equiv c(\mu, \lambda)$, such that

$$
\lim _{N \rightarrow \infty} \mathbb{P}\left(N^{1 / 2}\left(L_{+}-\mu_{1}\right) \leq x\right)=\Phi_{c}(x), \quad \mathrm{b}>1, \quad \lambda<\lambda_{+},
$$

where $\Phi_{c}$ denotes the cumulative distribution function of the centered Gaussian distribution with variance $c$; see Appendix C. We remark that neither (1.3) nor (1.4) depend on the symmetry type of the Wigner matrix $W$.

The appearance of the Weibull distribution in the model (1.1) is indeed expected when $\lambda$ grows sufficiently fast with $N$, since in this case the diagonal matrix dominates the spectral properties of $H$. However, it is quite surprising that the Weibull distribution already appears for $\lambda$ order one, since the local behavior of the eigenvalues in the bulk of the deformed model mainly stems from the Wigner part, and the contribution from the random diagonal part is limited to mesoscopic fluctuations of the eigenvalues; see [32].

Having identified two possible limiting distributions of the largest eigenvalues, it is natural to ask about the behavior of the associated eigenvectors. Before considering the deformed model, we recall that the eigenvectors of Wigner matrices with subexponential decay are completely delocalized, as was proved by Erdős, Schlein and Yau [20, 21].

For deformed Wigner matrices we show that the eigenvectors of the largest eigenvalues are partially localized in the regime where the edge behavior (1.3) holds. More precisely, we prove that one component of the $\left(\ell^{2}-\right.$ normalized) eigenvectors associated with eigenvalues at the extreme edge carries a weight of order one, while the other components each carry a weight of order $o(1)$; see Theorem 2.11, If, however, the edge behavior (1.4) holds, all eigenvectors are completely delocalized. Although we do not prove it explicitly, we claim that the bulk eigenvectors of the model (1.1) with (1.2) for the choice of $\mu$, are completely delocalized (for any choice of $\lambda \sim 1$ ). This can be proved with the very same methods as in [32. To understand the transition from partial localization to delocalization, further efforts are required.

The phenomenology described above is reminiscent of the one for heavy-tailed Wigner matrices. For instance, consider real symmetric Wigner matrices whose entries' distribution function decays as a power law, i.e.,

$$
\mathbb{P}\left(\left|h_{i j}\right|>x\right)=L(x) x^{-\alpha}, \quad(1 \leq i, j \leq N),
$$

for some slowly varying function $L(x)$. It was proved by Soshnikov 47] that the linear statistics of the largest eigenvalues are Poissonian for $\alpha<2$; in particular, the largest eigenvalue has a Fréchet limit distribution. Later, Auffinger, Ben Arous and Péché [3] showed that the same conclusions hold for $2 \leq \alpha<4$ as well. Recently, it was proved by Bordenave and Guionnet [8] that the eigenvectors of models satisfying (1.5) are weakly delocalized for $1<\alpha<2$. For $0<\alpha<1$, it is conjectured [10] that there is a sharp "metal-insulator" transition. In [8] it is proved that the eigenvectors of sufficiently large eigenvalues are weakly localized for $0<\alpha<2 / 3$.

To clarify the terminology "partial localization" we remark that it is quite different from the usual notion of localization for random Schrödinger operators. The telltale signature of localization for random Schrödinger 
operators is an exponential decay of off-diagonal Green function entries, which implies the absence of diffusion, spectral localization etc. For the Anderson model in dimensions $d \geq 3$ such an exponential decay was first obtained by Fröhlich and Spencer [26] using a multiscale analysis. Later, a similar bound was presented by Aizenman and Molchanov 1 using fractional moments. Due to the mean-field nature of the Wigner matrix $W$, there is no notion of distance for the deformed model (1.1). Instead, our localization result states that most of the mass of the eigenvectors is concentrated on a few sites, whose locations are independent and uniformly distributed. This result agrees with the predictions of formal perturbation theory.

Yet, there are some similarities with the Anderson model in $d \geq 3$ : In the Anderson model localization occurs where the density of states is (exponentially) small [26]; this is known to happen close to the spectral edges or for large disorder. Further it is strongly believed that the Anderson model admits extended states, i.e., the generalized eigenvectors in the bulk are expected to be delocalized. Moreover, it was proven by Minami [31] that the local eigenvalue statistics of the Anderson model can be described by a Poisson point process in the strong localization regime. It is also conjectured that the local eigenvalue statistics in the bulk are given by the GOE statistics, respectively GUE statistics if time-reversal symmetry is broken. We remark that there are some partial results on bulk universality for the deformed model (1.1); see [43, 37.

We also mention that the localization result we prove in this paper also differs from that for random band matrices, where all the eigenvectors are localized, even in the bulk. We refer to [42, 13, 16] for more details on the localization/delocalization for random band matrices.

Next, we outline the proofs of our main results. It was first shown by Pastur 38 that the empirical eigenvalue distribution of the deformed model (1.1) converges to a deterministic distribution in the limit $N \rightarrow \infty$ under some weak assumption on $\lambda V$. However, this limiting eigenvalue distribution, referred to as the deformed semicircle law in the following, is in general different from Wigner's semicircle law and depends on the limiting distribution of $\lambda V$. The deformed semicircle law can be defined in terms of a functional equation for the Stieltjes transforms of the limiting eigenvalue distributions of $\lambda V$ and $W$ 38. Restricting the discussion to the special case when the entries of $V$ follow the centered Jacobi distribution in (1.2) with $\mathrm{b}>1$, we showed in 32 that the deformed semicircle law, henceforth denoted by $\mu_{f c}$, is supported on a single interval and shows either of the following behavior close to the upper edge:

$$
\mu_{f c}(E) \sim \begin{cases}\sqrt{\kappa_{E}}, & \text { for } \quad \lambda<\lambda_{+} \\ \left(\kappa_{E}\right)^{\mathrm{b}}, & \text { for } \quad \lambda>\lambda_{+}\end{cases}
$$

for $E \in \operatorname{supp} \mu_{f c}, E \geq 0$, where $\kappa_{E}$ denotes the distance from $E$ to the upper endpoint of the support of $\mu_{f c}$; see Lemma 2.4 below. In case the square root behavior prevails, we are going to show that the largest eigenvalue of $H$ satisfies (1.4), whereas in case we have a "convex decay" with b > 1, (1.3) is satisfied.

In a first step, we derive a local law for the empirical eigenvalue density: Under some moment conditions the convergence of the empirical eigenvalue distribution to the semicircle law also holds on very small scales. Denoting by $G(z)=(H-z)^{-1}, z \in \mathbb{C}^{+}$, the Green function or resolvent of $H$, convergence of the empirical eigenvalue distribution on scale $\eta$ around an energy $E \in \mathbb{R}$ is equivalent to the convergence of the averaged Green function $m(z)=N^{-1} \operatorname{Tr} G(z), z=E+\mathrm{i} \eta$. In a series of papers [20, 21, 22] Erdős, Schlein and Yau showed that the semicircle law for Wigner matrices also holds down to the optimal scale $1 / N$, up to logarithmic corrections. In 24 . a "fluctuation average lemma" was introduced that yielded optimal bounds on the convergence of $m(z)$ for Wigner matrices in the bulk 24] and up to the edge [25] on scales $\eta \gg N^{-1}$. Below this scale the eigenvalue density remains fluctuating even for large $N$. In 25 the Green function $G(z)$ and its average $m(z)$ have been used to prove edge universality for generalized Wigner matrices. In 32 we derived a local deformed semicircle law for the deformed ensemble (1.1) under the assumption that $\mu_{f c}$ has a square root behavior at the endpoints. In the present paper, we derive a local law at the extreme edge in case $\mu_{f c}$ shows a convex decay at the edge. We propose, however, a slightly different path than the one taken in 32: We condition on the random variables $\left(v_{i}\right)$ and show that $m(z)$ converges, for "typical" realizations of $\left(v_{i}\right)$, on scale $\sim N^{-1 / 2}$; see Proposition [5.1] In particular, we show that the typical eigenvalue spacing at the extreme edge is of order $N^{-1 /(\mathrm{b}+1)} \gg N^{-1 / 2}$; as is suggested by the convex decay in (1.6). Similar to the Wigner case, see e.g., [25, this is accomplished by deriving a self-consistent equation for $m(z)$. However, the analysis of the self-consistent equation is quite different from the Wigner case, due to the absence of the usual stability bound; see 32 .

In a second step, we can use the self-averaging property of the Wigner matrix $W$, to show that the imaginary part of $m$ can be controlled on scales much smaller than $N^{-1 / 2}$ : the technical input here is the "fluctuation averaging lemma" [25, 18, 17]. Our proof relies on the basic strategy of [17]. However, in our setup the diagonal entries of $G$ are not uniformly bounded, which requires several changes to previous arguments. To complete the proof of our first main result, Theorem 2.11, we note that the imaginary part of $m$ can be written as

$$
\operatorname{Im} m(E+\mathrm{i} \eta)=\frac{1}{N} \sum_{\alpha=1}^{N} \frac{\eta}{\left(\mu_{\alpha}-E\right)^{2}+\eta^{2}}, \quad(E \in \mathbb{R}, \eta>0),
$$


where $\left(\mu_{\alpha}\right)$ are the eigenvalues of $H$. Thus, having control on the left side for $\eta \ll N^{-1 / 2}$ allows tracking the individual eigenvalues at the extreme edge, where their typical spacing much bigger than $N^{-1 / 2}$.

Finally, we point out the main steps in the proof of the partial localization of eigenvectors; see Theorem 2.11 for precise results. It is well-known that information on the averaged Green function $m(z)$ can be translated via the Helffer-Sjöstrand formula to information on the density of states; see, e.g., [19]. Since the typical eigenvalue spacing at the edge is, for the case at hand, much larger than $N^{-1 / 2}$, the Helffer-Sjöstrand formula also allows to translate information on the diagonal Green function entries $\left(G_{i i}(z)\right)$ into information on the eigenvectors at the edge. Relying on estimates on the Green function, we can then prove "partial localization" of the eigenvectors at the edge.

The paper is organized as follows: In Section 2, we introduce the precise definition of the model and state the main results of the paper. In Section 3, we collect basic notations and identities for the resolvent of $H$. In Section 4 , we prove the first main result of the paper, Theorem 2.8, using estimates on the Stieltjes transform of the deformed semicircle measure. (See also Corollary 4.9.) In Sections 5 and [6 we prove important lemmas on the location of the extreme eigenvalues, including the local law, which have crucial roles in the proof of Theorem 2.8. In Section 7. we prove the second main result of the paper, Theorem 2.11, on the partial localization of the eigenvectors at the edge. Proofs of some technical lemmas are collected in the Appendices A, B and C.

Acknowledgements: We thank Horng-Tzer Yau for numerous helpful discussions and remarks. We are also grateful to Paul Bourgade, László Erdős and Antti Knowles for discussions and comments. Ji Oon Lee is partially supported by the Basic Science Research Program of the National Research Foundation of Korea, Grant 20110013474. The stay of Kevin Schnelli at IAS is supported by The Fund For Math.

\section{Definition And Results}

In this section, we define our model and state our main results.

2.1. Deformed semicircle law. For a (probability) measure, $\omega$, on $\mathbb{R}$, we define its Stieltjes transform by

$$
m_{\omega}(z):=\int_{\mathbb{R}} \frac{\mathrm{d} \omega(x)}{x-z}, \quad\left(z \in \mathbb{C}^{+}\right) .
$$

Note that $m_{\omega}(z)$ is an analytic function in the upper half plane, satisfying $\operatorname{Im} m_{\omega}(z) \geq 0, z \in \mathbb{C}^{+}$.

As first shown in [38, the Stieltjes transform of the limiting spectral distribution of the interpolating model (1.1) satisfies the equation

$$
m_{f c}(z)=\int_{\mathbb{R}} \frac{\mathrm{d} \mu(x)}{\lambda v-z-m_{f c}(z)}, \quad \operatorname{Im} m_{f c}(z) \geq 0, \quad\left(z \in \mathbb{C}^{+}\right),
$$

where $\mu$ is the distribution of the i.i.d. random variables $\left(v_{i}\right)$. Equation (2.2) is often called the Pastur relation. It is shown in [38, 7] that (2.2) has a unique solution. Moreover, it is easy to check that $\lim \sup _{\eta \searrow 0} \operatorname{Im} m_{f c}(E+\mathrm{i} \eta)<\infty$, thus $m_{f c}(z)$ determines an absolutely continuous probability measure on $\mathbb{R}$, whose density, $\mu_{f c}$, is given by

$$
\mu_{f c}(E)=\frac{1}{\pi} \lim _{\eta \searrow 0} \operatorname{Im} m_{f c}(E+\mathrm{i} \eta), \quad(E \in \mathbb{R}) .
$$

The measure $\mu_{f c}$ has been studied in details in [7]; for example, it was shown that $\mu_{f c}$ is an analytic function inside its support.

Remark 2.1. Setting $\lambda=0$, (2.2) reduces to

$$
m_{f c}(z)=-\frac{1}{z+m_{f c}(z)}, \quad \operatorname{Im} m_{f c}(z) \geq 0, \quad\left(z \in \mathbb{C}^{+}\right),
$$

and one immediately checks that in this case $\mu_{f c}$ is given, as expected, by the standard semicircular measure, $\mu_{s c}$, which is characterized by the density $\mu_{s c}(E)=\frac{1}{2 \pi} \sqrt{\left(4-E^{2}\right)_{+}}$.

Remark 2.2. The measure $\mu_{f c}$ is often called the additive free convolution of the semicircular law and the measure $\mu$ (up to the scaling by $\lambda$ ). More generally, the additive free convolution of two (probability) measures $\omega_{1}$ and $\omega_{2}$, usually denoted by $\omega_{1} \boxplus \omega_{2}$, is defined as the distribution of the sum of two freely independent non-commutative random variables, having distributions $\omega_{1}, \omega_{2}$ respectively; we refer to [51, 35, 28, 22. Similarly to (2.2), the free convolution measure $\omega_{1} \boxplus \omega_{2}$ can be described in terms of a set of functional equations for the Stieltjes transforms; see [39, 11, 5]. For a discussion of regularity properties of $\omega_{1} \boxplus \omega_{2}$ we refer to [4].

Free probability theory turned out to be a natural setting for studying global laws for such ensembles; see, e.g., [51, 2]. For more recent treatments, including local laws, we refer to [30, 9, 6]. 


\subsection{Definition of the model.}

Definition 2.3. Let $W$ be an $N \times N$ random matrix, whose entries, $\left(w_{i j}\right)$, are independent, up to the symmetry constraint $w_{i j}=\overline{w_{j i}}$, centered, real (complex) random variables with variance $N^{-1}$ and with subexponential decay, i.e.,

$$
\mathbb{P}\left(\sqrt{N}\left|w_{i j}\right|>x\right) \leq C_{0} \mathrm{e}^{-x^{1 / \theta}}
$$

for some positive constants $C_{0}$ and $\theta>1$. In particular, if $\left(w_{i j}\right)$ are complex random variables,

$$
\mathbb{E} w_{i j}=0, \quad \mathbb{E}\left|w_{i j}\right|^{2}=\frac{1}{N}, \quad \mathbb{E} w_{i j}^{2}=0, \quad \mathbb{E}\left|w_{i j}\right|^{p} \leq C \frac{(\theta p)^{\theta p}}{N^{p / 2}}, \quad(p \geq 3)
$$

if $\left(w_{i j}\right)$ are real random variables,

$$
\mathbb{E} w_{i j}=0, \quad \mathbb{E} w_{i j}^{2}=\frac{1+\delta_{i j}}{N}, \quad \mathbb{E}\left|w_{i j}\right|^{p} \leq C \frac{(\theta p)^{\theta p}}{N^{p / 2}}, \quad(p \geq 3) .
$$

Let $V$ be an $N \times N$ diagonal random matrix, whose entries $\left(v_{i}\right)$ are real, centered, i.i.d. random variables, independent of $W=\left(w_{i j}\right)$, with law $\mu$. More assumptions on $\mu$ will be stated below. Without loss of generality, we assume that the entries of $V$ are ordered,

$$
v_{1} \geq v_{2} \geq \ldots \geq v_{N}
$$

For $\lambda \in \mathbb{R}^{+}$, we consider the random matrix

$$
H=\left(h_{i j}\right):=\lambda V+W
$$

We choose for simplicity $\mu$ as a Jacobi measure, i.e., $\mu$ is described in terms of its density

$$
\mu(v)=Z^{-1}(1+v)^{\mathrm{a}}(1-v)^{\mathrm{b}} d(v) \mathbb{1}_{[-1,1]}(v),
$$

where a, b $>-1, d \in C^{1}([-1,1])$ such that $d(v)>0, v \in[-1,1]$, and $Z$ is an appropriately chosen normalization constant. We assume, for simplicity of the arguments, that $\mu$ is centered, but this condition can easily be relaxed. We remark that the measure $\mu$ has support $[-1,1]$, but we observe that varying $\lambda$ is equivalent to changing the support of $\mu$. Since $\mu$ is absolutely continuous, we may assume that (2.8) holds with strict inequalities.

2.3. Edge behavior of $\mu_{f c}$. Properties of $\mu_{f c}$ with the special choice (2.10) for $\mu$ and with $\lambda \sim 1$, have been studied in [32]; see also [7, 44]. For example, the support of $\mu_{f c}$ consists of a single interval. For $E \in \mathbb{R}$, we denote by $\kappa_{E}$ the distance to the endpoints support of $\mu_{f c}$, i.e.,

$$
\kappa_{E}:=\min \left\{\left|E-L_{-}\right|,\left|E-L_{+}\right|\right\}, \quad \operatorname{supp} \mu_{f c}=\left[L_{-}, L_{+}\right], \quad(E \in \mathbb{R}) .
$$

In the following we will often abbreviate $\kappa \equiv \kappa_{E}$.

In the present paper, we are mainly interested in the limiting behavior of the largest, respectively smallest, eigenvalues of the interpolating matrix (2.9), for $\lambda \sim 1$. For concreteness, we focus on the upper edge and comment on the lower edge in Remark 2.6. The following lemma is taken from [32]; see also [7, 44, 36] for statement (1).

Lemma 2.4. Let $\mu$ be a centered Jacobi measure defined in (2.10) with $\mathrm{b}>1$. Define

$$
\lambda_{+}:=\left(\int_{-1}^{1} \frac{\mu(v) \mathrm{d} v}{(1-v)^{2}}\right)^{1 / 2}, \quad \tau_{+}:=\int_{-1}^{1} \frac{\mu(v) \mathrm{d} v}{1-v} .
$$

Then, there exist $L_{-}<0<L_{+}$such that the support of $\mu_{f c}$ is $\left[L_{-}, L_{+}\right]$. Moreover,

(1) if $\lambda<\lambda_{+}$, then for $0 \leq \kappa \leq L_{+}$,

$$
C^{-1} \sqrt{\kappa} \leq \mu_{f c}\left(L_{+}-\kappa\right) \leq C \sqrt{\kappa}
$$

for some $C \geq 1$;

(2) if $\lambda>\lambda_{+}$, then $L_{+}=\lambda+\left(\tau_{+} / \lambda\right)$ and, for $0 \leq \kappa \leq L_{+}$,

$$
C^{-1} \kappa^{\mathrm{b}} \leq \mu_{f c}\left(L_{+}-\kappa\right) \leq C \kappa^{\mathrm{b}},
$$

for some $C \geq 1$. Moreover, $L_{+}$satisfies $L_{+}+m_{f c}\left(L_{+}\right)=\lambda$. 
Remark 2.5. Since

$$
\tau_{+}=\int_{-1}^{1} \frac{\mu(v) \mathrm{d} v}{1-v}>\int_{-1}^{1}(1+v) \mu(v) \mathrm{d} v=1,
$$

we find that $L_{+}>\lambda+(1 / \lambda) \geq 2$. Similarly, we also have that $L_{-} \leq-2$.

Remark 2.6. For a $>1$, the analogue statements to Lemma 2.4 hold for the lower endpoint $L_{-}$of the support of $\mu_{f c}$, with $\lambda_{+}$and $\tau_{+}$replaced by

$$
\lambda_{-}:=\left(\int_{-1}^{1} \frac{\mu(v) \mathrm{d} v}{(1+v)^{2}}\right)^{1 / 2}, \quad \tau_{-}:=\int_{-1}^{1} \frac{\mu(v) \mathrm{d} v}{1+v} .
$$

Remark 2.7. When $-1<\mathrm{a}, \mathrm{b}<1$, there exist, for any $\lambda \in \mathbb{R}^{+}, L_{-}<0<L_{+}$, such that $\operatorname{supp} \mu_{f c}=\left[L_{-}, L_{+}\right]$. Moreover, for any $\lambda \in \mathbb{R}^{+}$, there exists $C \geq 1$ such that

$$
C^{-1} \sqrt{\kappa_{E}} \leq \mu_{f c}(E) \leq C \sqrt{\kappa_{E}}, \quad E \in\left[L_{-}, L_{+}\right]
$$

If $\mathrm{a}<1<\mathrm{b}$ or $\mathrm{b}<1<\mathrm{a}$, the analogous statement to (2.17) holds only at the lower edge or at the upper edge, respectively. These results can be proved using the methods of [44]; see [32] for more details.

In 32 , spectral properties of the interpolating matrix (2.9) have been analyzed in detail under the assumption that (2.17) holds, i.e., it was assumed that either $\lambda$ is sufficiently small or $\mathrm{a}, \mathrm{b} \leq 1$.

2.4. Main results. Denote by $\left(\mu_{i}\right)$ the ordered eigenvalues of the matrix $H=\lambda V+W$,

$$
\mu_{1} \geq \mu_{2} \geq \ldots \geq \mu_{N}
$$

In the following, we fix some $n_{0} \in \mathbb{N}$, independent of $N$, and consider the largest eigenvalues $\left(\mu_{i}\right)_{i=1}^{n_{0}}$ of $H$. All our results also apply mutatis mutandis to the smallest eigenvalues $\left(\mu_{i}\right)_{i=N-n_{0}}^{N}$ of $H$ as can readily be checked.

2.4.1. Eigenvalue statistics. The first main result of the paper shows that the locations of the extreme eigenvalues are determined by the order statistics of the diagonal elements $\left(v_{i}\right)$. Recall that we denote by $\mu$ the distribution of the (unordered) centered random variables $\left(v_{i}\right)$.

Theorem 2.8. Let $W$ be a real symmetric or complex Hermitian Wigner matrix, satisfying the assumptions in Definition 2.3. Assume that the distribution $\mu$ is given by (2.10) with $b>1$ and fix some $\lambda>\lambda_{+} ;$see (2.12). Let $n_{0}>10$ be a fixed constant independent of $N$, denote by $\mu_{i}$ the $i$-th largest eigenvalue of $H=\lambda V+W$ and let $1 \leq k<n_{0}$. Then the joint distribution function of the $k$ largest rescaled eigenvalues,

$$
\mathbb{P}\left(N^{1 /(\mathrm{b}+1)}\left(L_{+}-\mu_{1}\right) \leq s_{1}, N^{1 /(\mathrm{b}+1)}\left(L_{+}-\mu_{2}\right) \leq s_{2}, \ldots, N^{1 /(\mathrm{b}+1)}\left(L_{+}-\mu_{k}\right) \leq s_{k}\right)
$$

converges to the joint distribution function of the $k$ largest rescaled order statistics of $\left(v_{i}\right)$,

$$
\mathbb{P}\left(C_{\lambda} N^{1 /(\mathrm{b}+1)}\left(1-v_{1}\right) \leq s_{1}, C_{\lambda} N^{1 /(\mathrm{b}+1)}\left(1-v_{2}\right) \leq s_{2}, \ldots, C_{\lambda} N^{1 /(\mathrm{b}+1)}\left(1-v_{k}\right) \leq s_{k}\right),
$$

as $N \rightarrow \infty$, where $C_{\lambda}=\frac{\lambda^{2}-\lambda_{+}^{2}}{\lambda}$. In particular, the cumulative distribution function of the rescaled largest eigenvalue $N^{1 /(\mathrm{b}+1)}\left(L_{+}-\mu_{1}\right)$ converges to the cumulative distribution function of the Weibull distribution,

$$
G_{\mathrm{b}+1}(s):=1-\exp \left(-\frac{C_{\mu} s^{\mathrm{b}+1}}{(\mathrm{~b}+1)}\right)
$$

where

$$
C_{\mu}:=\left(\frac{\lambda}{\lambda^{2}-\lambda_{+}^{2}}\right)^{\mathrm{b}+1} \lim _{v \rightarrow 1} \frac{\mu(v)}{(1-v)^{\mathrm{b}}} .
$$

In Section 5 we obtain estimates on the speed of convergence of (2.18); see Corollary 4.9.

Remark 2.9. For $\lambda>\lambda_{+}$, the typical size of the fluctuations of the largest eigenvalues is of order $N^{-1 /(b+1)}$ (with $\mathrm{b}>1$ ) as we can see from Theorem 2.8. For $\lambda<\lambda_{+}$, on the other hand, the fluctuations for the largest eigenvalue become, in the limit $N \rightarrow \infty$, Gaussian with standard deviation of order $N^{-1 / 2}$. (See Appendix C for more detail.)

Remark 2.10. Theorem 2.8 shows that the extreme eigenvalues of $H$ become, for $\lambda>\lambda_{+}$, uncorrelated in the limit $N \rightarrow \infty$. In fact, extending the methods presented in this paper (by choosing $n_{0} \lesssim N^{1 /(\mathrm{b}+1)}$ ), one can show that the point process defined by the (unordered) rescaled extreme eigenvalues of $H$ converges in distribution to an inhomogeneous Poisson point process on $\mathbb{R}^{+}$with intensity function determined by $\lambda$ and $\mu$. 
2.4.2. Eigenvectors behavior. Our second main result asserts that the eigenvectors associated with the largest eigenvalues are "partially localized" for $\lambda>\lambda_{+}$. We denote by $\left(u_{k}(j)\right)_{j=1}^{N}$ the components of the eigenvector $u_{k}$ associated to the eigenvalue $\mu_{k}$. All eigenvectors are normalized as $\sum_{j=1}^{N}\left|u_{k}(j)\right|^{2}=\left\|u_{k}\right\|_{2}^{2}=1$.

Theorem 2.11. Let $W$ be a real symmetric or complex Hermitian Wigner matrix satisfying the assumptions in Definition 2.3. Assume that the distribution $\mu$ is given by (2.10) with $b>1$ and fix some $\lambda>\lambda_{+} ;$see (2.12). Let $n_{0}>10$ be a fixed constant independent of $N$. Then there exist constants $\delta, \delta^{\prime}, \sigma>0$, depending only on $\mathrm{b}, \lambda$ and $\mu$, such that

$$
\mathbb{P}\left(\left.|| u_{k}(k)\right|^{2}-\frac{\lambda^{2}-\lambda_{+}^{2}}{\lambda^{2}} \mid>N^{-\delta}\right) \leq N^{-\sigma}, \quad\left(1 \leq k \leq n_{0}-1\right)
$$

and

$$
\mathbb{P}\left(\left|u_{k}(j)\right|^{2}>\frac{N^{\delta^{\prime}}}{N} \frac{1}{\lambda^{2}\left|v_{k}-v_{j}\right|^{2}}\right) \leq N^{-\sigma}, \quad\left(1 \leq j \leq N, 1 \leq k \leq n_{0}-1, j \neq k\right) .
$$

In Section 7 we obtain explicit expressions for the constants $\delta, \delta^{\prime}, \sigma>0$.

Remark 2.12. In the preceding paper $\left[32\right.$, we proved that all eigenvectors are completely delocalized when $\lambda<\lambda_{+}$. This shows the existence of a sharp transition from the partial localization to the complete delocalization regime. We say that an eigenvalue $\mu_{i}$ is in the bulk of the spectrum of $H$ if $i \in[\epsilon N,(1-\epsilon) N]$, for any (small) $\epsilon>0$ and sufficiently large $N$. Following the proof in [32], can prove that the eigenvectors associated to eigenvalues in the bulk are completely delocalized if $\lambda>\lambda_{+}$.

Assuming that $\mu$ is given by (2.10) with $\mathrm{b} \leq 1$ (and $\mathrm{a} \leq 1$ ), we showed in [32] that all eigenvectors of $H$ are completely delocalized up to the edge.

Remark 2.13. Theorems 2.8 and 2.11 remain valid for deterministic potentials $V$, provided the entires $\left(v_{i}\right)$ satisfy some suitable assumptions; see Definition 4.1 in Section 4 for details.

Remark 2.14. From (2.21) we find

$$
\sum_{j: j \neq k}^{N}\left|u_{k}(j)\right|^{2}=\frac{\lambda_{+}^{2}}{\lambda^{2}}+o(1), \quad\left(1 \leq k \leq n_{0}-1\right)
$$

which is in accordance with the fact that (2.22) holds and that, typically,

$$
\frac{1}{N} \sum_{j: j \neq k}^{N} \frac{1}{\lambda^{2}\left|v_{k}-v_{j}\right|^{2}}=\frac{\lambda_{+}^{2}}{\lambda^{2}}+o(1), \quad\left(1 \leq k \leq n_{0}-1\right),
$$

where we used (2.19).

In the remaining sections, we prove Theorems 2.8 and 2.11. We state our proofs for complex Hermitian matrices. The real symmetric case can be dealt with in the same way.

\section{Preliminaries}

In this section, we collect basic notations and identities.

3.1. Notations. For high probability estimates we use two parameters $\xi \equiv \xi_{N}$ and $\varphi \equiv \varphi_{N}$ : We let

$$
\xi=10 \log \log N, \quad \varphi=(\log N)^{C},
$$

for some fixed constant $C \geq 1$.

Definition 3.1. We say an event $\Omega$ has $(\xi, \nu)$-high probability, if

$$
\mathbb{P}\left(\Omega^{c}\right) \leq \mathrm{e}^{-\nu(\log N)^{\xi}},
$$

for $N$ sufficiently large. Similarly, for a given event $\Omega_{0}$ we say an event $\Omega$ holds with $(\xi, \nu)$-high probability on $\Omega_{0}$, if

$$
\mathbb{P}\left(\Omega_{0} \cap \Omega^{c}\right) \leq \mathrm{e}^{-\nu(\log N)^{\xi}}
$$

for $N$ sufficiently large. 
For brevity, we occasionally say an event holds with high probability, when we mean with $(\xi, \nu)$-high probability. We do not keep track of the explicit value of $\nu$ in the following, allowing $\nu$ to decrease from line to line such that $\nu>0$. From our proof it becomes apparent that such reductions occur only finitely many times.

We define the resolvent, or Green function, $G(z)$, and the averaged Green function, $m(z)$, of $H$ by

$$
G(z)=\left(G_{i j}(z)\right):=\frac{1}{H-z}=\frac{1}{\lambda V+W-z}, \quad m(z):=\frac{1}{N} \operatorname{Tr} G(z), \quad\left(z \in \mathbb{C}^{+}\right)
$$

Frequently, we abbreviate $G \equiv G(z), m \equiv m(z)$, etc. We refer to $z$ as spectral parameter and often write $z=E+$ i $\eta$, $E \in \mathbb{R}, \eta>0$.

We will use double brackets to denote the index set, i.e., for $n_{1}, n_{2} \in \mathbb{R}$,

$$
\llbracket n_{1}, n_{2} \rrbracket:=\left[n_{1}, n_{2}\right] \cap \mathbb{Z} .
$$

We use the symbols $\mathcal{O}(\cdot)$ and $o(\cdot)$ for the standard big-O and little-o notation. The notations $\mathcal{O}, o, \ll, \gg$, refer to the limit $N \rightarrow \infty$ unless otherwise stated, where the notation $a \ll b$ means $a=o(b)$. We use $c$ and $C$ to denote positive constants that do not depend on $N$. Their value may change from line to line. Finally, we write $a \sim b$, if there is $C \geq 1$ such that $C^{-1}|b| \leq|a| \leq C|b|$, and, occasionally, we write for $N$-dependent quantities $a_{N} \lesssim b_{N}$, if there exist constants $C, c>0$ such that $\left|a_{N}\right| \leq C\left(\varphi_{N}\right)^{c \xi}\left|b_{N}\right|$.

3.2. Minors. Let $\mathbb{T} \subset \llbracket 1, N \rrbracket$. Then we define $H^{(\mathbb{T})}$ as the $(N-|\mathbb{T}|) \times(N-|\mathbb{T}|)$ minor of $H$ obtained by removing all columns and rows of $H$ indexed by $i \in \mathbb{T}$. Note that we do not change the names of the indices of $H$ when defining $H^{(\mathbb{T})}$. More specifically, we define an operation $\pi_{i}, i \in \llbracket 1, N \rrbracket$, on the probability space by

$$
\left(\pi_{i}(H)\right)_{k l}:=\mathbb{1}(k \neq i) \mathbb{1}(l \neq i) h_{k l} .
$$

Then, for $\mathbb{T} \subset \llbracket 1, N \rrbracket$, we set $\pi_{\mathbb{T}}:=\prod_{i \in \mathbb{T}} \pi_{i}$ and define

$$
H^{(\mathbb{T})}:=\left(\left(\pi_{\mathbb{T}}(H)_{i j}\right)_{i, j \notin \mathbb{T}}\right.
$$

The Green functions $G^{(\mathbb{T})}$, are defined in an obvious way using $H^{(\mathbb{T})}$. Moreover, we use the shorthand notation

$$
\sum_{i}^{(\mathbb{T})}:=\sum_{\substack{i=1 \\ i \notin \mathbb{T}}}^{N}, \quad \sum_{i \neq j}^{(\mathbb{T})}:=\sum_{\substack{i=1, j=1 \\ i \neq j, i, j \notin \mathbb{T}}}^{N}
$$

abbreviate $(i)=(\{i\}),(\mathbb{T} i)=(\mathbb{T} \cup\{i\})$ and use the convention $(\mathbb{T} \backslash i)=(\mathbb{T} \backslash\{i\})$, if $i \in \mathbb{T},(\mathbb{T} \backslash i)=(\mathbb{T})$, else. In Green function entries $\left(G_{i j}^{(\mathbb{T})}\right)$ we refer to $\{i, j\}$ as lower indices and to $\mathbb{T}$ as upper indices.

Finally, we set

$$
m^{(\mathbb{T})}:=\frac{1}{N} \sum_{i}^{(\mathbb{T})} G_{i i}^{(\mathbb{T})}
$$

Here, we use the normalization $N^{-1}$, instead $(N-|\mathbb{T}|)^{-1}$, since it is more convenient for our computations.

3.3. Resolvent identities. The next lemma collects the main identities between resolvent matrix elements of $H$ and $H^{(\mathbb{T})}$.

Lemma 3.2. Let $H=H^{*}$ be an $N \times N$ matrix. Consider the Green function $G(z) \equiv G:=(H-z)^{-1}, z \in \mathbb{C}^{+}$. Then, for $i, j, k, l \in \llbracket 1, N \rrbracket$, the following identities hold:

- Schur complement/Feshbach formula: For any $i$,

$$
G_{i i}=\frac{1}{h_{i i}-z-\sum_{k, l}^{(i)} h_{i k} G_{k l}^{(i)} h_{l i}} .
$$

- For $i \neq j$,

$$
G_{i j}=-G_{i i} G_{j j}^{(i)}\left(h_{i j}-\sum_{k, l}^{(i j)} h_{i k} G_{k l}^{(i j)} h_{l j}\right)
$$


- For $i \neq j$,

$$
G_{i j}=-G_{i i} \sum_{k}^{(i)} h_{i k} G_{k j}^{(i)}=-G_{j j} \sum_{k}^{(j)} G_{i k}^{(j)} h_{k j} .
$$

- For $i, j \neq k$,

$$
G_{i j}=G_{i j}^{(k)}+\frac{G_{i k} G_{k j}}{G_{k k}}
$$

- Ward identity: For any $i$,

$$
\sum_{j=1}^{N}\left|G_{i j}\right|^{2}=\frac{1}{\eta} \operatorname{Im} G_{i i}
$$

where $\eta=\operatorname{Im} z$.

For a proof we refer to, e.g., [14].

Lemma 3.3. There is a constant $C$ such that, for any $z \in \mathbb{C}^{+}, i \in \llbracket 1, N \rrbracket$, we have

$$
\left|m(z)-m^{(i)}(z)\right| \leq \frac{C}{N \eta} .
$$

The lemma follows from Cauchy's interlacing property of eigenvalues of $H$ and its minor $H^{(i)}$. For a detailed proof we refer to $\llbracket 12$. For $\mathbb{T} \subset \llbracket 1, N \rrbracket$, with, say, $|\mathbb{T}| \leq 10$, we obtain $\left|m-m^{(\mathbb{T})}\right| \leq \frac{C}{N \eta}$.

3.4. Large deviation estimates. We collect here some useful large deviation estimates for random variables with slowly decaying moments.

Lemma 3.4. Let $\left(a_{i}\right)$ and $\left(b_{i}\right)$ be centered and independent complex random variables with variance $\sigma^{2}$ and having subexponential decay

$$
\mathbb{P}\left(\left|a_{i}\right| \geq x \sigma\right) \leq C_{0} \mathrm{e}^{-x^{1 / \theta}}, \quad \mathbb{P}\left(\left|b_{i}\right| \geq x \sigma\right) \leq C_{0} \mathrm{e}^{-x^{1 / \theta}},
$$

for some positive constants $C_{0}$ and $\theta>1$. For $i, j \in \llbracket 1, N \rrbracket$, let $A_{i} \in \mathbb{C}$ and $B_{i j} \in \mathbb{C}$. Then there exists a constant $c_{0}$, depending only on $\theta$ and $C_{0}$, such that for $1<\xi \leq 10 \log \log N$ and $\varphi_{N}=(\log N)^{c_{0}}$ the following estimates hold.

(1)

$$
\begin{array}{r}
\mathbb{P}\left(\left|\sum_{i=1}^{N} A_{i} a_{i}\right| \geq\left(\varphi_{N}\right)^{\xi} \sigma\left(\sum_{i=1}^{N}\left|A_{i}\right|^{2}\right)^{1 / 2}\right) \leq \mathrm{e}^{-(\log N)^{\xi}}, \\
\mathbb{P}\left(\left|\sum_{i=1}^{N} \bar{a}_{i} B_{i i} a_{i}-\sum_{i=1}^{N} \sigma^{2} B_{i i}\right| \geq\left(\varphi_{N}\right)^{\xi} \sigma^{2}\left(\sum_{i=1}^{N}\left|B_{i i}\right|^{2}\right)^{1 / 2}\right) \leq \mathrm{e}^{-(\log N)^{\xi}}, \\
\mathbb{P}\left(\left|\sum_{i \neq j}^{N} \bar{a}_{i} B_{i j} a_{j}\right| \geq\left(\varphi_{N}\right)^{2 \xi} \sigma^{2}\left(\sum_{i \neq j}\left|B_{i j}\right|^{2}\right)^{1 / 2}\right) \leq \mathrm{e}^{-(\log N)^{\xi}},
\end{array}
$$

for $N$ sufficiently large;

(2)

$$
\mathbb{P}\left(\left|\sum_{i, j} \bar{a}_{i} B_{i j} b_{j}\right| \geq\left(\varphi_{N}\right)^{2 \xi} \sigma^{2}\left(\sum_{i, j}\left|B_{i j}\right|^{2}\right)^{1 / 2}\right) \leq \mathrm{e}^{-(\log N)^{\xi}},
$$

for $N$ sufficiently large.

For a proof we refer to [23. We now choose $C$ in (3.1) such that $C \geq c_{0}$.

Finally, we point out the difference between the random variables $\left(w_{i j}\right)$ and $\left(v_{i}\right)$ : From (2.5), we obtain

$$
\left|w_{i j}\right| \leq \frac{\left(\varphi_{N}\right)^{\xi}}{\sqrt{N}},
$$

with $(\xi, \nu)$-high probability, whereas $v_{i} \in[-1,1]$, almost surely. 


\section{Proof of Theorem 2.8}

In this section, we outline the proof of Theorem 2.8. We first fix the diagonal random entries $\left(v_{i}\right)$ and consider $\widehat{\mu}_{f c}$, the deformed semicircle measure with fixed $\left(v_{i}\right)$. The main tools we use in the proof are Lemma 4.2, where we obtain a linear approximation of $m_{f c}$, and Lemma 4.5. which estimates the difference between $m_{f c}$ and $\widehat{m}_{f c}$, the latter being the Stieltjes transform of $\widehat{\mu}_{f c}$. Using Proposition 4.6 that estimates the eigenvalue locations in terms of $\widehat{m}_{f c}$, we prove Theorem 2.8 .

4.1. Definition of $\Omega_{V}$. In this subsection we define an event $\Omega_{V}$, on which the random variables $\left(v_{i}\right)$ exhibit "typical" behavior. For this purpose we need some more notation: Denote by $\mathfrak{b}$ the constant

$$
\mathfrak{b}:=\frac{1}{2}-\frac{1}{\mathrm{~b}+1}=\frac{\mathrm{b}-1}{2(\mathrm{~b}+1)}=\frac{\mathrm{b}}{\mathrm{b}+1}-\frac{1}{2}
$$

which only depends on b. Fix some small $\epsilon>0$ satisfying

$$
\epsilon<\left(10+\frac{\mathrm{b}+1}{\mathrm{~b}-1}\right) \mathfrak{b},
$$

and define the domain, $\mathcal{D}_{\epsilon}$, of the spectral parameter $z$ by

$$
\mathcal{D}_{\epsilon}:=\left\{z=E+\mathrm{i} \eta \in \mathbb{C}^{+}:-3-\lambda \leq E \leq 3+\lambda, N^{-1 / 2-\epsilon} \leq \eta \leq N^{-1 /(\mathrm{b}+1)+\epsilon}\right\} .
$$

Using spectral perturbation theory, we find that the following a priori bound

$$
\left|\mu_{k}\right| \leq\|H\| \leq\|W\|+\lambda\|V\| \leq 2+\lambda+\left(\varphi_{N}\right)^{c \xi} N^{-2 / 3}, \quad(k \in \llbracket 1, N \rrbracket),
$$

holds with high probability; see, e.g., Theorem 2.1. in 25].

Further, we define $N$-dependent constants $\kappa_{0}$ and $\eta_{0}$ by

$$
\kappa_{0}:=N^{-1 /(\mathrm{b}+1)}, \quad \eta_{0}:=\frac{N^{-\epsilon}}{\sqrt{N}} .
$$

In the following, typical choices for $z \equiv L_{+}-\kappa+$ i $\eta$ will be such that $\kappa$ and $\eta$ satisfy $\kappa \lesssim \kappa_{0}$ and $\eta \geq \eta_{0}$.

We are now prepared to give a definition of the "good" event $\Omega_{V}$ :

Definition 4.1. Let $n_{0}>10$ be a fixed positive integer independent of $N$. We define $\Omega_{V}$ to be the event on which the following conditions hold for any $k \in \llbracket 1, n_{0}-1 \rrbracket$ :

1. The $k$-th largest random variable $v_{k}$ satisfies, for all $j \in \llbracket 1, N \rrbracket$ with $j \neq k$,

$$
N^{-\epsilon} \kappa_{0}<\left|v_{j}-v_{k}\right|<(\log N) \kappa_{0} .
$$

In addition, for $k=1$, we have

$$
N^{-\epsilon} \kappa_{0}<\left|1-v_{1}\right|<(\log N) \kappa_{0} .
$$

2. There exists a constant $\mathfrak{c}<1$ independent of $N$ such that, for any $z \in \mathcal{D}_{\epsilon}$ satisfying

$$
\min _{i \in \llbracket 1, N \rrbracket}\left|\operatorname{Re}\left(z+m_{f c}(z)\right)-\lambda v_{i}\right|=\left|\operatorname{Re}\left(z+m_{f c}(z)\right)-\lambda v_{k}\right|,
$$

we have

$$
\frac{1}{N} \sum_{i}^{(k)} \frac{1}{\left|\lambda v_{i}-z-m_{f c}(z)\right|^{2}}<\mathfrak{c}<1 .
$$

We remark that, together with (4.6) and (4.7), (4.8) implies

$$
\left|\operatorname{Re}\left(z+m_{f c}(z)\right)-\lambda v_{i}\right|>\frac{N^{-\epsilon} \kappa_{0}}{2},
$$

for all $i \neq k$.

3. There exists a constant $C>0$ such that, for any $z \in \mathcal{D}_{\epsilon}$, we have

$$
\left|\frac{1}{N} \sum_{i=1}^{N} \frac{1}{\lambda v_{i}-z-m_{f c}(z)}-\int \frac{\mathrm{d} \mu(v)}{\lambda v-z-m_{f c}(z)}\right| \leq \frac{C N^{3 \epsilon / 2}}{\sqrt{N}} .
$$

In Appendix A we show that

$$
\mathbb{P}\left(\Omega_{V}\right) \geq 1-C(\log N)^{1+2 \mathrm{~b}} N^{-\epsilon}
$$

thus $\left(\Omega_{V}\right)^{c}$ is indeed a rare event. 
4.2. Definition of $\widehat{m}_{f c}$. Let $\widehat{\mu}$ be the empirical measure defined by

$$
\widehat{\mu}:=\frac{1}{N} \sum_{i=1}^{N} \delta_{\lambda v_{i}}
$$

We define a random measure $\widehat{\mu}_{f c}$ by setting $\widehat{\mu}_{f c}:=\widehat{\mu} \boxplus \mu_{s c}$, i.e., $\widehat{\mu}_{f c}$ is the additive free convolution of the empirical measure $\widehat{\mu}$ and the semicircular measure $\mu_{s c}$. As in the case of $m_{f c}$, the Stieltjes transform $\widehat{m}_{f c}$ of the measure $\widehat{\mu}_{f c}$ is a solution to the equation

$$
\widehat{m}_{f c}(z)=\frac{1}{N} \sum_{i=1}^{N} \frac{1}{\lambda v_{i}-z-\widehat{m}_{f c}(z)}, \quad \operatorname{Im} \widehat{m}_{f c}(z) \geq 0, \quad\left(z \in \mathbb{C}^{+}\right),
$$

and we obtain $\widehat{\mu}_{f c}$ trough the Stieltjes inversion formula from $\widehat{m}_{f c}(z)$, c.f., (2.3).

Recall that we assume that $v_{1}>v_{2}>\ldots>v_{N}$. Assuming that $\Omega_{V}$ holds, i.e., $\left(v_{i}\right)$ are fixed and satisfy the conditions in Definition 4.1, we are going to show that $m_{f c}(z)$ is a good approximation of $\widehat{m}_{f c}(z)$ for $z$ in some subset of $\mathcal{D}_{\epsilon}$.

4.3. Properties of $m_{f c}$ and $\widehat{m}_{f c}$. Recall the definitions of $m_{f c}$ and $\widehat{m}_{f c}$. Let

$$
R_{2}(z):=\int \frac{\mathrm{d} \mu(v)}{\left|\lambda v-z-m_{f c}(z)\right|^{2}}, \quad \widehat{R}_{2}(z):=\frac{1}{N} \sum_{i=1}^{N} \frac{1}{\left|\lambda v_{i}-z-\widehat{m}_{f c}(z)\right|^{2}}, \quad\left(z \in \mathbb{C}^{+}\right) .
$$

Since

we have that

$$
\operatorname{Im} m_{f c}(z)=\int \frac{\operatorname{Im} z+\operatorname{Im} m_{f c}(z)}{\left|\lambda v-z-m_{f c}(z)\right|^{2}} \mathrm{~d} \mu(v)
$$

$$
R_{2}(z)=\frac{\operatorname{Im} m_{f c}(z)}{\operatorname{Im} z+\operatorname{Im} m_{f c}(z)}<1, \quad\left(z \in \mathbb{C}^{+}\right) .
$$

Similarly, we also find that $\widehat{R}_{2}(z)<1$.

The following lemma shows that $m_{f c}$ is approximately a linear function near the spectral edge.

Lemma 4.2. Let $z=L_{+}-\kappa+\mathrm{i} \eta \in \mathcal{D}_{\epsilon}$. Then,

$$
z+m_{f c}(z)=\lambda-\frac{\lambda^{2}}{\lambda^{2}-\lambda_{+}^{2}}\left(L_{+}-z\right)+\mathcal{O}\left((\log N)(\kappa+\eta)^{\min \{\mathrm{b}, 2\}}\right) .
$$

Similarly, if $z, z^{\prime} \in \mathcal{D}_{\epsilon}$, then

$$
m_{f c}(z)-m_{f c}\left(z^{\prime}\right)=\frac{\lambda_{+}^{2}}{\lambda^{2}-\lambda_{+}^{2}}\left(z-z^{\prime}\right)+\mathcal{O}\left((\log N)^{2}\left(N^{-1 /(\mathrm{b}+1)}\right)^{\min \{\mathrm{b}-1,1\}}\left|z-z^{\prime}\right|\right) .
$$

Proof. We only prove the first part of the lemma; the second part is proved analogously. Since $L_{+}+m_{f c}\left(L_{+}\right)=\lambda$, see Lemma 2.4. we can write

$$
\begin{aligned}
m_{f c}(z)-m_{f c}\left(L_{+}\right) & =\int \frac{\mathrm{d} \mu(v)}{\lambda v-z-m_{f c}(z)}-\int \frac{\mathrm{d} \mu(v)}{\lambda v-L_{+}-m_{f c}\left(L_{+}\right)} \\
& =\int \frac{m_{f c}(z)-m_{f c}\left(L_{+}\right)+\left(z-L_{+}\right)}{\left(\lambda v-z-m_{f c}(z)\right)(\lambda v-\lambda)} \mathrm{d} \mu(v)
\end{aligned}
$$

Setting

$$
T(z):=\int \frac{\mathrm{d} \mu(v)}{\left(\lambda v-z-m_{f c}(z)\right)(\lambda v-\lambda)}
$$

we find

$$
|T(z)| \leq\left(\int \frac{\mathrm{d} \mu(v)}{\left|\lambda v-z-m_{f c}(z)\right|^{2}}\right)^{1 / 2}\left(\int \frac{\mathrm{d} \mu(v)}{|\lambda v-\lambda|^{2}}\right)^{1 / 2} \leq \sqrt{R_{2}(z)} \frac{\lambda_{+}}{\lambda}<\frac{\lambda_{+}}{\lambda}<1 .
$$

Hence, for $z \in \mathcal{D}_{\epsilon}$, we have

$$
m_{f c}(z)-m_{f c}\left(L_{+}\right)=\frac{T(z)}{1-T(z)}\left(z-L_{+}\right)
$$


which shows that

$$
z+m_{f c}(z)=\lambda-\frac{1}{1-T(z)}\left(L_{+}-z\right) .
$$

We thus obtain from (4.20) and (4.22) that

$$
\left|z+m_{f c}(z)-\lambda\right| \leq \frac{\lambda}{\lambda-\lambda_{+}}\left|L_{+}-z\right| .
$$

We now estimate the difference $T(z)-\lambda_{+}^{2} / \lambda^{2}:$ Let $\tau:=z+m_{f c}(z)$. We have

$$
T(z)-\frac{\lambda_{+}^{2}}{\lambda^{2}}=\int \frac{\mathrm{d} \mu(v)}{(\lambda v-\tau)(\lambda v-\lambda)}-\int \frac{\mathrm{d} \mu(v)}{(\lambda v-\lambda)^{2}}=(\tau-\lambda) \int \frac{\mathrm{d} \mu(v)}{(\lambda v-\tau)(\lambda v-\lambda)^{2}} .
$$

In order to find an upper bound on the integral on the very right side, we consider the following cases:

(1) When $\mathrm{b} \geq 2$, we have

$$
\left|\int \frac{\mathrm{d} \mu(v)}{(\lambda v-\tau)(\lambda v-\lambda)^{2}}\right| \leq C \int_{-1}^{1} \frac{\mathrm{d} v}{|\lambda v-\tau|} \leq C \log N .
$$

(2) When $\mathrm{b}<2$, define a set $B \subset[-1,1]$ by

$$
B:=\{v \in[-1,1]: \lambda v<-\lambda+2 \operatorname{Re} \tau\},
$$

and $B^{c} \equiv[-1,1] \backslash B$. Estimating the integral in (4.23) on $B$ we find

$$
\left|\int_{B} \frac{\mathrm{d} \mu(v)}{(\lambda v-\tau)(\lambda v-\lambda)^{2}}\right| \leq C \int_{B} \frac{\mathrm{d} \mu(v)}{|\lambda v-\lambda|^{3}} \leq C|\lambda-\tau|^{\mathrm{b}-2},
$$

where we have used that, for $v \in B$,

$$
|\lambda v-\tau|>|\operatorname{Re} \tau-\lambda v|>\frac{1}{2}(\lambda-\lambda v) .
$$

On the set $B^{c}$, we have

$$
\left|\int_{B^{c}} \frac{\mathrm{d} \mu(v)}{(\lambda v-\tau)(\lambda v-\lambda)}\right| \leq C \int_{B^{c}} \frac{|\lambda-\lambda v|^{\mathrm{b}-1}}{|\lambda v-\tau|} \mathrm{d} v \leq C|\lambda-\tau|^{\mathrm{b}-1} \log N
$$

where we have used that, for $v \in B^{c}$,

$$
|\lambda-\lambda v| \leq 2(\lambda-\operatorname{Re} \tau) \leq 2|\lambda-\tau|
$$

We also have

$$
\left|\int_{B^{c}} \frac{\mathrm{d} \mu(v)}{(\lambda v-\lambda)^{2}}\right| \leq C \int_{B^{c}}|\lambda v-\lambda|^{\mathrm{b}-2} \mathrm{~d} v \leq C|\lambda-\tau|^{\mathrm{b}-1} .
$$

Thus, we obtain from (4.23), (4.26) and (4.27) that

$$
\left|\int \frac{\mathrm{d} \mu(v)}{(\lambda v-\tau)(\lambda v-\lambda)^{2}}\right| \leq C|\lambda-\tau|^{\mathrm{b}-2} \log N .
$$

Since $T(z)$ is continuous and $\mathcal{D}_{\epsilon}$ is compact, we can choose the constants uniform in $z$. We thus have proved that

$$
T(z)=\frac{\lambda_{+}^{2}}{\lambda^{2}}+\mathcal{O}\left((\log N)\left|L_{+}-z\right|^{\min \{b-1,1\}}\right),
$$

which, combined with (4.22), proves the desired lemma.

Remark 4.3. Choosing in Lemma $4.2 z=z_{k}$, where $z_{k}:=L_{+}-\kappa_{k}+\mathrm{i} \eta \in \mathcal{D}_{\epsilon}$ with

$$
\kappa_{k}=\frac{\lambda^{2}-\lambda_{+}^{2}}{\lambda}\left(1-v_{k}\right)
$$

we obtain

$$
z_{k}+m_{f c}\left(z_{k}\right)=\lambda v_{k}+\frac{\lambda^{2}}{\lambda^{2}-\lambda_{+}^{2}} \eta+\mathcal{O}\left((\log N) N^{-\min \{\mathrm{b}, 2\} /(\mathrm{b}+1)+2 \epsilon}\right)
$$


To estimate the difference $\left|\widehat{m}_{f c}-m_{f c}\right|$, we consider the following subset of $\mathcal{D}_{\epsilon}$.

Definition 4.4. Let $A:=\llbracket n_{0}, N \rrbracket$. We define the domain $\mathcal{D}_{\epsilon}^{\prime}$ of the spectral parameter $z$ as

$$
\mathcal{D}_{\epsilon}^{\prime}=\left\{z \in \mathcal{D}_{\epsilon}:\left|\lambda v_{a}-z-m_{f c}(z)\right|>\frac{1}{2} N^{-1 /(\mathrm{b}+1)-\epsilon}, \forall a \in A\right\} .
$$

Eventually, we are going to show that $\mu_{k}+\mathrm{i} \eta_{0} \in \mathcal{D}_{\epsilon}^{\prime}, k \in \llbracket 1, n_{0}-1 \rrbracket$, with high probability on $\Omega_{V}$; see Remark 4.7 . We now prove an a priori bound on the difference $\left|\widehat{m}_{f c}-m_{f c}\right|$ on $\mathcal{D}_{\epsilon}^{\prime}$.

Lemma 4.5. For any $z \in \mathcal{D}_{\epsilon}^{\prime}$, we have on $\Omega_{V}$ that

$$
\left|\widehat{m}_{f c}(z)-m_{f c}(z)\right| \leq \frac{N^{2 \epsilon}}{\sqrt{N}} .
$$

Proof. Assume that $\Omega_{V}$ holds. For given $z \in \mathcal{D}_{\epsilon}^{\prime}$, choose $k \in \llbracket 1, n_{0}-1 \rrbracket$ satisfying (4.8), i.e., among $\left(\lambda v_{i}\right), \lambda v_{k}$ is closest to $\operatorname{Re}\left(z+m_{f c}(z)\right)$. Suppose that (4.32) does not hold. Using the definitions of $m_{f c}$ and $\widehat{m}_{f c}$, we obtain the following self-consistent equation for $\left(\widehat{m}_{f c}-m_{f c}\right)$ :

$$
\begin{aligned}
& \widehat{m}_{f c}-m_{f c}=\frac{1}{N} \sum_{i=1}^{N}\left(\frac{1}{\lambda v_{i}-z-\widehat{m}_{f c}}-m_{f c}\right) \\
& =\frac{1}{N} \sum_{i=1}^{N}\left(\frac{1}{\lambda v_{i}-z-\widehat{m}_{f c}}-\frac{1}{\lambda v_{i}-z-m_{f c}}\right)+\left(\frac{1}{N} \sum_{i=1}^{N} \frac{1}{\lambda v_{i}-z-m_{f c}}-\int \frac{\mathrm{d} \mu(v)}{\lambda v-z-m_{f c}}\right) \\
& =\frac{1}{N} \sum_{i=1}^{N} \frac{\widehat{m}_{f c}-m_{f c}}{\left(\lambda v_{i}-z-\widehat{m}_{f c}\right)\left(\lambda v_{i}-z-m_{f c}\right)}+\left(\frac{1}{N} \sum_{i=1}^{N} \frac{1}{\lambda v_{i}-z-m_{f c}}-\int \frac{\mathrm{d} \mu(v)}{\lambda v-z-m_{f c}}\right) .
\end{aligned}
$$

From the assumption (4.11), we find that the second term in the right hand side of (4.33) is bounded by $N^{-1 / 2+3 \epsilon / 2}$.

Next, we estimate the first term in the right hand side of (4.33). For $i=k$, we have

$$
\left|\lambda v_{k}-z-\widehat{m}_{f c}\right|+\left|\lambda v_{k}-z-m_{f c}\right| \geq\left|\widehat{m}_{f c}(z)-m_{f c}(z)\right|>\frac{N^{2 \epsilon}}{\sqrt{N}}
$$

which shows that either

$$
\left|\lambda v_{k}-z-\widehat{m}_{f c}\right| \geq \frac{N^{2 \epsilon}}{2 \sqrt{N}} \quad \text { or } \quad\left|\lambda v_{k}-z-m_{f c}\right| \geq \frac{N^{2 \epsilon}}{2 \sqrt{N}} .
$$

In either case, by considering the imaginary part, we find

$$
\frac{1}{N}\left|\frac{1}{\left(\lambda v_{k}-z-\widehat{m}_{f c}\right)\left(\lambda v_{k}-z-m_{f c}\right)}\right| \leq \frac{1}{N} \frac{2 \sqrt{N}}{N^{2 \epsilon}} \frac{1}{\eta} \leq C N^{-\epsilon}, \quad\left(z \in \mathcal{D}_{\epsilon}^{\prime}\right) .
$$

For the other terms, we use

$$
\frac{1}{N}\left|\sum_{i}^{(k)} \frac{1}{\left(\lambda v_{i}-z-\widehat{m}_{f c}\right)\left(\lambda v_{i}-z-m_{f c}\right)}\right| \leq \frac{1}{2 N} \sum_{i}^{(k)}\left(\frac{1}{\left|\lambda v_{i}-z-\widehat{m}_{f c}\right|^{2}}+\frac{1}{\left|\lambda v_{i}-z-m_{f c}\right|^{2}}\right) .
$$

From (4.14), we have that

$$
\frac{1}{N} \sum_{i=1}^{N} \frac{1}{\left|\lambda v_{i}-z-\widehat{m}_{f c}\right|^{2}}=\frac{\operatorname{Im} \widehat{m}_{f c}}{\eta+\operatorname{Im} \widehat{m}_{f c}}<1 .
$$

We also assume in the assumption (4.9) that

$$
\frac{1}{N} \sum_{i}^{(k)} \frac{1}{\left|\lambda v_{i}-z-m_{f c}\right|^{2}}<\mathfrak{c}<1,
$$

for some constant $c$. Thus, we get

$$
\left|\widehat{m}_{f c}(z)-m_{f c}(z)\right|<\frac{1+\mathfrak{c}}{2}\left|\widehat{m}_{f c}(z)-m_{f c}(z)\right|+N^{-1 / 2+3 \epsilon / 2}, \quad\left(z \in \mathcal{D}_{\epsilon}^{\prime}\right),
$$

which implies that

$$
\left|\widehat{m}_{f c}(z)-m_{f c}(z)\right|<C N^{-1 / 2+3 \epsilon / 2}, \quad\left(z \in \mathcal{D}_{\epsilon}^{\prime}\right) .
$$

Since this contradicts the assumption that (4.32) does not hold, it proves the desired lemma. 
4.4. Proof of Theorem 2.8. The main result of this subsection is Proposition 4.8, which will imply Theorem 2.8, The key ingredient of the proof of Proposition 4.8 is an implicit equation for the largest eigenvalues $\left(\mu_{k}\right)$ of $H$. This equation, Equation (4.38) in Proposition 4.6 below, involves the Stieltjes transform $\widehat{m}_{f c}$ and the random variables $\left(v_{k}\right)$. Using the information on $\widehat{m}_{f c}$ gathered in the previous subsections, we can solve Equation (4.38) approximately for $\left(\mu_{k}\right)$. The proof of Proposition 4.6 is postponed to Section 5.

Proposition 4.6. Let $n_{0}>10$ be a fixed integer independent of $N$. Let $\mu_{k}$ be the $k$-th largest eigenvalue of $H$, $k \in \llbracket 1, n_{0}-1 \rrbracket$. Suppose that the assumptions in Theorem 2.8 hold. Then, the following holds with $(\xi-2, \nu)$-high probability on $\Omega_{V}$ :

$$
\mu_{k}+\operatorname{Re} \widehat{m}_{f c}\left(\mu_{k}+\mathrm{i} \eta_{0}\right)=\lambda v_{k}+\mathcal{O}\left(N^{-1 / 2+3 \epsilon}\right)
$$

where $\eta_{0}$ is defined in (4.5).

Remark 4.7. Since $\left|\lambda v_{i}-\lambda v_{k}\right| \geq N^{-\epsilon} \kappa_{0} \gg N^{-1 / 2+3 \epsilon}$, for all $i \neq k$, on $\Omega_{V}$, we obtain from Proposition 4.6 that

$$
\left|\mu_{k}+\mathrm{i} \eta_{0}+\operatorname{Re} \widehat{m}_{f c}\left(\mu_{k}+\mathrm{i} \eta_{0}\right)-\lambda v_{i}\right| \geq\left|\lambda v_{i}-\lambda v_{k}\right|-\left|\mu_{k}+\mathrm{i} \eta_{0}+\operatorname{Re} \widehat{m}_{f c}\left(\mu_{k}+\mathrm{i} \eta_{0}\right)-\lambda v_{k}\right| \geq \frac{N^{-\epsilon} \kappa_{0}}{2}
$$

on $\Omega_{V}$. Hence, we find that $\mu_{k}+\mathrm{i} \eta_{0} \in \mathcal{D}_{\epsilon}^{\prime}, k \in \llbracket 1, n_{0}-1 \rrbracket$, with high probability on $\Omega_{V}$.

Combining the tools developed in the previous subsection, we now prove the main result on the eigenvalue locations.

Proposition 4.8. Let $n_{0}>10$ be a fixed integer independent of $N$. Let $\mu_{k}$ be the $k$-th largest eigenvalue of $H=\lambda V+W$, where $k \in \llbracket 1, n_{0}-1 \rrbracket$. Then, there exist constants $C$ and $\nu>0$ such that we have

$$
\left|\mu_{k}-\left(L_{+}-\frac{\lambda^{2}-\lambda_{+}^{2}}{\lambda}\left(1-v_{k}\right)\right)\right| \leq C \frac{1}{N^{1 /(\mathrm{b}+1)}}\left(\frac{N^{3 \epsilon}}{N^{\mathfrak{b}}}+\frac{(\log N)^{2}}{N^{1 /(\mathrm{b}+1)}}\right),
$$

with $(\xi-2, \nu)$-high probability on $\Omega_{V}$.

Proof of Theorem [2.8 and Proposition 4.8. It suffices to prove Proposition4.8, Let $k \in \llbracket 1, n_{0}-1 \rrbracket$. From Lemma4.5 and Proposition 4.6, we find that, with high probability on $\Omega_{V}$,

$$
\mu_{k}+\operatorname{Re} m_{f c}\left(\mu_{k}+\mathrm{i} \eta_{0}\right)=\lambda v_{k}+\mathcal{O}\left(N^{-1 / 2+3 \epsilon}\right) .
$$

In Lemma 4.2, we showed that

$$
\mu_{k}+\mathrm{i} \eta_{0}+m_{f c}\left(\mu_{k}+\mathrm{i} \eta_{0}\right)=\lambda-\frac{\lambda^{2}}{\lambda^{2}-\lambda_{+}^{2}}\left(L_{+}-\mu_{k}\right)+\mathrm{i} C \eta_{0}+\mathcal{O}\left(\kappa_{0}^{\min \{\mathrm{b}, 2\}}(\log N)^{2}\right) .
$$

Thus, we obtain

$$
\mu_{k}+\operatorname{Re} m_{f c}\left(\mu_{k}+\mathrm{i} \eta_{0}\right)=\lambda-\frac{\lambda^{2}}{\lambda^{2}-\lambda_{+}^{2}}\left(L_{+}-\mu_{k}\right)+\mathcal{O}\left(\kappa_{0}^{\min \{\mathrm{b}, 2\}}(\log N)^{2}\right) .
$$

Therefore, we have with high probability on $\Omega_{V}$ that

$$
\mu_{k}=L_{+}-\frac{\lambda^{2}-\lambda_{+}^{2}}{\lambda}\left(1-v_{k}\right)+\mathcal{O}\left(\kappa_{0}^{\min \{\mathrm{b}, 2\}}(\log N)^{2}\right)+\mathcal{O}\left(N^{-1 / 2+3 \epsilon}\right),
$$

completing the proof of Proposition 4.8.

Recalling that $\mathbb{P}\left(\Omega_{V}\right) \geq 1-C(\log N)^{1+2 \mathrm{~b}} N^{-\epsilon}$, we obtain from Proposition 4.8 the following corollary.

Corollary 4.9. Let $n_{0}$ be a fixed constant independent of $N$. Let $\mu_{k}$ be the $k$-th largest eigenvalue of $H=\lambda V+W$, where $1 \leq k<n_{0}$. Then, there exists a constant $C_{1}>0$ such that for $s \in \mathbb{R}^{+}$we have

$$
\begin{aligned}
\mathbb{P} & \left(N^{1 /(\mathrm{b}+1)} \frac{\lambda^{2}-\lambda_{+}^{2}}{\lambda}\left(1-v_{k}\right) \leq s-C_{1}\left(\frac{N^{3 \epsilon}}{N^{\mathfrak{b}}}+\frac{(\log N)^{2}}{N^{1 /(\mathrm{b}+1)}}\right)\right)-C_{1} \frac{(\log N)^{1+2 \mathrm{~b}}}{N^{\epsilon}} \\
& \leq \mathbb{P}\left(N^{1 /(\mathrm{b}+1)}\left(L_{+}-\mu_{k}\right) \leq s\right) \\
& \leq \mathbb{P}\left(N^{1 /(\mathrm{b}+1)} \frac{\lambda^{2}-\lambda_{+}^{2}}{\lambda}\left(1-v_{k}\right) \leq s+C_{1}\left(\frac{N^{3 \epsilon}}{N^{\mathfrak{b}}}+\frac{(\log N)^{2}}{N^{1 /(\mathrm{b}+1)}}\right)\right)+C_{1} \frac{(\log N)^{1+2 \mathrm{~b}}}{N^{\epsilon}},
\end{aligned}
$$

for $N$ sufficiently large.

Remark 4.10. The constants in Proposition 4.8 and Corollary 4.9 depend only on $\lambda$, the distribution $\mu$ and the constants $C_{0}$ and $\theta$ in (2.5), but are otherwise independent of the detailed structure of the Wigner matrix $W$. 


\section{Estimates on the Location of the Eigenvalues}

In this section, we prove Proposition 4.6. Recall the definition of $\eta_{0}$ in (4.5). For $k \in \llbracket 1, n_{0}-1 \rrbracket$, let $\widehat{E}_{k} \in \mathbb{R}$ be a solution $E=\widehat{E}_{k}$ to the equation

$$
E+\operatorname{Re} \widehat{m}_{f c}\left(E+\mathrm{i} \eta_{0}\right)=\lambda v_{k}, \quad(E \in \mathbb{R}),
$$

and set $\widehat{z}_{k}:=\widehat{E}_{k}+\mathrm{i} \eta_{0}$. The existence of such $\widehat{E}_{k}$ is easy to see from Lemma 4.2 and Lemma 4.5, If there are two or more solutions to (5.1), we choose $\widehat{E}_{k}$ to be the largest one among these solutions. The key observation used in the proof of Proposition 4.6 is that $\operatorname{Im} m(z)$, the imaginary part of the averaged Green function, has a sharp peak if and only if the imaginary part of

$$
g_{k}(z):=\frac{1}{\lambda v_{k}-z-\widehat{m}_{f c}(z)}, \quad\left(z \in \mathbb{C}^{+}\right),
$$

becomes sufficiently large for some $k \in \llbracket 1, n_{0}-1 \rrbracket$. Since $z \mapsto \operatorname{Im} g_{k}(z)$ has a sharp peak near $\widehat{z}_{k}$, we can then conclude that $z \mapsto \operatorname{Im} m(z)$ also has a peak near $\widehat{z}_{k}$. From the spectral decomposition

$$
\operatorname{Im} m\left(E+\mathrm{i} \eta_{0}\right)=\frac{1}{N} \sum_{\alpha=1}^{N} \frac{\eta_{0}}{\left(\mu_{\alpha}-E\right)^{2}+\eta_{0}^{2}},
$$

we also observe that the positions of the peaks of $\operatorname{Im} m(z)$ correspond to the locations of the eigenvalues. This will enable us to estimate the location of the $k$-th largest eigenvalue in terms of $v_{k}$, yielding a proof of Proposition 4.6 ,

This section is organized as follows. In Subsection 5.1, we establish a local law for $m(z)$ with $z \in \mathcal{D}_{\epsilon}^{\prime}$, i.e., for $z$ close to the upper edge; see Proposition 5.1 below. In the Subsections 5.2 and 5.3 we establish further estimates that will be used in the proof of Proposition 4.6. The estimates in Subsection 5.3 are rather straightforward, while the estimates of Subsection 5.3 rely on the "fluctuation average lemma" whose proof is postponed to Section 6 . The proof of Proposition 4.6 is then completed in Subsection 5.4.

5.1. Properties of $\widehat{m}_{f c}$ and $m$. In the proof of Proposition 4.6, we will use the following local law as an a priori estimate. Recall the constant $\epsilon>0$ in (4.2) and the definition of the domain $\mathcal{D}_{\epsilon}^{\prime}$ in (4.31).

Proposition 5.1. [Local law near the edge] We have with $(\xi, \nu)$-high probability on $\Omega_{V}$ that

$$
\left|m(z)-\widehat{m}_{f c}(z)\right| \leq \frac{N^{2 \epsilon}}{\sqrt{N}}
$$

for all $z \in \mathcal{D}_{\epsilon}^{\prime}$.

The proof of Proposition 5.1 is the content of the rest of this subsection.

Recall the definitions of $\left(\widehat{z}_{k}\right)$ in (5.1). We begin by deriving a basic property of $\widehat{m}_{f c}(z)$ near $\left(\widehat{z}_{k}\right)$. Recall the definition of $\eta_{0}$ in (4.5).

Lemma 5.2. For $z=E+\mathrm{i} \eta_{0} \in \mathcal{D}_{\epsilon}^{\prime}$, the following hold on $\Omega_{V}$ :

(1) if $\left|z-\widehat{z}_{j}\right| \geq N^{-1 / 2+3 \epsilon}$ for all $j \in \llbracket 1, n_{0}-1 \rrbracket$, then there exists a constant $C>1$ such that

$$
C^{-1} \eta_{0} \leq \operatorname{Im} \widehat{m}_{f c}(z) \leq C \eta_{0} ;
$$

(2) if $z=\widehat{z}_{k}$ for some $k \in \llbracket 1, n_{0}-1 \rrbracket$, then there exists a constant $C>1$ such that

$$
C^{-1} N^{-1 / 2} \leq \operatorname{Im} \widehat{m}_{f c}(z) \leq C N^{-1 / 2} .
$$

Proof. Recall that

$$
\widehat{R}_{2}(z)=\frac{\operatorname{Im} \widehat{m}_{f c}(z)}{\eta_{0}+\operatorname{Im} \widehat{m}_{f c}(z)}=\frac{1}{N} \sum_{i=1}^{N} \frac{1}{\left|\lambda v_{i}-z-\widehat{m}_{f c}(z)\right|^{2}}<1, \quad\left(z \in \mathbb{C}^{+}\right),
$$

c.f., (4.15). For given $z \in \mathcal{D}_{\epsilon}^{\prime}$ with $\operatorname{Im} z=\eta_{0}$, choose $k \in \llbracket 1, n_{0}-1 \rrbracket$ such that (4.8) is satisfied. In the first case, where $\left|z-\widehat{z}_{k}\right| \gg N^{-1 / 2+2 \epsilon}$, we find from Lemma 4.2 and Lemma 4.5 that

$$
\left|\lambda v_{k}-\operatorname{Re}\left(z+\widehat{m}_{f c}(z)\right)\right| \gg N^{-1 / 2+2 \epsilon} .
$$


Since $z=E+\mathrm{i} \eta_{0}$ satisfies (4.8), we also find that

$$
\widehat{R}_{2}^{(k)}(z):=\frac{1}{N} \sum_{i}^{(k)} \frac{1}{\left|\lambda v_{i}-z-\widehat{m}_{f c}(z)\right|^{2}}=\frac{1}{N} \sum_{i}^{(k)} \frac{1}{\left|\lambda v_{i}-z-m_{f c}(z)\right|^{2}}+o(1)<c<1,
$$

for some constant $c$. Thus,

$$
\widehat{R}_{2}(z)=\frac{1}{N} \frac{1}{\left|\lambda v_{k}-z-\widehat{m}_{f c}(z)\right|^{2}}+\frac{1}{N} \sum_{i}^{(k)} \frac{1}{\left|\lambda v_{i}-z-\widehat{m}_{f c}(z)\right|^{2}}<c^{\prime}<1,
$$

for some constant $c^{\prime}$. Recalling that

$$
\operatorname{Im} \widehat{m}_{f c}(z)=\frac{\widehat{R}_{2}(z)}{1-\widehat{R}_{2}(z)} \eta_{0}
$$

statement (1) of the lemma follows.

Next, we consider the second case: $z=\widehat{z}_{k}=\widehat{E}_{k}+\mathrm{i} \eta_{0}$, for some $k \in \llbracket 1, n_{0}-1 \rrbracket$. We have

$$
\operatorname{Im} \widehat{m}_{f c}\left(\widehat{z}_{k}\right)=\frac{1}{N} \sum_{i=1}^{N} \frac{\eta_{0}+\operatorname{Im} \widehat{m}_{f c}\left(\widehat{z}_{k}\right)}{\left|\lambda v_{i}-\widehat{z}_{k}-\widehat{m}_{f c}\left(\widehat{z}_{k}\right)\right|^{2}}=\frac{1}{N} \frac{1}{\eta_{0}+\operatorname{Im} \widehat{m}_{f c}\left(\widehat{z}_{k}\right)}+\frac{1}{N} \sum_{i}^{(k)} \frac{\eta_{0}+\operatorname{Im} \widehat{m}_{f c}\left(\widehat{z}_{k}\right)}{\left|\lambda v_{i}-\widehat{z}_{k}-\widehat{m}_{f c}\left(\widehat{z}_{k}\right)\right|^{2}},
$$

hence

$$
\left(1-\widehat{R}_{2}^{(k)}\left(\widehat{z}_{k}\right)\right)\left(\operatorname{Im} \widehat{m}_{f c}\left(\widehat{z}_{k}\right)\right)^{2}+\left(1-2 \widehat{R}_{2}^{(k)}\left(\widehat{z}_{k}\right)\right) \eta_{0} \operatorname{Im} \widehat{m}_{f c}\left(\widehat{z}_{k}\right)=\frac{1}{N}+\widehat{R}_{2}^{(k)}\left(\widehat{z}_{k}\right) \eta_{0}^{2}
$$

Solving the quadratic equation above for $\operatorname{Im} \widehat{m}_{f c}\left(\widehat{z}_{k}\right)$, we find

$$
C^{-1} N^{-1 / 2} \leq \operatorname{Im} \widehat{m}_{f c}\left(\widehat{z}_{k}\right) \leq C N^{-1 / 2},
$$

completing the proof of the lemma.

Remark 5.3. For any $z=E+\mathrm{i} \eta_{0} \in \mathcal{D}_{\epsilon}^{\prime}$, we have, similarly to (5.8), that

$$
\operatorname{Im} \widehat{m}_{f c}(z) \leq \frac{1}{N} \frac{1}{\eta_{0}+\operatorname{Im} \widehat{m}_{f c}(z)}+\frac{1}{N} \sum_{i}^{(k)} \frac{\eta_{0}+\operatorname{Im} \widehat{m}_{f c}(z)}{\left|\lambda v_{i}-z-\widehat{m}_{f c}(z)\right|^{2}} .
$$

Solving this inequality for $\operatorname{Im} \widehat{m}_{f c}(z)$, we find that $\operatorname{Im} \widehat{m}_{f c}(z) \leq C N^{-1 / 2}$.

Recall that by Schur's complement formula we have, for all $i \in \llbracket 1, N \rrbracket$,

$$
G_{i i}=\frac{1}{\lambda v_{i}+w_{i i}-z-\sum_{s, t}^{(i)} h_{i s} G_{s t}^{(i)} h_{t i}} ;
$$

see (3.7). Define $\mathbb{E}_{i}$ to be the partial expectation with respect to the $i$-th column/row of $W$ and set

$$
Z_{i}:=\left(\mathbb{1}-\mathbb{E}_{i}\right) \sum_{s, t}^{(i)} h_{i s} G_{s t}^{(i)} h_{t i}=\sum_{s}^{(i)}\left(\left|w_{i s}\right|^{2}-\frac{1}{N}\right) G_{s s}^{(i)}+\sum_{s \neq t}^{(i)} w_{i s} G_{s t}^{(i)} w_{t i} .
$$

Using $Z_{i}$, we can rewrite $G_{i i}$ as

$$
G_{i i}=\frac{1}{\lambda v_{i}+w_{i i}-z-m^{(i)}-Z_{i}} .
$$

The following lemma states an a priori bound on $\operatorname{Im} m$, the imaginary part of $m=N^{-1} \operatorname{Tr} G$.

Lemma 5.4. We have with $(\xi, \nu)$-high probability on $\Omega_{V}$ that, for all $z=E+\mathrm{i} \eta_{0} \in \mathcal{D}_{\epsilon}^{\prime}$,

$$
\operatorname{Im} m(z) \leq \frac{N^{2 \epsilon}}{\sqrt{N}} .
$$


Proof. Fix $\eta=\eta_{0}$. For given $z=E+\mathrm{i} \eta_{0} \in \mathcal{D}_{\epsilon}^{\prime}$, choose $k \in \llbracket 1, n_{0}-1 \rrbracket$ such that (4.8) is satisfied. Suppose that $\operatorname{Im} m(z)>N^{-1 / 2+5 \epsilon / 3}$. Reasoning as in the proof of Lemma 4.5, we find the following equation for $\left(m-\widehat{m}_{f c}\right)$ :

$$
\begin{aligned}
m-\widehat{m}_{f c} & =\frac{1}{N} \sum_{i=1}^{N}\left(\frac{1}{\lambda v_{i}+w_{i i}-z-m^{(i)}-Z_{i}}-\frac{1}{\lambda v_{i}-z-\widehat{m}_{f c}}\right) \\
& =\frac{1}{N} \sum_{i=1}^{N} \frac{m^{(i)}-\widehat{m}_{f c}+Z_{i}-w_{i i}}{\left(\lambda v_{i}+w_{i i}-z-m^{(i)}-Z_{i}\right)\left(\lambda v_{i}-z-\widehat{m}_{f c}\right)} .
\end{aligned}
$$

Abbreviate

$$
T_{m} \equiv T_{m}(z):=\frac{1}{N} \sum_{i=1}^{N}\left|\frac{1}{\left(\lambda v_{i}+w_{i i}-z-m^{(i)}-Z_{i}\right)\left(\lambda v_{i}-z-\widehat{m}_{f c}\right)}\right| .
$$

We are going to show that $T_{m}<c<1$ : We define events $\Omega_{Z}$ and $\Omega_{W}$ by

$$
\Omega_{Z}(z) \equiv \Omega_{Z}:=\bigcap_{i=1}^{N}\left\{\left|Z_{i}\right| \leq\left(\varphi_{N}\right)^{\xi} \sqrt{\frac{\operatorname{Im} m^{(i)}}{N \eta}}\right\}, \quad \Omega^{W}:=\bigcap_{i=1}^{N}\left\{\left|w_{i i}\right| \leq \frac{\left(\varphi_{N}\right)^{\xi}}{\sqrt{N}}\right\} .
$$

We notice that, by the large deviation estimates in Lemma 3.4 and the subexponential decay of $\left|w_{i i}\right|, \Omega_{Z}$ and $\Omega_{W}$ both hold with high probability. Suppose now that $\Omega_{Z}$ and $\Omega_{W}$ hold. Then, we have

$$
\left|Z_{i}\right| \leq\left(\varphi_{N}\right)^{\xi} \sqrt{\frac{\operatorname{Im} m^{(i)}}{N \eta}} \leq\left(\varphi_{N}\right)^{-\xi} \operatorname{Im} m^{(i)}+C \frac{\left(\varphi_{N}\right)^{2 \xi}}{N \eta} \ll \operatorname{Im} m
$$

where we have used $\left|m-m^{(i)}\right| \leq C(N \eta)^{-1} \ll \operatorname{Im} m$. We also have from $\eta \ll \operatorname{Im} m$ that

$$
\operatorname{Im} m^{(i)}+\eta+\operatorname{Im} Z_{i}=(1+o(1)) \operatorname{Im} m .
$$

Thus,

$$
\begin{aligned}
\frac{1}{N} \sum_{i=1}^{N} \frac{1}{\left|\lambda v_{i}+w_{i i}-z-m^{(i)}-Z_{i}\right|^{2}} & =\frac{1}{N} \sum_{i=1}^{N} \frac{\operatorname{Im} G_{i i}}{\operatorname{Im} m^{(i)}+\eta+\operatorname{Im} Z_{i}}=\frac{1}{N} \sum_{i=1}^{N} \frac{\operatorname{Im} G_{i i}}{\operatorname{Im} m}(1+o(1)) \\
& =1+o(1) .
\end{aligned}
$$

We get from Lemma 4.5 that, on $\Omega_{V}$,

$$
\frac{1}{N} \sum_{i}^{(k)} \frac{1}{\left|\lambda v_{i}-z-\widehat{m}_{f c}\right|^{2}}=\frac{1}{N} \sum_{i}^{(k)} \frac{1+o(1)}{\left|\lambda v_{i}-z-m_{f c}\right|^{2}}<c<1,
$$

for some constant $c>0$, and

$$
\frac{1}{N}\left|\frac{1}{\left(\lambda v_{k}+w_{k k}-z-m^{(k)}-Z_{k}\right)\left(\lambda v_{k}-z-\widehat{m}_{f c}\right)}\right| \leq C \frac{1}{N} \frac{1}{N^{-1 / 2+5 \epsilon / 3} \eta} \leq N^{-2 \epsilon / 3} .
$$

Hence, we find that $T_{m}<c^{\prime}<1$ for some constant $c^{\prime}$. Notice that the assumption $\operatorname{Im} m>N^{-1 / 2+5 \epsilon / 3}$ also implies that

as we can see from Remark 5.3. Now, if we let

$$
\left|m-\widehat{m}_{f c}\right| \geq\left|\operatorname{Im} m-\operatorname{Im} \widehat{m}_{f c}\right|>C N^{-1 / 2+5 \epsilon / 3},
$$

$$
M:=\max _{i}\left|m^{(i)}-m+Z_{i}-w_{i i}\right|
$$

then $M \ll\left|m-\widehat{m}_{f c}\right|$. Thus, taking absolute value on both sides of (5.13), we get

$$
\left|m-\widehat{m}_{f c}\right| \leq T_{m}\left(\left|m-\widehat{m}_{f c}\right|+M\right)=\left(T_{m}+o(1)\right)\left|m-\widehat{m}_{f c}\right|,
$$

contradicting $T_{m}<c^{\prime}<1$.

We have thus shown that for fixed $z \in \mathcal{D}_{\epsilon}^{\prime}$,

$$
\operatorname{Im} m(z) \leq N^{-1 / 2+5 \epsilon / 3},
$$

with high probability on $\Omega_{V}$.

In order to prove that the desired bound holds uniformly on $z$, we consider a lattice $\mathcal{L}$ such that, for any $z$ satisfying the assumption of the lemma, there exists $z^{\prime}=E^{\prime}+\mathrm{i} \eta_{0} \in \mathcal{L}$ with $\left|z-z^{\prime}\right| \leq N^{-3}$. We have already seen that the uniform bound holds for all points in $\mathcal{L}$. For a point $z \notin \mathcal{L}$, we have $\left|m(z)-m\left(z^{\prime}\right)\right| \leq \eta_{0}^{2}\left|z-z^{\prime}\right| \leq N^{-1}$, for $z^{\prime} \in \mathcal{L}$ with $\left|z-z^{\prime}\right| \leq N^{-3}$. This proves the desired lemma. 
As a corollary of Lemma 5.4 we obtain:

Corollary 5.5. We have with $(\xi, \nu)$-high probability on $\Omega_{V}$ that, for all $z=E+\mathrm{i} \eta_{0} \in \mathcal{D}_{\epsilon}^{\prime}$,

$$
\max _{i}\left|Z_{i}(z)\right| \leq \frac{N^{2 \epsilon}}{\sqrt{N}}, \quad \max _{i}\left|Z_{i}^{(k)}(z)\right| \leq \frac{N^{2 \epsilon}}{\sqrt{N}}, \quad(k \in \llbracket 1, N \rrbracket) .
$$

Next, we prove an estimate for the difference $\Lambda(z):=\left|m(z)-\widehat{m}_{f c}(z)\right|$. We first show the bound on $\Lambda(z)$ in Proposition 5.1 holds for large $\eta$; see Lemma 5.6 below. Then, using the self-consistent equation (5.13), we show that if, for some $z \in \mathcal{D}_{\epsilon}^{\prime}, \Lambda(z) \leq N^{-1 / 2+3 \epsilon}$, then we must have $\Lambda(z) \leq N^{-1 / 2+2 \epsilon}$ with high probability; see Lemma 5.7. Thus, using the Lipschitz continuity of the Green function $G$ and of the Stieltjes transform $\widehat{m}_{f c}$, we can conclude that if $\Lambda(z) \leq N^{-1 / 2+2 \epsilon}$, we also have $\Lambda\left(z^{\prime}\right) \leq N^{-1 / 2+2 \epsilon}$, with high probability, for $z^{\prime}$ in a sufficiently small neighborhood of $z$. Repeated use this argument yields a proof of Proposition 5.1 at the end of this subsection.

Recall that we have set $\kappa_{0}=N^{-1 /(\mathrm{b}+1)}$; see (4.5).

Lemma 5.6. We have with high probability on $\Omega_{V}$ that, for all $z=E+\mathrm{i} \eta \in \mathcal{D}_{\epsilon}^{\prime}$ with $N^{-1 / 2+\epsilon} \leq \eta \leq N^{\epsilon} \kappa_{0}$,

$$
\left|m(z)-\widehat{m}_{f c}(z)\right| \leq \frac{N^{2 \epsilon}}{\sqrt{N}} .
$$

Proof. The proof closely follows the proof of Lemma 5.4. Fix $z \in \mathcal{D}_{\epsilon}^{\prime}$. Suppose that $\left|m(z)-\widehat{m}_{f c}(z)\right|>N^{-1 / 2+5 \epsilon / 3}$. Consider the self-consistent equation (5.13) and define $T_{m}$ as in (5.14).

Since $\operatorname{Im} m(E+\mathrm{i} \eta) \geq C \eta$, for $z \in \mathcal{D}_{\epsilon}^{\prime}$, with high probability on $\Omega_{V}$, we obtain that

$$
\operatorname{Im} m^{(i)}+\eta+\operatorname{Im} Z_{i}=(1+o(1)) \operatorname{Im} m,
$$

with high probability on $\Omega_{V}$, as in the proof of Lemma 5.4. This implies that $T_{m}<c<1$. If we let

$$
M=\max _{i}\left|m^{(i)}-m+Z_{i}-w_{i i}\right|,
$$

it then follows that $M \ll\left|m-\widehat{m}_{f c}\right|$ with high probability on $\Omega_{V}$. Taking absolute values on both sides of (5.13), we obtain a contradiction to the assumption $\left|m(z)-\widehat{m}_{f c}(z)\right|>N^{-1 / 2+5 \epsilon / 3}$. In order to attain a uniform bound, we again use the lattice argument as in the proof of Lemma 5.4. This completes the proof of the lemma.

Lemma 5.7. Let $z \in \mathcal{D}_{\epsilon}^{\prime}$. If $\left|m(z)-\widehat{m}_{f c}(z)\right| \leq N^{-1 / 2+3 \epsilon}$, then we have with $(\xi, \nu)$-high probability on $\Omega_{V}$ that $\left|m(z)-\widehat{m}_{f c}(z)\right| \leq N^{-1 / 2+2 \epsilon}$.

Proof. Since the proof closely follows the proof of Lemma 5.4, we only check the main steps here. Fix $z \in \mathcal{D}_{\epsilon}^{\prime}$ and choose $k \in \llbracket 1, n_{0}-1 \rrbracket$ such that (4.8) is satisfied. Assume that $N^{-1 / 2+5 \epsilon / 3}<\left|m(z)-\widehat{m}_{f c}(z)\right| \leq N^{-1 / 2+3 \epsilon}$. We consider the self-consistent equation (5.13) and define $T_{m}$ as in (5.14). We now estimate $T_{m}$. For $i \neq k, i \in \llbracket 1, N \rrbracket$, we have

$$
\frac{1}{\left(\lambda v_{i}+w_{i i}-z-m^{(i)}-Z_{i}\right)\left(\lambda v_{i}-z-\widehat{m}_{f c}\right)}=\frac{1}{\left(\lambda v_{i}-z-\widehat{m}_{f c}\right)^{2}}+o(1),
$$

where we have used that

$$
\begin{aligned}
\left|w_{i i}-m^{(i)}-Z_{i}+\widehat{m}_{f c}\right| & \leq\left|w_{i i}\right|+\left|m-m^{(i)}\right|+\left|m-\widehat{m}_{f c}\right|+\left|Z_{i}\right| \\
& \leq \frac{N^{\epsilon}}{\sqrt{N}}+\frac{C}{N \eta}+N^{-1 / 2+3 \epsilon}+C\left(\varphi_{N}\right)^{\xi} \sqrt{\frac{\operatorname{Im} m^{(i)}}{N \eta}} \\
& \ll\left|v_{i}-v_{k}\right|,
\end{aligned}
$$

which holds with high probability on $\Omega_{V}$. For $i=k$, we have

$$
\begin{aligned}
\mid \lambda v_{k}+w_{k k} & -z-m^{(k)}-Z_{k}|+| \lambda v_{k}-z-\widehat{m}_{f c} \mid \\
& \geq\left|m-\widehat{m}_{f c}\right|-\left|w_{k k}\right|-\left|m-m^{(k)}\right|-\left|Z_{k}\right| \\
& \geq \frac{1}{2} N^{-1 / 2+2 \epsilon}
\end{aligned}
$$

thus, as in the proofs of Lemma 4.5 and Lemma 5.4 ,

$$
\frac{1}{N}\left|\frac{1}{\left(\lambda v_{k}+w_{k k}-z-m^{(k)}-Z_{k}\right)\left(\lambda v_{k}-z-\widehat{m}_{f c}\right)}\right| \leq C N^{-2 \epsilon / 3},
$$

where we used that $\left|G_{k k}\right|,\left|g_{k}\right| \leq \eta^{-1}$. 
We now have that

$$
T_{m}=\widehat{R}_{2}^{(k)}+o(1)=R_{2}+o(1)
$$

and, in particular, $T_{m}<c<1$, with high probability on $\Omega_{V}$. We again let $M:=\max _{i}\left|m^{(i)}-m+Z_{i}-w_{i i}\right|$ and find that $M \ll\left|m-\widehat{m}_{f c}\right|$ with high probability on $\Omega_{V}$, which contradicts the assumption. Therefore, if $\left|m(z)-\widehat{m}_{f c}(z)\right| \leq N^{-1 / 2+3 \epsilon}$, then $\left|m(z)-\widehat{m}_{f c}(z)\right| \leq N^{-1 / 2+5 \epsilon / 3}$ with high probability on $\Omega_{V}$. In order to attain a uniform bound, we use the lattice argument as in the proof of Lemma 5.4. This proves the desired lemma.

We now prove Proposition 5.1 using a discrete continuity argument.

Proof of Proposition 5.1. Fix $E$ such that $z=E+\mathrm{i} \eta_{0} \in \mathcal{D}_{\epsilon}^{\prime}$. Consider a sequence $\left(\eta_{j}\right)$ defined by $\eta_{j=0}=\eta_{0}$ and $\eta_{j}=\eta_{j-1}+N^{-2}$. Let $K$ be the smallest positive integer such that $\eta_{K} \geq N^{-1 / 2+\epsilon}$. We prove by induction that, for $z_{j}=E+\mathrm{i} \eta_{j}$, we have with high probability on $\Omega_{V}$ that

$$
\left|m\left(z_{j}\right)-\widehat{m}_{f c}\left(z_{j}\right)\right| \leq \frac{N^{2 \epsilon}}{\sqrt{N}} .
$$

The case $j=K$ is already proved in Lemma [5.6. For any $z=E+\mathrm{i} \eta$, with $\eta_{j-1} \leq \eta \leq \eta_{j}$, we have

$$
\left|m\left(z_{j}\right)-m(z)\right| \leq \frac{\left|z_{j}-z\right|}{\eta_{j-1}^{2}} \leq \frac{N^{2 \epsilon}}{N}, \quad\left|\widehat{m}_{f c}\left(z_{j}\right)-\widehat{m}_{f c}(z)\right| \leq \frac{\left|z_{j}-z\right|}{\eta_{j-1}^{2}} \leq \frac{N^{2 \epsilon}}{N}
$$

Thus, we find that if $\left|\widehat{m}_{f c}\left(z_{j}\right)-m\left(z_{j}\right)\right| \leq N^{-1 / 2+2 \epsilon}$ then

$$
\left|m(z)-\widehat{m}_{f c}(z)\right| \leq N^{-1 / 2+2 \epsilon}+\frac{2 N^{2 \epsilon}}{N} \ll N^{-1 / 2+3 \epsilon} .
$$

We now invoke Lemma 5.7 to obtain that $\left|m(z)-\widehat{m}_{f c}(z)\right| \leq N^{-1 / 2+2 \epsilon}$. This proves the desired lemma for any $z=E+\mathrm{i} \eta$, with $\eta_{j-1} \leq \eta \leq \eta_{j}$. The desired lemma can now be proved by induction on $j$. Uniformity can now be obtained using a lattice argument.

5.2. Estimates on $\left|m-m^{(i)}\right|$. In order to derive a more accurate estimate on the difference $\left|\operatorname{Im} m(z)-\operatorname{Im} \widehat{m}_{f c}(z)\right|$, as the one obtained in Proposition [5.1 we establish detailed estimates on $\left|m-m^{(i)}\right|$ and $N^{-1} \sum Z_{i}$. We first prove the following bound on the difference $\left|m-m^{(i)}\right|$.

Lemma 5.8. There exists a constant $C>1$ such that the following bound holds with $(\xi, \nu)$-high probability on $\Omega_{V}$ for all $z=E+\mathrm{i} \eta_{0} \in \mathcal{D}_{\epsilon}^{\prime}$ : For given $z$, choose $k \in \llbracket 1, n_{0}-1 \rrbracket$ such that (4.8) is satisfied. Then, for any $i \neq k$, $i \in \llbracket 1, N \rrbracket$,

$$
\left|m(z)-m^{(i)}(z)\right| \leq C N^{1 /(\mathrm{b}+1)} \frac{N^{4 \epsilon}}{N}
$$

and

$$
\left|m^{(k)}(z)-m^{(k i)}(z)\right| \leq C N^{1 /(\mathrm{b}+1)} \frac{N^{4 \epsilon}}{N}
$$

Proof. Let $\eta=\eta_{0}$. Since

$$
G_{i j}=-G_{i i}\left(\sum_{s}^{(i)} w_{i s} G_{s j}^{(i)}\right)
$$

we find from the large deviation estimates in Lemma 3.4 and the Ward identity 3.11) that

$$
\left|G_{j j}-G_{j j}^{(i)}\right|=\left|\frac{G_{i j} G_{j i}}{G_{i i}}\right| \leq\left(\varphi_{N}\right)^{2 \xi}\left|G_{i i}\right| \frac{\operatorname{Im} G_{j j}^{(i)}}{N \eta}
$$

with high probability on $\Omega_{V}$. For $i \neq k$, we have

$$
\left|G_{i i}\right|=\frac{1}{\left|\lambda v_{i}+w_{i i}-z-m^{(i)}-Z_{i}\right|} \leq \frac{C}{\left|\lambda v_{i}-z-\widehat{m}_{f c}\right|} \leq C N^{\epsilon} \kappa_{0}^{-1},
$$


with high probability on $\Omega_{V}$. Thus, we obtain

$$
\begin{aligned}
\left|m(z)-m^{(i)}(z)\right| & \leq \frac{\left|G_{i i}\right|}{N}+\frac{1}{N} \sum_{j}^{(i)}\left|G_{j j}-G_{j j}^{(i)}\right| \leq \frac{\left|G_{i i}\right|}{N}+C\left(\varphi_{N}\right)^{2 \xi} \frac{N^{\epsilon} \kappa_{0}^{-1}}{N} \sum_{j}^{(i)} \frac{\operatorname{Im} G_{j j}^{(i)}}{N \eta} \\
& \leq \frac{N^{\epsilon} \kappa_{0}^{-1}}{N}+C\left(\varphi_{N}\right)^{2 \xi} \frac{N^{\epsilon} \kappa_{0}^{-1}}{N \eta} \operatorname{Im} m^{(i)} \leq C N^{1 /(\mathrm{b}+1)} \frac{N^{4 \epsilon}}{N}
\end{aligned}
$$

with high probability on $\Omega_{V}$. Together with the usual lattice argument, this proves the first part of the lemma. The second part of the lemma can be proved in a similar manner.

5.3. Estimates on $N^{-1} \sum Z_{i}$. Recall that $n_{0}>10$ is an integer independent of $N$. In the next lemma, we control the fluctuation average $\frac{1}{N} \sum_{i=n_{0}}^{N} Z_{i}$ and other related quantities. Here, we aim to use cancellations in the averaging over $i$, but note that $\left(Z_{i}\right)$ are not independent.

Lemma 5.9. There is a constant $c$, such that, for all $z \in \mathcal{D}_{\epsilon}^{\prime}$, the following bounds hold with $(\xi-2, \nu)$-high probability on $\Omega_{V}$ :

$$
\left|\frac{1}{N} \sum_{i=n_{0}}^{N} Z_{i}(z)\right| \leq\left(\varphi_{N}\right)^{c \xi} N^{-1 / 2-\mathfrak{b} / 2+4 \epsilon},
$$

and, for $k \in \llbracket 1, n_{0}-1 \rrbracket$,

$$
\left|\frac{1}{N} \sum_{\substack{i=n_{0} \\ i \neq k}}^{N} Z_{i}^{(k)}(z)\right| \leq\left(\varphi_{N}\right)^{c \xi} N^{-1 / 2-\mathfrak{b} / 2+4 \epsilon} .
$$

Corollary 5.10. There is a constant $c$, such that, for all $z \in \mathcal{D}_{\epsilon}^{\prime}$, the following bounds hold with $(\xi-2, \nu)$-high probability on $\Omega_{V}$ :

$$
\left|\frac{1}{N} \sum_{i=n_{0}}^{N} \frac{w_{i i}-Z_{i}(z)}{\left(\lambda v_{i}-z-\widehat{m}_{f c}(z)\right)^{2}}\right| \leq\left(\varphi_{N}\right)^{c \xi} N^{-1 / 2-\mathfrak{b} / 2+4 \epsilon},
$$

and, for $k \in \llbracket 1, n_{0}-1 \rrbracket$,

$$
\left|\frac{1}{N} \sum_{\substack{i=n_{0} \\ i \neq k}}^{N} \frac{w_{i i}-Z_{i}^{(k)}(z)}{\left(\lambda v_{i}-z-\widehat{m}_{f c}(z)\right)^{2}}\right| \leq\left(\varphi_{N}\right)^{c \xi} N^{-1 / 2-\mathfrak{b} / 2+4 \epsilon} .
$$

Remark 5.11. The bounds we obtained in Lemma [5.8, Lemma [5.9] and Corollary [5.10 are $o(\eta)$. This will be used on several occasions in the next subsection.

Lemma 5.9 and Corollary 5.10 are proved in Section 6 .

5.4. Proof of Proposition 5.1. Recall the definition of $\left(\widehat{z}_{k}\right)$ in (5.1). We first estimate $\operatorname{Im} m(z)$ for $z=E+\mathrm{i} \eta_{0}$ satisfying $\left|z-\widehat{z}_{k}\right| \geq N^{-1 / 2+3 \epsilon}$, for all $k \in \llbracket 1, n_{0}-1 \rrbracket$.

Lemma 5.12. There exists a constant $C>1$ such that the following bound holds with $(\xi-2, \nu)$-high probability on $\Omega_{V}$ : For any $z=E+\mathrm{i} \eta_{0} \in \mathcal{D}_{\epsilon}^{\prime}$, satisfying $\left|z-\widehat{z}_{k}\right| \geq N^{-1 / 2+3 \epsilon}$ for all $k \in \llbracket 1, n_{0}-1 \rrbracket$, we have

$$
C^{-1} \eta \leq \operatorname{Im} m(z) \leq C \eta .
$$

Proof. Let $z \in \mathcal{D}_{\epsilon}^{\prime}$ with $\eta=\eta_{0}$ and choose $k \in \llbracket 1, n_{0}-1 \rrbracket$ such that (4.8) is satisfied. Consider

$$
m=\frac{G_{k k}}{N}+\frac{1}{N} \sum_{i}^{(k)} \frac{1}{\lambda v_{i}+w_{i i}-z-m^{(i)}-Z_{i}} .
$$

From the assumption in (4.8), Corollary [5.5, and Proposition 5.1 we find that, with high probability on $\Omega_{V}$,

$$
\begin{aligned}
& \left|\frac{1}{N} \sum_{i}^{(k)}\left(\frac{1}{\lambda v_{i}+w_{i i}-z-m^{(i)}-Z_{i}}-\frac{1}{\lambda v_{i}-z-\widehat{m}_{f c}}-\frac{m^{(i)}-\widehat{m}_{f c}+Z_{i}-w_{i i}}{\left(\lambda v_{i}-z-\widehat{m}_{f c}\right)^{2}}\right)\right| \\
& \leq \frac{C}{N} \sum_{i}^{(k)} \frac{N^{-1+4 \epsilon}}{\left|\lambda v_{i}-z-\widehat{m}_{f c}\right|^{3}} \leq C \frac{N^{4 \epsilon}}{N} N^{\epsilon} N^{1 /(\mathrm{b}+1)} \frac{1}{N} \sum_{i}^{(k)} \frac{1}{\left|\lambda v_{i}-z-\widehat{m}_{f c}\right|^{2}} \ll \eta .
\end{aligned}
$$


We also observe that

$$
\left|\frac{1}{N} \sum_{\substack{i=1 \\ i \neq k}}^{n_{0}} \frac{w_{i i}-Z_{i}}{\left(\lambda v_{i}-z-\widehat{m}_{f c}(z)\right)^{2}}\right| \leq C N^{-1} N^{-1 / 2+2 \epsilon} N^{1 /(\mathrm{b}+1)} \ll N^{-1} \ll \eta .
$$

Thus, from Lemma 5.8 and Corollary 5.10, we find with high probability on $\Omega_{V}$ that

$$
\frac{1}{N} \sum_{i}^{(k)} \frac{m^{(i)}-\widehat{m}_{f c}+Z_{i}-w_{i i}}{\left(\lambda v_{i}-z-\widehat{m}_{f c}\right)^{2}}=\frac{1}{N} \sum_{i}^{(k)} \frac{m-\widehat{m}_{f c}}{\left(\lambda v_{i}-z-\widehat{m}_{f c}\right)^{2}}+o(\eta)
$$

Recalling (5.5), i.e.,

$$
\left|\lambda v_{k}-\operatorname{Re}\left(z+\widehat{m}_{f c}(z)\right)\right| \gg N^{-1 / 2+2 \epsilon},
$$

we get $\left|G_{k k}\right| \leq N^{1 / 2-2 \epsilon}$. We thus obtain from (5.31), (5.32), and (5.33) that, with high probability on $\Omega_{V}$,

$$
m=\frac{1}{N} \sum_{i}^{(k)}\left(\frac{1}{\lambda v_{i}-z-\widehat{m}_{f c}}+\frac{m-\widehat{m}_{f c}}{\left(\lambda v_{i}-z-\widehat{m}_{f c}\right)^{2}}\right)+o(\eta)
$$

We also notice that, with high probability on $\Omega_{V}$,

$$
\frac{1}{N} \sum_{i}^{(k)} \frac{1}{\lambda v_{i}-z-m}=\frac{1}{N} \sum_{i}^{(k)}\left(\frac{1}{\lambda v_{i}-z-\widehat{m}_{f c}}+\frac{m-\widehat{m}_{f c}}{\left(\lambda v_{i}-z-\widehat{m}_{f c}\right)^{2}}\right)+\mathcal{O}\left(\frac{1}{N} \sum_{i}^{(k)} \frac{N^{-1+4 \epsilon}}{\left|\lambda v_{i}-z-\widehat{m}_{f c}\right|^{3}}\right)
$$

and following the estimate in (5.32), we find that

$$
\frac{1}{N} \sum_{i}^{(k)} \frac{1}{\lambda v_{i}-z-m}=\frac{1}{N} \sum_{i}^{(k)}\left(\frac{1}{\lambda v_{i}-z-\widehat{m}_{f c}}+\frac{m-\widehat{m}_{f c}}{\left(\lambda v_{i}-z-\widehat{m}_{f c}\right)^{2}}\right)+o(\eta)=m+o(\eta) .
$$

Taking imaginary parts, we get

$$
\operatorname{Im} m=\frac{1}{N} \sum_{i}^{(k)} \frac{\eta+\operatorname{Im} m}{\left|\lambda v_{i}-z-m\right|^{2}}+o(\eta)
$$

Since

$$
\frac{1}{N} \sum_{i}^{(k)} \frac{1}{\left|\lambda v_{i}-z-m\right|^{2}}=\frac{1}{N} \sum_{i}^{(k)} \frac{1}{\left|\lambda v_{i}-z-m_{f c}\right|^{2}}+o(1)<c<1
$$

for some constant $c$, we can conclude that $C^{-1} \eta \leq \operatorname{Im} m \leq C \eta$ with high probability for some $C>1$. This proves the desired lemma.

As a next step, we prove that there exists $\widetilde{z}_{k}=\widetilde{E}_{k}+\mathrm{i} \eta_{0}$ near $\widehat{z}_{k}$ such that $\operatorname{Im} m\left(\widetilde{z}_{k}\right) \gg \eta$. Before proving this, we first show that $\operatorname{Im} m^{(k)}(z) \sim \eta$ even if $z$ is near $\widehat{z}_{k}$.

Lemma 5.13. There exists a constant $C>1$ such that the following bound holds with $(\xi-2, \nu)$-high probability on $\Omega_{V}$, for all $z=E+\mathrm{i} \eta_{0} \in \mathcal{D}_{\epsilon}^{\prime}$ : For given $z$, choose $k \in \llbracket 1, n_{0}-1 \rrbracket$ such that (4.8) is satisfied. Then, we have

$$
C^{-1} \eta_{0} \leq \operatorname{Im} m^{(k)}(z) \leq C \eta_{0}
$$

Proof. Reasoning as in the proof of Lemma [5.12, we find from Proposition 5.1, Corollary [5.5, Lemma 5.8, and Corollary 5.10 that, with high probability on $\Omega_{V}$,

$$
m^{(k)}=\frac{1}{N} \sum_{i}^{(k)}\left(\frac{1}{\lambda v_{i}-z-\widehat{m}_{f c}}+\frac{m^{(k)}-\widehat{m}_{f c}}{\left(\lambda v_{i}-z-\widehat{m}_{f c}\right)^{2}}\right)+o\left(\eta_{0}\right)=\frac{1}{N} \sum_{i}^{(k)} \frac{1}{\lambda v_{i}-z-m^{(k)}}+o\left(\eta_{0}\right) .
$$

Considering the imaginary part, we can prove the desired lemma as in the proof of Lemma 5.12 .

Corollary 5.14. There exists a constant $C>1$ such that the following bound holds with $(\xi-2, \nu)$-high probability on $\Omega_{V}$, for all $z=E+\mathrm{i} \eta_{0} \in \mathcal{D}_{\epsilon}^{\prime}:$ For given $z$, choose $k \in \llbracket 1, n_{0}-1 \rrbracket$ such that (4.8) is satisfied. Then, we have

$$
\left|Z_{k}\right| \leq C \frac{\left(\varphi_{N}\right)^{\xi}}{\sqrt{N}}
$$


We are now ready to locate the points $z \in \mathcal{D}_{\epsilon}^{\prime}$ for which $\operatorname{Im} m(z) \gg \eta_{0}$.

Lemma 5.15. For any $k \in \llbracket 1, n_{0}-1 \rrbracket$, there exists $\widetilde{E}_{k} \in \mathbb{R}$ such that the following holds with $(\xi-2, \nu)$-high probability on $\Omega_{V}$ : If we let $\widetilde{z}_{k}:=\widetilde{E}_{k}+\mathrm{i} \eta_{0}$, then $\left|\widetilde{z}_{k}-\widehat{z}_{k}\right| \leq N^{-1 / 2+3 \epsilon}$ and $\operatorname{Im} m\left(\widetilde{z}_{k}\right) \gg \eta_{0}$.

Proof. We first notice that the condition $\left|z-\widehat{z}_{k}\right| \geq N^{-1 / 2+3 \epsilon}$ has not been used in the derivation of (5.32) and (5.33), hence, even though $\left|z-\widehat{z}_{k}\right| \leq N^{-1 / 2+3 \epsilon}$, we still attain that

$$
m=\frac{G_{k k}}{N}+\frac{1}{N} \sum_{i}^{(k)} \frac{1}{\lambda v_{i}+w_{i i}-z-m^{(i)}-Z_{i}}=\frac{G_{k k}}{N}+\frac{1}{N} \sum_{i}^{(k)} \frac{1}{\lambda v_{i}-z-m}+o\left(\eta_{0}\right),
$$

with high probability on $\Omega_{V}$. Consider

$$
\frac{1}{G_{k k}}=\lambda v_{k}+w_{k k}-z-m^{(k)}-Z_{k}
$$

Setting $z_{k}^{+}:=\widehat{z}_{k}+N^{-1 / 2+3 \epsilon}$, Lemma 4.2 shows that

$$
\operatorname{Re}\left(z_{k}^{+}+m_{f c}\left(z_{k}^{+}\right)\right)-\operatorname{Re}\left(\widehat{z}_{k}+m_{f c}\left(\widehat{z}_{k}\right)\right) \geq C N^{-1 / 2+3 \epsilon},
$$

on $\Omega_{V}$. Thus, from Lemma 4.5 and the definition of $\widehat{z}_{k}$, we find that

$$
\lambda v_{k}-\operatorname{Re}\left(z_{k}^{+}+\widehat{m}_{f c}\left(z_{k}^{+}\right)\right) \leq-C N^{-1 / 2+3 \epsilon},
$$

on $\Omega_{V}$. Similarly, if we let $z_{k}^{-}:=\widehat{z}_{k}-N^{-1 / 2+3 \epsilon}$, we have that

$$
\lambda v_{k}-\operatorname{Re}\left(z_{k}^{-}+\widehat{m}_{f c}\left(z_{k}^{-}\right)\right) \geq C N^{-1 / 2+3 \epsilon},
$$

on $\Omega_{V}$. Since

$$
\left|w_{k k}+\widehat{m}_{f c}-m^{(k)}-Z_{k}\right| \leq\left|w_{k k}\right|+\left|m-\widehat{m}_{f c}\right|+\left|m^{(k)}-m\right|+\left|Z_{k}\right| \ll N^{-1 / 2+3 \epsilon},
$$

with high probability on $\Omega_{V}$, we find that there exists $\widetilde{z}_{k}=\widetilde{E}_{k}+\mathrm{i} \eta_{0}$, with $\widetilde{E}_{k} \in\left(\widehat{E}_{k}-N^{-1 / 2+3 \epsilon}, \widehat{E}_{k}+N^{-1 / 2+3 \epsilon}\right)$, such that $\operatorname{Re} G_{k k}\left(\widetilde{z}_{k}\right)=0$. When $z=\widetilde{z}_{k}$, we have from Lemma 5.13 and Corollary 5.14 that, with high probability on $\Omega_{V}$,

$$
\left|\operatorname{Im} G_{k k}\left(\widetilde{z}_{k}\right)\right|=\frac{1}{\left|\operatorname{Im} \widetilde{z}_{k}+\operatorname{Im} m^{(k)}\left(\widetilde{z}_{k}\right)+\operatorname{Im} Z_{k}\left(\widetilde{z}_{k}\right)\right|} \geq C\left(\varphi_{N}\right)^{-\xi} N^{1 / 2}, \quad \operatorname{Re} G_{k k}\left(\widetilde{z}_{k}\right)=0
$$

From (5.40), we obtain that

$$
\operatorname{Im} m\left(\widetilde{z}_{k}\right)=\frac{\operatorname{Im} G_{k k}\left(\widetilde{z}_{k}\right)}{N}+\frac{1}{N} \sum_{i}^{(k)} \frac{\eta_{0}+\operatorname{Im} m\left(\widetilde{z}_{k}\right)}{\left|\lambda v_{i}-\widetilde{z}_{k}-m\left(\widetilde{z}_{k}\right)\right|^{2}}+o\left(\eta_{0}\right)
$$

Since

$$
\sum_{i}^{(k)} \frac{1}{\left|\lambda v_{i}-\widetilde{z}_{k}-m\left(\widetilde{z}_{k}\right)\right|^{2}}<c<1
$$

with high probability on $\Omega_{V}$, for some constant $c$, we get

$$
\operatorname{Im} m\left(\widetilde{z}_{k}\right) \geq C\left(\varphi_{N}\right)^{-\xi} N^{-1 / 2}+C \eta_{0} \gg \eta_{0},
$$

with high probability on $\Omega_{V}$, which was to be proved.

We now turn to the proof of Proposition 4.6. Recall that we denote by $\mu_{k}$ the $k$-th largest eigenvalue of $H$, $k \in \llbracket 1, n_{0}-1 \rrbracket$. Also recall that $\kappa_{0}=N^{-1 /(\mathrm{b}+1)} ;$ see (4.5).

Proof of Proposition 4.6. We first consider the case $k=1$. From the spectral decomposition of $H$, we have

$$
\operatorname{Im} m\left(E+\mathrm{i} \eta_{0}\right)=\frac{1}{N} \sum_{\alpha=1}^{N} \frac{\eta_{0}}{\left(\mu_{\alpha}-E\right)^{2}+\eta_{0}^{2}},
$$

and in particular, $\operatorname{Im} m\left(\mu_{1}+\mathrm{i} \eta_{0}\right) \geq\left(N \eta_{0}\right)^{-1} \gg \eta_{0}$. Recall that $\mu_{1} \leq 3+\lambda$ with high probability as discussed in (4.4). Recall the definition of $\widehat{z}_{1}=\widehat{E}_{1}+\mathrm{i} \eta_{0}$ in (5.1). Since, with high probability on $\Omega_{V}, \operatorname{Im} m(z) \sim \eta_{0}$ for $z \in \mathcal{D}_{\epsilon}^{\prime}$ satisfying $\left|z-\widehat{z}_{1}\right| \geq N^{-1 / 2+3 \epsilon}$, as we proved in Lemma 5.15, we obtain that $\mu_{1}<\widehat{E}_{1}+N^{-1 / 2+3 \epsilon}$. 
Recall the definitions for $\widehat{z}_{1}$ and $z_{1}^{-}$in the proof of Lemma 5.15. Assume that $\mu_{1}<\widehat{E}_{1}-N^{-1 / 2+3 \epsilon}$. Then, on the interval $\left(\widehat{E}_{1}-N^{-1 / 2+3 \epsilon}, \widehat{E}_{1}+N^{-1 / 2+3 \epsilon}\right), \operatorname{Im} m\left(E+\mathrm{i} \eta_{0}\right)$ is a decreasing function of $E$. However, we already showed in Lemma 5.12 and Lemma 5.15 that, with $(\xi-2, \nu)$-high probability, $\operatorname{Im} m\left(\widetilde{z}_{1}\right) \gg \eta_{0}, \operatorname{Im} m\left(z_{1}^{-}\right) \sim \eta_{0}$, and $\operatorname{Re} \widetilde{z}_{1}>\operatorname{Re} z_{1}^{-}$. Thus, $\mu_{1} \geq \widehat{E}_{1}-N^{-1 / 2+3 \epsilon}$. We now use Lemma 4.2 and Lemma 4.5, together with Remark[5.3] to conclude that

$$
\mu_{1}+\mathrm{i} \eta_{0}+\widehat{m}_{f c}\left(\mu_{1}+\mathrm{i} \eta_{0}\right)=\widehat{z}_{1}+\widehat{m}_{f c}\left(\widehat{z}_{1}\right)+\mathcal{O}\left(N^{-1 / 2+3 \epsilon}\right)=\lambda v_{1}+\mathcal{O}\left(N^{-1 / 2+3 \epsilon}\right),
$$

which proves the proposition for the special choice $k=1$.

Next, we consider the case $k=2$; the general case can be proved in a similar manner by induction. Consider $H^{(1)}$, the minor of $H$ obtained by removing the first column and the first row. If we denote by $\mu_{1}^{(1)}$ the largest eigenvalue of $H^{(1)}$, then the Cauchy interlacing property yields $\mu_{2} \leq \mu_{1}^{(1)}$. We now follow the first part of the proof to estimate $\mu_{1}^{(1)}$, which gives us

$$
\widehat{E}_{2}-N^{-1 / 2+3 \epsilon} \leq \mu_{1}^{(1)} \leq \widehat{E}_{2}+N^{-1 / 2+3 \epsilon},
$$

where we let $\widehat{z}_{2}=\widehat{E}_{2}+\mathrm{i} \eta_{0}$ be a solution to the equation

$$
\operatorname{Re}\left(\widehat{z}_{2}+\widehat{m}_{f c}\left(\widehat{z}_{2}\right)\right)=\lambda v_{2} .
$$

This, in particular, shows that

$$
\mu_{2} \leq \widehat{E}_{2}+N^{-1 / 2+3 \epsilon}
$$

To prove the lower bound, we may follow the arguments we used in the first part of the proof. Recall that we have proved in Lemma 5.12 and Lemma 5.15 that, with $(\xi-2, \nu)$-high probability on $\Omega_{V}$,

(1) for $z=\widehat{z}_{2}-N^{-1 / 2+3 \epsilon}$, we have $\operatorname{Im} m(z) \leq C \eta_{0}$;

(2) there exists $\widetilde{z}_{2}=\widetilde{E}_{2}+\mathrm{i} \eta_{0}$, satisfying $\left|\widetilde{z}_{2}-\widehat{z}_{2}\right| \leq N^{-1 / 2+3 \epsilon}$, such that $\operatorname{Im} m\left(\widetilde{z}_{2}\right) \gg \eta_{0}$.

If $\mu_{2}<\widehat{E}_{2}-N^{-1 / 2+3 \epsilon}$, then

$$
\operatorname{Im} m\left(E+\mathrm{i} \eta_{0}\right)-\frac{1}{N} \frac{\eta_{0}}{\left(\mu_{1}-E\right)^{2}+\eta_{0}^{2}}=\frac{1}{N} \sum_{\alpha=2}^{N} \frac{\eta_{0}}{\left(\mu_{\alpha}-E\right)^{2}+\eta_{0}^{2}}
$$

is a decreasing function of $E$. Since we know that, with $(\xi-2, \nu)$-high probability on $\Omega_{V}$,

$$
\frac{1}{N} \frac{\eta_{0}}{\left(\mu_{1}-\widehat{E}_{2}\right)^{2}+\eta_{0}^{2}} \leq \frac{1}{N} \frac{C \eta_{0}}{N^{-2 \epsilon} \kappa_{0}^{2}} \ll \eta_{0}
$$

we have that $\operatorname{Im} m\left(\widetilde{z}_{2}\right) \leq C \eta_{0}$, which contradicts the definition of $\widetilde{z}_{2}$. Thus, we find that $\mu_{2} \geq \widehat{E}_{2}-N^{-1 / 2+3 \epsilon}$, with $(\xi-2, \nu)$-high probability on $\Omega_{V}$.

We now proceed as above to conclude that, with $(\xi-2, \nu)$-high probability on $\Omega_{V}$,

$$
\mu_{2}+\mathrm{i} \eta_{0}+\widehat{m}_{f c}\left(\mu_{2}+\mathrm{i} \eta_{0}\right)=\widehat{z}_{2}+\widehat{m}_{f c}\left(\widehat{z}_{2}\right)+\mathcal{O}\left(N^{-1 / 2+3 \epsilon}\right)=\lambda v_{2}+\mathcal{O}\left(N^{-1 / 2+3 \epsilon}\right),
$$

which proves the proposition for $k=2$. The general case is proven in the same way.

\section{Fluctuation Average Lemma}

In this section we prove Lemma 5.9 and Corollary 5.10 . Recall that we denote by $\mathbb{E}_{i}$ the partial expectation with respect to the $i$-th column/row of $W$. Set $Q_{i}:=\mathbb{1}-\mathbb{E}_{i}$.

We are interested in bounding the fluctuation average

$$
\frac{1}{N} \sum_{a=n_{0}}^{N} Z_{a}(z),
$$


where $n_{0}$ is a $N$-independent fixed integer. We first note that, using Schur's complement formula, we can write

$$
\begin{aligned}
\frac{1}{N} \sum_{a=n_{0}}^{N} Q_{a}\left(\frac{1}{G_{a a}}\right) & =\frac{1}{N} \sum_{a=n_{0}}^{N} w_{a a}-\frac{1}{N} \sum_{a=n_{0}}^{N} Q_{a} \sum_{k, l}^{(a)} w_{a k} G_{k l}^{(a)} w_{l a} \\
& =-\frac{1}{N} \sum_{a=n_{0}}^{N} Z_{a}+\mathcal{O}\left(\frac{\left(\varphi_{N}\right)^{c \xi}}{N}\right)
\end{aligned}
$$

with high probability, where we used the large deviation estimate (3.14). The main result of this section, Lemma 6.6. asserts that

$$
\left|\frac{1}{N} \sum_{a=n_{0}}^{N} Q_{a}\left(\frac{1}{G_{a a}}\right)\right| \leq\left(\varphi_{N}\right)^{c \xi} N^{-1 / 2-\mathfrak{b} / 2+4 \epsilon},
$$

with $(\xi-2, \nu)$-high probability, provided that $z$ satisfies $\left|\lambda v_{a}-\operatorname{Re} m_{f c}(z)-\operatorname{Re} z\right| \geq \frac{1}{2} N^{-1 /(b+1)+\epsilon}$, for all $a \geq n_{0}$. (Note that we reduced here $\xi$ to $\xi-2$ ).

Fluctuation averages of the form (6.1) (with $n_{0}=1$ ) and more general fluctuation averages have been studied in [18, see also [17], for generalized Wigner ensembles and random band matrices. In [32, these ideas have been applied to the deformed Wigner ensemble, under the assumptions that the limiting eigenvalue distribution has a square root behavior at the spectral edge. In these studies, it was assumed that there is a deterministic control parameter $\Lambda_{o} \equiv \Lambda_{o}(z)$, such that $G_{i j}(z)$ and $Z_{i}(z)$ satisfy $\max _{i, j}\left|G_{i j}\right| \leq C \Lambda_{o}+C \delta_{i j},\left|G_{i i}(z)\right| \geq c$, and $\max _{i}\left|Z_{i}\right| \leq C \Lambda_{o}$, with high probability, and $\Lambda_{o}$ satisfies $\Lambda_{o} \ll 1$, for $\operatorname{Im} z \gg N^{-1}$.

Under the assumption of Lemma 5.9 , the Green function entries $\left(G_{i j}(z)\right)$ can become large, i.e., $\left|G_{i j}(z)\right| \gg 1$, $\operatorname{Im} \eta \sim N^{-1 / 2}$, for certain choices of the spectral parameter $z$ (close to the spectral edge) and certain choice of indices $i, j$. However, resolvent fractions of the form $G_{a b}(z) / G_{b b}(z)$ and $G_{a b}(z) / G_{a a}(z) G_{b b}(z)\left(a, b \geq n_{0}\right)$, are small (see Lemma 6.2 below for a precise statement). Using this observation, we adapt the methods of [17] to control the fluctuation average (6.1).

6.1. Preliminaries. Let $a, b \in \llbracket 1, N \rrbracket$ and $\mathbb{T}, \mathbb{T}^{\prime} \subset \llbracket 1, N \rrbracket$, with $a, b \notin \mathbb{T}, b \notin \mathbb{T}^{\prime}, a \neq b$, then we set

$$
F_{a b}^{\left(\mathbb{T}, \mathbb{T}^{\prime}\right)}(z):=\frac{G_{a b}^{(\mathbb{T})}(z)}{G_{b b}^{\left(\mathbb{T}^{\prime}\right)}(z)}, \quad\left(z \in \mathbb{C}^{+}\right),
$$

and we often abbreviate $F_{a b}^{\left(\mathbb{T}, \mathbb{T}^{\prime}\right)} \equiv F_{a b}^{\left(\mathbb{T}, \mathbb{T}^{\prime}\right)}(z)$. In case $\mathbb{T}=\mathbb{T}^{\prime}=\emptyset$, we simply write $F_{a b} \equiv F_{a b}^{\left(\mathbb{T}, \mathbb{T}^{\prime}\right)}$. Below we will always implicitly assume that $\{a, b\}$ and $\mathbb{T}, \mathbb{T}^{\prime}$ are compatible in the sense that $a \neq b, a, b \notin \mathbb{T}, b \notin \mathbb{T}^{\prime}$.

Starting from (3.10), simple algebra yields the following relations among the $\left\{F_{a b}^{\left(\mathbb{T}, \mathbb{T}^{\prime}\right)}\right\}$.

Lemma 6.1. Let $a, b, c \in \llbracket 1, N \rrbracket$, all distinct, and let $\mathbb{T}, \mathbb{T}^{\prime} \subset \llbracket 1, N \rrbracket$. Then,

(1) for $c \notin \mathbb{T} \cup \mathbb{T}^{\prime}$,

$$
F_{a b}^{\left(\mathbb{T}, \mathbb{T}^{\prime}\right)}=F_{a b}^{\left(\mathbb{T} c, \mathbb{T}^{\prime}\right)}+F_{a c}^{\left(\mathbb{T}, \mathbb{T}^{\prime}\right)} F_{c b}^{\left(\mathbb{T}, \mathbb{T}^{\prime}\right)}
$$

(2) for $c \notin \mathbb{T} \cup \mathbb{T}^{\prime}$,

$$
F_{a b}^{\left(\mathbb{T}, \mathbb{T}^{\prime}\right)}=F_{a b}^{\left(\mathbb{T}, \mathbb{T}^{\prime} c\right)}-F_{a b}^{\left(\mathbb{T}, \mathbb{T}^{\prime} c\right)} F_{b c}^{\left(\mathbb{T}, \mathbb{T}^{\prime}\right)} F_{c b}^{\left(\mathbb{T}, \mathbb{T}^{\prime}\right)}
$$

(3) for $c \notin \mathbb{T}$,

$$
\frac{1}{G_{a a}^{(\mathbb{T})}}=\frac{1}{G_{a a}^{(\mathbb{T} c)}}\left(1-F_{a c}^{(\mathbb{T}, \mathbb{T})} F_{c a}^{(\mathbb{T}, \mathbb{T})}\right)
$$

6.2. The fluctuation average lemma. Recall the definition of the domain $\mathcal{D}_{\epsilon}^{\prime}$ of the spectral parameter in (4.31) and of the constant $\mathfrak{b}>0$ in (4.1). Set $A:=\llbracket n_{0}, N \rrbracket$. To start with, we bound $F_{a b}$ and $F_{a b}^{(\emptyset, a)} / G_{a a}$ on the domain $\mathcal{D}_{\epsilon}^{\prime}$.

Lemma 6.2. Assume that, for all $z \in \mathcal{D}_{\epsilon}^{\prime}$, the estimates

$$
\left|m(z)-\widehat{m}_{f c}(z)\right| \leq N^{-1 / 2+2 \epsilon}, \quad \operatorname{Im} m(z) \leq N^{-1 / 2+2 \epsilon},
$$

hold with $(\xi, \nu)$-high probability on $\Omega_{V}$. 
Then, there exists a constant $c$, such that for all $z \in \mathcal{D}_{\epsilon}^{\prime}$,

$$
\max _{\substack{a, b \in A \\ a \neq b}}\left|F_{a b}(z)\right| \leq\left(\varphi_{N}\right)^{c \xi} N^{-\mathfrak{b} / 2} N^{\epsilon}, \quad\left(z \in \mathcal{D}_{\epsilon}^{\prime}\right),
$$

and

$$
\max _{\substack{a, b \in A \\ a \neq b}}\left|\frac{F_{a b}^{(\emptyset, a)}(z)}{G_{a a}(z)}\right| \leq\left(\varphi_{N}\right)^{c \xi} N^{-1 / 2} N^{2 \epsilon}, \quad\left(z \in \mathcal{D}_{\epsilon}^{\prime}\right),
$$

with $(\xi, \nu)$-high probability on $\Omega_{V}$.

Proof. Dropping the $z$-dependence from the notation, we first note that by Schur's complement formula (3.7) and Inequality (6.8), we have with high probability on $\Omega_{V}$, for $z \in \mathcal{D}_{\epsilon}^{\prime}$,

$$
\frac{1}{G_{a a}^{(b)}}=\lambda v_{a}-z-\widehat{m}_{f c}+\mathcal{O}\left(\left|m-m^{(a b)}\right|\right)+\mathcal{O}\left(N^{-1 / 2+2 \epsilon}\right),
$$

for all $a \in A, b \in \llbracket 1, N \rrbracket, a \neq b$. Thus, for $z \in \mathcal{D}_{\epsilon}^{\prime}$, Lemma 3.3 yields

$$
\left|G_{a a}^{(b)}\right| \leq C\left(\varphi_{N}\right)^{\xi} N^{1 /(\mathrm{b}+1)} N^{\epsilon},
$$

with high probability on $\Omega_{V}$. Further, from the resolvent formula (3.9) we obtain

$$
F_{a b}=-\sum_{k}^{(b)} G_{a k}^{(b)} h_{k b},
$$

for $a, b \in A, a \neq b$. From the large deviation estimate (3.14) we infer that

$$
\left|\sum_{k}^{(b)} G_{a k}^{(b)} h_{k b}\right| \leq\left(\varphi_{N}\right)^{\xi}\left(\frac{\operatorname{Im} G_{a a}^{(b)}}{N \eta}\right)^{1 / 2},
$$

with high probability, and hence conclude by (6.12) that

$$
\left|F_{a b}\right| \leq C\left(\varphi_{N}\right)^{2 \xi} N^{-\mathfrak{b} / 2} N^{\epsilon},
$$

with high probability on $\Omega_{V}$.

To prove the second claim, we recall that, for $a \neq b$, the resolvent formula (3.8) gives

$$
\frac{F_{a b}^{(\emptyset, a)}}{G_{a a}}=-h_{a b}+\sum_{k, l}^{(a b)} h_{a k} G_{k l}^{(a b)} h_{l b},
$$

and we conclude from the large deviation estimates (3.17) and (3.18) that

$$
\left|\frac{F_{a b}^{(\emptyset, a)}}{G_{a a}}\right| \leq \frac{\left(\varphi_{N}\right)^{\xi}}{\sqrt{N}}+\left(\varphi_{N}\right)^{\xi} \sqrt{\frac{\operatorname{Im} m^{(a b)}}{N \eta}},
$$

with high probability. Since $\left|m-m^{(a b)}\right| \leq C N^{-1 / 2+\epsilon}$ on $\mathcal{D}_{\epsilon}^{\prime}$, by Lemma 3.3, we obtain using (6.8)

$$
\left|\frac{F_{a b}^{(\emptyset, a)}}{G_{a a}}\right| \leq\left(\varphi_{N}\right)^{c \xi} N^{-1 / 2} N^{2 \epsilon}
$$

with high probability on $\Omega_{V}$.

Definition 6.3. Let $\Xi$ be an event defined by requiring that the following holds on it: (1) there exists a constant c, such that for all $z \in \mathcal{D}_{\epsilon}^{\prime}$, (6.8), (6.9) and (6.10) hold; (2) there exists a constant $c$ such that, for all $z \in \mathcal{D}_{\epsilon}^{\prime}$ and $a \in A$,

$$
\left|Q_{a}\left(\frac{1}{G_{a a}}\right)\right| \leq\left(\varphi_{N}\right)^{c \xi} N^{-1 / 2+2 \epsilon}
$$

and (3), for all $i, j \in \llbracket 1, N \rrbracket$,

$$
\max _{i j}\left|w_{i j}\right| \leq \frac{\left(\varphi_{N}\right)^{c \xi}}{\sqrt{N}}
$$


By Lemma 6.2. Lemma 5.7. Corollary 5.5, Lemma 5.4 and Inequality (3.18), we know that $\Xi$ holds with $(\xi, \nu)$ high probability on $\Omega_{V}$.

Corollary 6.4. For $p \leq(\log N)^{\xi}$, there exists a constant $c$, such that the following holds. For all $\mathbb{T}, \mathbb{T}^{\prime}, \mathbb{T}^{\prime \prime} \subset A$, with $|\mathbb{T}|,\left|\mathbb{T}^{\prime}\right|,\left|\mathbb{T}^{\prime \prime}\right| \leq p$, for all $a, b \in A, a \neq b$, and, for all $z \in \mathcal{D}_{\epsilon}^{\prime}$, we have

$$
\begin{array}{r}
\mathbb{1}(\Xi)\left|F_{a b}^{\left(\mathbb{T}, \mathbb{T}^{\prime}\right)}(z)\right| \leq\left(\varphi_{N}\right)^{c \xi} N^{-\mathfrak{b} / 2} N^{\epsilon}, \\
\mathbb{1}(\Xi)\left|\frac{F_{a b}^{\left(\mathbb{T}^{\prime}, \mathbb{T}^{\prime \prime}\right)}(z)}{G_{a a}^{(\mathbb{T})}(z)}\right| \leq\left(\varphi_{N}\right)^{c \xi} N^{-1 / 2} N^{2 \epsilon},
\end{array}
$$

and

$$
\mathbb{1}(\Xi)\left|Q_{a}\left(\frac{1}{G_{a a}^{(\mathbb{T})}}\right)\right| \leq\left(\varphi_{N}\right)^{c \xi} N^{-1 / 2} N^{2 \epsilon}
$$

on $\Omega_{V}$, for $N$ sufficiently large.

The proof of this corollary is given in Appendix B.

Before we state the next lemma, we remark that in this section we use the symbol $\mathbb{E}^{W}$ for the partial expectation with respect to the random variables $\left(w_{i j}\right)$ with $\left(v_{i}\right)$ kept fixed, i.e., $\mathbb{E}^{W}[\cdot] \equiv \mathbb{E}\left[\cdot \mid\left(v_{i}\right)\right]$.

Lemma 6.5. Let $p \in \mathbb{N}$ satisfy $p \leq(\log N)^{\xi-3 / 2}$. Let $q \in \llbracket 0, p \rrbracket$ and consider random variables $\left(\mathcal{X}_{i}\right) \equiv\left(\mathcal{X}_{i}(H)\right)$ and $\left(\mathcal{Y}_{i}\right) \equiv\left(\mathcal{Y}_{i}(H)\right), i \in \llbracket 1, p \rrbracket$, satisfying

$$
\mathbb{1}(\Xi)\left|\mathcal{X}_{i}\right| \leq C\left(\varphi_{N}\right)^{c \xi d_{i}} N^{-1 / 2+2 \epsilon} N^{-\left(d_{i}-1\right)(\mathfrak{b} / 2-\epsilon)}, \quad \mathbb{1}(\Xi)\left|Q_{i} \mathcal{Y}_{i}\right| \leq C\left(\varphi_{N}\right)^{c \xi} N^{-1 / 2} N^{2 \epsilon},
$$

where $d_{i} \in \mathbb{N}_{0}$ satisfy $0 \leq s=\sum_{i=1}^{q}\left(d_{i}-1\right) \leq p+2$. Assume moreover that there are constants $C^{\prime}$ and $K$, such that

$$
\mathbb{E}^{W}\left|\mathcal{X}_{i}\right|^{r} \leq\left(C^{\prime} N\right)^{K\left(d_{i}+1\right) r}, \quad \mathbb{E}^{W}\left|\mathcal{Y}_{i}\right|^{r} \leq\left(C^{\prime} N\right)^{K r}
$$

for any $r \in \mathbb{N}$, with $r \leq 10$. Finally, assume that the event $\Xi$ holds with $(\xi, \nu)$-high probability.

Then, there is a constant $c_{0}$, depending only on $C, C^{\prime}, c, K$ in (6.24) and (6.25), and on $\xi$ and $\nu$, such that

$$
\left|\mathbb{E}^{W} \prod_{i=1}^{q} Q_{i}\left(\mathcal{X}_{i}\right) \prod_{i=q+1}^{p} Q_{i}\left(\mathcal{Y}_{i}\right)\right| \leq\left(\varphi_{N}\right)^{c_{0} \xi p} N^{-p / 2-s \mathfrak{b} / 2} N^{(2 p+s) \epsilon}
$$

(Here, we use the convention that, for $q=0$, the first product is set to one, and, similarly, for $q=p$, the second product is set to one.)

Lemma 6.5 is proved in Appendix B.

Next, we state the main result of this section:

Lemma 6.6. [Fluctuation Average Lemma] Let $A:=\llbracket n_{0}, N \rrbracket$. Recall the definition of the domain $\mathcal{D}_{\epsilon}^{\prime}$ in (4.31). Let $\Xi$ denote the event in Definition 6.3 and assume it has $(\xi, \nu)$-high probability. Then there exist constants $C$, $c$, $c_{0}$, such that for $p=2 r, r \in \mathbb{N}, p \leq(\log N)^{\xi-3 / 2}$, we have

$$
\mathbb{E}^{W}\left|\frac{1}{N} \sum_{a \in A} Q_{a}\left(\frac{1}{G_{a a}(z)}\right)\right|^{p} \leq(C p)^{c p}\left(\varphi_{N}\right)^{c_{0} p \xi} N^{-p / 2-p \mathfrak{b} / 2} N^{3 p \epsilon},
$$

for all $z \in \mathcal{D}_{\epsilon}^{\prime}$, on $\Omega_{V}$.

Proof. Fix $z \in \mathcal{D}_{\epsilon}^{\prime}$. For simplicity we drop the $z$-dependence from our notation and we always work on $\Omega_{V}$. We explain the idea of the proof for the simple case $p=2$. First, we note that

$$
\frac{1}{N^{2}} \sum_{a \in A} \mathbb{E}^{W}\left|Q_{a}\left(\frac{1}{G_{a a}}\right)\right|^{2} \leq C\left(\varphi_{N}\right)^{2 c_{0}} \frac{N^{4 \epsilon}}{N^{2}},
$$

where we used (6.26) (with $p=2, q=0, \mathcal{Y}_{1}=\left(\overline{G_{a a}}\right)^{-1}, \mathcal{Y}_{2}=\left(G_{a a}\right)^{-1}$ ). Here, we also used that $\mathbb{E}^{W}\left|G_{a a}\right|^{-r} \leq$ $(C N)^{K r}$, for some $K>0, r \in \mathbb{N}$, (see Remark 6.10 below) to ensure (6.25). It thus suffices to consider

$$
\frac{1}{N^{2}} \sum_{\substack{a_{1} \in A, a_{2} \in A \\ a_{1} \neq a_{2}}} \mathbb{E}^{W} Q_{a_{1}} \overline{\left(\frac{1}{G_{a_{1} a_{1}}}\right)} Q_{a_{2}}\left(\frac{1}{G_{a_{2} a_{2}}}\right) \text {. }
$$


Applying the formula

$$
\frac{1}{G_{a a}}=\frac{1}{G_{a a}^{(b)}}\left(1-F_{a b} F_{b a}\right), \quad(a \neq b),
$$

twice, we obtain

$$
\begin{aligned}
\mathbb{E}^{W} Q_{a_{1}} \overline{\left(\frac{1}{G_{a_{1} a_{1}}}\right)} Q_{a_{2}}\left(\frac{1}{G_{a_{2} a_{2}}}\right) & \left.=\mathbb{E}^{W} Q_{a_{1}} \overline{\left(\frac{1}{G_{a_{1} a_{1}}^{\left(a_{2}\right)}}\left(1-F_{a_{1} a_{2}} F_{a_{2} a_{1}}\right)\right.}\right) Q_{a_{2}}\left(\frac{1}{G_{a_{2} a_{2}}^{\left(a_{1}\right)}}\left(1-F_{a_{2} a_{1}} F_{a_{1} a_{2}}\right)\right) \\
& =\mathbb{E}^{W} Q_{a_{1}} \overline{\left(\frac{1}{G_{a_{1} a_{1}}^{\left(a_{2}\right)}} F_{a_{1} a_{2}} F_{a_{2} a_{1}}\right)} Q_{a_{2}}\left(\frac{1}{G_{a_{2} a_{2}}^{\left(a_{1}\right)}} F_{a_{2} a_{1}} F_{a_{1} a_{2}}\right),
\end{aligned}
$$

where we used that $G_{a a}^{(b)}, a \neq b$, is independent of the entries in the $b$-th column/row of $W$, and that, for general random variables $A=A(W)$ and $B=B(W), \mathbb{E}^{W}\left[\left(Q_{b} A\right) B\right]=\mathbb{E}^{W}\left[B \mathbb{E}_{b} Q_{b} A\right]=0$ if $B$ is independent of the variables in the $b$-th column/row of $W$.

By Corollary 6.4, we have

$$
\mathbb{1}(\Xi)\left|\frac{F_{a_{2} a_{1}}}{G_{a_{1} a_{1}}^{\left(a_{2}\right)}} F_{a_{1} a_{2}}\right| \leq C N^{-1 / 2-\mathfrak{b} / 2} N^{3 \epsilon},
$$

with high probability, and a similar bound holds for the second term in (6.31). Since

$$
\mathbb{E}^{W}\left|\frac{F_{a_{2} a_{1}}}{G_{a_{1} a_{1}}^{\left(a_{2}\right)}} F_{a_{1} a_{2}}\right|^{r} \leq(C N)^{4 K r}
$$

for $r \leq 10 p$, for some $K$, as can be easily checked (c.f., Remark 6.10 below), Lemma 6.5 implies that

$$
\left|\frac{1}{N^{2}} \sum_{a_{1} \in A, a_{2} \in A} \mathbb{E}^{W} Q_{a_{1}} \overline{\left(\frac{1}{G_{a_{1} a_{1}}}\right)} Q_{a_{2}}\left(\frac{1}{G_{a_{2} a_{2}}}\right)\right| \leq C\left(\varphi_{N}\right)^{2 c_{0} \xi} N^{-1-\mathfrak{b}} N^{6 \epsilon},
$$

and we obtain the claim in the sense of second moment bounds.

To deal with higher moments, we abbreviate $\mathbf{a} \equiv\left(a_{1}, \ldots, a_{p}\right)$ and write, for $p=2 r$ satisfying $p \leq(\log N)^{\xi-3 / 2}$,

$$
\begin{aligned}
\mathbb{E}^{W}\left|\frac{1}{N} \sum_{a \in A} Q_{a}\left(\frac{1}{G_{a a}}\right)\right|^{2 r} & =\frac{1}{N^{2 r}} \sum_{a_{1} \in A, \ldots, a_{2 r} \in A} \mathbb{E}^{W} \bar{X}_{a_{1}} \cdots \bar{X}_{a_{r}} X_{a_{r+1}} \cdots X_{a_{2 r}} \\
& =\frac{1}{N^{2 r}} \sum_{a_{1} \in A, \ldots, a_{2 r} \in A} \mathbb{E}^{W} X_{\mathbf{a}}
\end{aligned}
$$

where $X_{a}:=Q_{a}\left(G_{a a}\right)^{-1}$ and $X_{\mathbf{a}}:=\bar{X}_{a_{1}} \cdots \bar{X}_{a_{r}} X_{a_{r+1}} \cdots X_{a_{2 r}}$.

To cope with (6.35) efficiently, we need some more notation. Let $\mathbf{L}, \mathbf{U} \subset \llbracket 1, N \rrbracket$. We denote by $\mathcal{F}(\mathbf{L}, \mathbf{U})$ the set of all "off-diagonal resolvent fractions" $F_{a b}^{\left(\mathbb{T}, \mathbb{T}^{\prime}\right)}, a \neq b, a, b \in \mathbf{L}$ and $\mathbb{T}, \mathbb{T}^{\prime} \subset \mathbf{U}$, with $a, b \notin \mathbb{T}, b \notin \mathbb{T}^{\prime}$. Further, we denote by $\mathcal{G}(\mathbf{L}, \mathbf{U})$ the set of "diagonal resolvent entries" having lower indices in $\mathbf{L}$ and upper indices in $\mathbf{U}$; more precisely, $\mathcal{G}(\mathbf{L}, \mathbf{U}):=\left\{G_{a a}^{(\mathbb{T})}: a \in \mathbf{L}, \mathbb{T} \subset \mathbf{U}, a \notin \mathbb{T}\right\}$. Finally, we denote by $\widetilde{\mathcal{F}}(\mathbf{L}, \mathbf{U})$ the set of monomials of the form $\mathbb{C}_{d}^{-1} F_{1} F_{2} \ldots F_{n}$, with $G_{d} \in \mathcal{G}(\mathbf{L}, \mathbf{U}), F_{i} \in \mathcal{F}(\mathbf{L}, \mathbf{U}), n \in \mathbb{N}_{0}$.

Following [17, we call $F_{a b}^{\left(\mathbb{T}, \mathbb{T}^{\prime}\right)} \in \mathcal{F}(\mathbf{L}, \mathbf{U})$, maximally expanded (in $\mathbf{U}$ ), if $\{a, b\} \cup \mathbb{T} \supset \mathbf{L}$ and $\{b\} \cup \mathbb{T}^{\prime} \supset \mathbf{L}$. Similarly, $G_{a a}^{(\mathbb{T})}$, is maximally expanded if $\{a\} \cup \mathbb{T} \supset \mathbf{L}$, and we call a monomial in $\widetilde{\mathcal{F}}(\mathbf{L}, \mathbf{U})$ maximally expanded if all its factors are maximally expanded. The degree of $F \in \widetilde{\mathcal{F}}(\mathbf{L}, \mathbf{U})$ is defined as the number of factors of $F_{a b}^{\left(\mathbb{T}, \mathbb{T}^{\prime}\right)}$ in $F($ with $a \neq b)$.

Next, we define a recursive procedure that, given $F \in \widetilde{\mathcal{F}}(\mathbf{L}, \mathbf{U})$, successively adds upper indices from the set $\mathbf{U}$ to $F$, at the expense of generating terms of higher degree. This procedure is iterated until either all generated terms are maximally expanded or their degree is sufficiently large, so that they can be neglected.

Given $F \in \widetilde{\mathcal{F}}(\mathbf{L}, \mathbf{U})$ the recursive procedure is as follows:

(A) Stopping rules: If the degree of $F$ is bigger equal $p+1$ or if $F$ is maximally expanded in $\mathbf{U}$, we stop the procedure. 
(B) Iteration: Else, we choose an arbitrary non-maximally expanded factor $F_{a b}^{\left(\mathbb{T}, \mathbb{T}^{\prime}\right)}$ or $1 / G_{a a}^{(\mathbb{T})}$ of $F$ and split it using, for the former choice, either

$$
F_{a b}^{\left(\mathbb{T}, \mathbb{T}^{\prime}\right)}=F_{a b}^{\left(\mathbb{T} c, \mathbb{T}^{\prime}\right)}+F_{a c}^{\left(\mathbb{T}, \mathbb{T}^{\prime}\right)} F_{c b}^{\left(\mathbb{T}, \mathbb{T}^{\prime}\right)}
$$

for the smallest $c \in \mathbf{U} \backslash(\mathbb{T} a b)$, or

$$
F_{a b}^{\left(\mathbb{T}, \mathbb{T}^{\prime}\right)}=F_{a b}^{\left(\mathbb{T}, \mathbb{T}^{\prime} c\right)}-F_{a b}^{\left(\mathbb{T}, \mathbb{T}^{\prime} c\right)} F_{b c}^{\left(\mathbb{T}, \mathbb{T}^{\prime}\right)} F_{c b}^{\left(\mathbb{T}, \mathbb{T}^{\prime}\right)},
$$

for the smallest $c \in \mathbf{U} \backslash\left(\mathbb{T}^{\prime} b\right)$, respectively,

$$
\frac{1}{G_{a a}^{(\mathbb{T})}}=\frac{1}{G_{a a}^{(\mathbb{T} c)}}\left(1-F_{a c}^{(\mathbb{T}, \mathbb{T})} F_{c a}^{(\mathbb{T}, \mathbb{T})}\right)
$$

for the smallest $c \in \mathbf{U} \backslash(\mathbb{T} a)$, for the latter choice.

Then, we start over with $F$ being one of the generated monomials.

We remark that a similar algorithm (expanding the resolvent entries $G_{i j}^{(\mathbb{T})}$ instead of $F_{i j}^{\left(\mathbb{T}, \mathbb{T}^{\prime}\right)}$ ) appeared first in 18$]$; see also [17.

Remark 6.7. This recursive procedure contains some arbitrariness, e.g., in the specific choice of $F_{a b}^{\left(\mathbb{T}, \mathbb{T}^{\prime}\right)}$ in (6.36), (6.37) or (6.38). This arbitrariness does not affect the argument and we thus choose not to remove it.

We now use the above algorithm to expand the right side of (6.35): Denote by $\mathcal{P}_{2 r}$ the set of partitions of $\llbracket 1,2 r \rrbracket$ and let $\Gamma(\mathbf{a}) \in \mathcal{P}_{2 r}$ be the partition induced by the equivalence relation $i \sim j$, if and only if $a_{i}=a_{j}, i, j \in \llbracket 1,2 r \rrbracket$. Then we can write

$$
\mathbb{E}^{W}\left|\frac{1}{N} \sum_{a \in A} Q_{a}\left(\frac{1}{G_{a a}}\right)\right|^{2 r}=\frac{1}{N^{2 r}} \sum_{\Gamma \in \mathcal{P}_{2 r}} \sum_{a_{1} \in A, \ldots, a_{2 r} \in A} \mathbb{1}(\Gamma=\Gamma(\mathbf{a})) \mathbb{E}^{W} X_{\mathbf{a}} .
$$

Given $\mathbf{a}=\left(a_{i}\right)$, denote by $\Gamma:=\Gamma(\mathbf{a})$, the partition induced by the equivalence relation $\sim$. For a label $i \in \llbracket 1,2 r \rrbracket$, we denote by $[i]$ the block of $i$ in $\Gamma$. Let $S(\Gamma):=\{i:|[i]|=1\} \subset \llbracket 1,2 r \rrbracket$ denote the set of singletons or single labels and abbreviate by $s:=|S(\Gamma)|$ its cardinality. We denote by $\mathbf{A}_{S} \equiv \mathbf{A}_{S(\Gamma)}:=\left\{a_{i}\right\}_{i \in S}$, the summation indices associated with single labels. Notice that if $i$ is a single label (for some $\Gamma$ ), then there is exactly one $Q_{a_{i}}$ on the right side of (6.39). However, if $i$ is not a single label (for some $\Gamma$ ), $Q_{a_{i}}$ appears more than once on the right side of (6.39).

We now choose $\mathbf{L}=\mathbf{A}_{\Gamma} \equiv\left\{a_{i}\right\}_{i}$ and $\mathbf{U}=\mathbf{A}_{S(\Gamma)} \equiv \mathbf{A}_{S}$ and apply the algorithm $(A)-(B)$ to $X_{a_{i}}$ to obtain

$$
X_{a_{i}}=X_{a_{i}}^{\left(\mathbf{A}_{S} \backslash a_{i}\right)}+Q_{a_{i}} M_{a_{i}}\left(\mathbf{A}_{\Gamma}, \mathbf{A}_{S}\right)+Q_{a_{i}} R_{a_{i}}\left(\mathbf{A}_{\Gamma}, \mathbf{A}_{S}\right)
$$

where $X_{a_{i}}^{\left(\mathbf{A}_{S} \backslash a_{i}\right)}:=Q_{a_{i}}\left(G_{a_{i} a_{i}}^{\left(\mathbf{A}_{S} \backslash a_{i}\right)}\right)^{-1}$, and where $M_{a_{i}}\left(\mathbf{A}_{\Gamma}, \mathbf{A}_{S}\right)$ and $R_{a_{i}}\left(\mathbf{A}_{\Gamma}, \mathbf{A}_{S}\right)$ are sums of elements in $\widetilde{\mathcal{F}}\left(\mathbf{A}_{\Gamma}, \mathbf{A}_{S}\right)$, such that each term in $M_{a_{i}}\left(\mathbf{A}_{\Gamma}, \mathbf{A}_{S}\right)$ is maximally expanded in $\mathbf{A}_{S}$ and each summand in $R_{a_{i}}\left(\mathbf{A}_{\Gamma}, \mathbf{A}_{S}\right)$ is of degree $p+1$ or higher.

Remark 6.8. The stopping rules ensure that the procedure stops after a finite number of steps when applied to a $X_{a_{i}}$. More precisely, one checks that the number of terms on the ride side of (6.40) is bounded by $(C p)^{c p}$, for some constants $C, c$; see, e.g., [17], page 54 .

Remark 6.9. Ignoring for the moment the upper indices, we observe that each summand in $M_{a_{i}}\left(\mathbf{A}_{\Gamma}, \mathbf{A}_{S}\right)$ or $R_{a_{i}}\left(\mathbf{A}_{\Gamma}, \mathbf{A}_{S}\right)$ is of the form

$$
\frac{F_{a_{i} b_{1}}^{\#}}{G_{a_{i} a_{i}}^{\#}} \cdot F_{b_{1} b_{2}}^{\#} F_{b_{2} b_{3}}^{\#} \cdots F_{b_{n} a_{i}}^{\#}
$$

for some $\left(b_{1}, \ldots, b_{n}\right) \in \mathbf{A}_{S}^{n}$, with $1 \leq n \leq p+1$, satisfying $b_{1} \neq a_{i}, b_{k} \neq b_{k+1},(k \in \llbracket 1, n-1 \rrbracket), b_{n} \neq a_{1}$. Here \# stands for some appropriate $\left(\mathbb{T}, \mathbb{T}^{\prime}\right)$, with $|\mathbb{T}| \leq p-2,\left|\mathbb{T}^{\prime}\right| \leq p-1$. Indeed, when applying the recursive procedure to $1 / G_{a_{i} a_{i}}$, the first step yields

$$
\frac{1}{G_{a_{i} a_{i}}}=\frac{1}{G_{a_{i} a_{i}}^{\left(b_{1}\right)}}\left(1-F_{a_{i} b_{1}} F_{b_{1} a_{i}}\right)
$$

for some $b_{1} \in \mathbf{A}_{S} \backslash\left\{a_{i}\right\}$, which is of the claimed form. Applying (6.36), (6.37) or (6.38) to a factor in (6.42) yields again two terms of the form (6.41), etc. The claim then follows by noticing that the stopping rules ensure that each summand is of degree less equal $p+2$. 
Using (6.21) $n$ times and (6.22) once, we obtain

$$
\mathbb{1}(\Xi)\left|\frac{F_{a_{i} b_{1}}^{\#}}{G_{a_{i} a_{i}}^{\#}} \cdot F_{b_{1} b_{2}}^{\#} F_{b_{2} b_{3}}^{\#} \cdots F_{b_{n} a_{i}}^{\#}\right| \leq C^{n+1}\left(\varphi_{N}\right)^{c \xi(n+1)} N^{-1 / 2} N^{-n \mathfrak{b} / 2} N^{(n+2) \epsilon},
$$

irrespective of the particular choice of the upper indices (recall that \# stands for some $\left(\mathbb{T}, \mathbb{T}^{\prime}\right)$ satisfying $|\mathbb{T}|,\left|\mathbb{T}^{\prime}\right| \leq$ $\left.(\log N)^{\xi}\right)$. For $n=0$, the analogous bound to (6.43) reads

$$
\mathbb{1}(\Xi)\left|Q_{a_{i}}\left(\frac{1}{G_{a_{i} a_{i}}^{\#}}\right)\right| \leq C\left(\varphi_{N}\right)^{c \xi} N^{-1 / 2} N^{2 \epsilon}
$$

see $(6.23)$.

Remark 6.10. Still ignoring the upper indices, we remark that in order to apply Lemma 6.5. we need to check that

$$
\mathbb{E}^{W}\left|\frac{F_{a_{i} b_{1}}^{\#}}{G_{a_{i} a_{i}}^{\#}} \cdot F_{b_{1} b_{2}}^{\#} F_{b_{2} b_{3}}^{\#} \cdots F_{b_{n} a_{i}}^{\#}\right|^{r} \leq(C N)^{K r(n+1)},
$$

for some constants $C$ and $K$, for all $r \leq 10 p$. Starting from Schur's formula

$$
\frac{1}{G_{a a}^{(\mathbb{T})}}=\lambda v_{a}+w_{a a}-z-\sum_{k, l}^{(\mathbb{T} a)} w_{a k} G_{k l}^{(a \mathbb{T})} w_{l a}, \quad(a \notin \mathbb{T})
$$

and the trivial bounds $\left|G_{a a}^{(\mathbb{T})}\right| \leq \eta^{-1} \leq N, \mathbb{E}^{W}\left|w_{i j}\right|^{q} \leq C(\theta q)^{\theta q} N^{-q / 2}$ and $\left|\lambda v_{i}\right|^{q} \leq C^{q}$, and the boundedness of $\mathcal{D}_{\epsilon}^{\prime}$, one checks 6.45) by inspection.

Combining Remarks 6.8 and 6.9 we obtain a bound on the remainder term $R_{a_{i}}\left(\mathbf{A}_{\Gamma}, \mathbf{A}_{S}\right)$,

$$
\mathbb{1}(\Xi)\left|R_{a_{i}}\left(\mathbf{A}_{\Gamma}, \mathbf{A}_{S}\right)\right| \leq(C p)^{2 p}\left(\varphi_{N}\right)^{c \xi p} N^{-1 / 2} N^{-p \mathfrak{b} / 2} N^{(p+2) \epsilon} .
$$

To condense the notation slightly, we abbreviate $M_{a_{i}} \equiv M_{a_{i}}\left(\mathbf{A}_{\Gamma}, \mathbf{A}_{S}\right), R_{a_{i}} \equiv R_{a_{i}}\left(\mathbf{A}_{\Gamma}, \mathbf{A}_{S}\right)$ and set

$$
M_{a_{i}}^{\prime}:=\left(G_{a_{i} a_{i}}^{\left(\mathbf{A}_{S} \backslash a_{i}\right)}\right)^{-1}+M_{a_{i}}
$$

Returning to (6.39) and choosing $i=1$, we can write

$$
\mathbb{E}^{W} X_{\mathbf{a}}=\mathbb{E}^{W} Q_{a_{1}}\left(\bar{M}_{a_{1}}^{\prime}\right) \bar{X}_{a_{2}} \cdots X_{a_{2 r}}+\mathcal{R}_{a_{1}}
$$

where we have set $\mathcal{R}_{a_{1}}:=\mathbb{E}^{W} Q_{a_{1}}\left(\bar{R}_{a_{1}}\right) \bar{X}_{a_{2}} \cdots X_{a_{p}}$. By the bound (6.46) and the bounds in Remark 6.10, Lemma 6.5 yields

$$
\left|\mathcal{R}_{a_{1}}\right| \leq(C p)^{c p}\left(\varphi_{N}\right)^{c_{0} \xi p} N^{-p / 2-p \mathfrak{b} / 2+3 p \epsilon}
$$

Before we expand in a next step $X_{a_{2}}$ in (6.39), we make the following observation.

Remark 6.11. Let $F \in \widetilde{F}\left(\mathbf{A}_{\Gamma}, \mathbf{A}_{S}\right)$ be a maximally expanded monomial of degree $d=n+1$. From Remark 6.9, we know that $F$ is of the form (6.41). Since $F$ is maximally expanded, the lower indices in each factor in (6.41) also determine its upper indices. Thus, a summand $F \in \widetilde{\mathcal{F}}\left(\mathbf{A}_{\Gamma}, \mathbf{A}_{S}\right)$ in $M_{a_{i}}$ can be labeled by a sequence $\mathbf{b}=$ $\left(b_{1}, \ldots, b_{n}\right) \in \mathbf{A}_{S}^{n}$, satisfying $b_{1} \neq a_{i}, b_{k} \neq b_{k+1},(k \in \llbracket 1, n-1 \rrbracket), b_{n} \neq a_{i}$. Here $d=n+1, n \geq 1$, is the degree of $F$. In the following we write $F \equiv F_{a_{i}, \mathbf{b}} \in \widetilde{\mathcal{F}}\left(\mathbf{A}_{\Gamma}, \mathbf{A}_{S}\right)$. Denoting by $\mathbf{B}\left(a_{i}, n\right)$ the set of such labeling sequences of length $n$, we can write

$$
M_{a_{i}}=\sum_{n=1}^{p} \sum_{\mathbf{b} \in \mathbf{C}\left(a_{i}, n\right)} F_{a_{i}, \mathbf{b}}
$$

where $\mathbf{C}\left(a_{i}, n\right)$ is some subset of $\mathbf{B}\left(a_{i}, n\right)$. For later purposes, we note the following crude upper bound on the cardinality of $\mathbf{B}\left(a_{i}, n\right)$ :

$$
\left|\mathbf{B}\left(a_{i}, n\right)\right| \leq\left|\mathbf{A}_{S}\right|^{n} \leq p^{n} \leq p^{p}, \quad(n \geq 1) .
$$

For $n=0$, we set $F_{a_{i},(0)}:=\left(G_{a_{i} a_{i}}^{\left(\mathbf{A}_{S} \backslash a_{i}\right)}\right)^{-1}$ and $\mathbf{B}\left(a_{i}, 0\right):=\{(0)\}$. 
Using Remark 6.11, we can write, for $X_{\mathbf{a}}$ as in (6.39),

$$
\mathbb{E}^{W} X_{\mathbf{a}}=\sum_{n_{1}=0}^{p} \sum_{\mathbf{b}_{1} \in \mathbf{C}\left(a_{1}, n_{1}\right)} \mathbb{E}^{W} Q_{a_{1}}\left(\bar{F}_{a_{1}, \mathbf{b}_{1}}\right) \bar{X}_{a_{2}} \cdots X_{a_{p}}+\mathcal{R}_{a_{1}}
$$

where the $n_{1}=0$ term in the sum is understood as $\mathbb{E}^{W} Q_{a_{1}}\left(\bar{F}_{a_{1},(0)}\right) \bar{X}_{a_{2}} \cdots X_{a_{p}}$.

Next, we expand $X_{a_{2}}$ in each summand

$$
\mathbb{E}^{W} Q_{a_{1}}\left(\bar{F}_{a_{1}, \mathbf{b}_{1}}\right) \bar{X}_{a_{2}} \cdots X_{a_{p}}
$$

using the algorithm $(A)-(B)$, however, we stop expanding a term (generated from $X_{a_{2}}$ ), if its degree is bigger than $p+1-n_{1}$, or if it is maximally expanded. Note that we do not expand the rest term $\mathcal{R}_{a_{1}}$ any further. With this modified stopping rule, we arrive at

$$
\begin{gathered}
\mathbb{E}^{W} X_{\mathbf{a}}=\sum_{n_{1}=0}^{p} \sum_{n_{2}=0}^{p} \sum_{\mathbf{b}_{\mathbf{1}} \in \mathbf{C}\left(a_{1}, n_{1}\right)} \sum_{\mathbf{b}_{\mathbf{2}} \in \mathbf{C}\left(a_{2}, n_{2}\right)} \mathbb{1}\left(n_{1}+n_{2} \leq p\right) \mathbb{E}^{W} Q_{a_{1}}\left(\bar{F}_{a_{1}, \mathbf{b}_{1}}\right) Q_{a_{2}}\left(\bar{F}_{a_{2}, \mathbf{b}_{2}}\right) \cdots X_{a_{p}} \\
+\mathcal{R}_{a_{1}}+\mathcal{R}_{a_{2}}
\end{gathered}
$$

for some sets of labeling sequences $\mathbf{C}\left(a_{1}, n_{1}\right)$ and $\mathbf{C}\left(a_{2}, n_{2}\right)$. It is easy to check that the rest term $\mathcal{R}_{a_{2}}$ satisfies the same bound (with possibly slightly larger constants) as $R_{a_{1}}$. This is checked in the same way as before. To estimate the number of summands in $R_{a_{2}}$, we note that we apply the algorithm $\left|\mathbf{C}\left(a_{1}, n_{1}\right)\right|$ times and each application yields no more than $(C p)^{c p}$ terms; see Remark 6.8. Thus the number of summands in $\mathcal{R}_{a_{2}}$ is bounded by $(C p)^{c^{\prime} p}, c^{\prime}>c$.

We continue expanding the remaining $X_{a_{k}}, k \geq 3$, in (6.54) using the algorithm $(A)-(B)$, but while expanding $X_{a_{k}}$, we stop the expansion as soon as the degree of a generated term exceeds $p+1-\sum_{i=1}^{k-1} n_{i}$. This leads to,

$$
\mathbb{E}^{W} X_{\mathbf{a}}=\sum_{n_{1}, \ldots, n_{p}=0}^{p} \sum_{\mathbf{b}_{\mathbf{1}} \in \mathbf{C}\left(a_{1}, n_{1}\right)} \ldots \sum_{\mathbf{b}_{\mathbf{p}} \in \mathbf{C}\left(a_{p}, n_{p}\right)} \mathbb{1}\left(\sum_{i=1}^{p} n_{i} \leq p\right) \mathbb{E}^{W} Y\left(\mathbf{a}, \mathbf{b}_{\mathbf{1}}, \ldots, \mathbf{b}_{\mathbf{p}}\right)+\sum_{i=1}^{p} \mathcal{R}_{a_{i}},
$$

where $\left(\mathbf{C}\left(a_{i}, n_{i}\right)\right)$ are subsets of $\left(\mathbf{B}\left(a_{i}, n_{i}\right)\right)$, and where we have abbreviated

$$
Y\left(\mathbf{a}, \mathbf{b}_{\mathbf{1}}, \ldots, \mathbf{b}_{\mathbf{p}}\right):=\prod_{i=1}^{r} Q_{a_{i}}\left(\bar{F}_{a_{i}, \mathbf{b}_{\mathbf{i}}}\right) \prod_{i=r+1}^{2 r} Q_{a_{i}}\left(F_{a_{i}, \mathbf{b}_{i}}\right) .
$$

The remainder terms $\left(\mathcal{R}_{a_{i}}\right)$ clearly satisfy

$$
\left|\mathcal{R}_{a_{i}}\right| \leq(C p)^{c p}\left(\varphi_{N}\right)^{c_{0} \xi p} N^{-p / 2-p \mathfrak{b} / 2} N^{3 p},
$$

for all $i \in \llbracket 1, p \rrbracket$. It thus suffices to bound the first term on the right side of (6.55).

Following [17, we pick a term $Y \equiv Y\left(\mathbf{a}, \mathbf{b}_{\mathbf{1}}, \ldots, \mathbf{b}_{\mathbf{p}}\right)$ of the form (6.56) that has a non-vanishing expectation, $\mathbb{E}^{W} Y \neq 0$. Considering a single label $i \in S \equiv S(\Gamma)$, we know that there exists a label $j \in S \backslash\{i\}$, such that the monomial $F_{a_{j}, \mathbf{b}_{\mathbf{j}}} \in \widetilde{\mathcal{F}}\left(\mathbf{A}_{\Gamma}, \mathbf{A}_{S}\right)$ in (6.56) contains a factor $F \in \mathcal{F}\left(\mathbf{A}_{\Gamma}, \mathbf{A}_{S}\right)$, having $a_{i}$ as a lower index (otherwise the expectation of $Y$ has to vanish due to the presence of the $Q_{a_{i}}$ ). It follows from Remark 6.9 that $F_{a_{j}, \mathbf{b}_{\mathbf{j}}}$ is of the form (6.41), with $n \geq 1$ and $b_{k}=a_{i}$, for some $k \in \llbracket 1, n \rrbracket$. Note that we use here that all indices in $\mathbf{A}_{S}$ are distinct.

We write $j=\mathfrak{l}(i)$, if a label $j$ is linked to a label $i$ in the sense of the previous paragraph. Denoting by $l_{j}:=\left|\mathfrak{r}^{(-1)}(\{j\})\right|$, the number of times the label $j$ has been chosen to be linked in the above sense to some other label, we obtain

$$
\mathbb{1}(\Xi)\left|F_{a_{j}, \mathbf{b}_{j}}\right| \leq C^{p}\left(\varphi_{N}\right)^{c \xi p} N^{-1 / 2} N^{-l_{j} \mathfrak{b} / 2} N^{2 p \epsilon+l_{j} \epsilon},
$$

as follows from (6.43).

Finally, using $\sum_{j \in S} l_{j} \geq|S|=s$, we get

$$
\mathbb{1}(\Xi)|Y| \leq C^{p}\left(\varphi_{N}\right)^{c \xi p} N^{-p / 2} N^{-s \mathfrak{b} / 2} N^{2 p \epsilon+s \epsilon},
$$

for $Y$ as in (6.56), with $\mathbb{E}^{W} Y \neq 0$. Lemma 6.5 thus gives

$$
\left|\mathbb{E}^{W} Y\right| \leq C^{p}\left(\varphi_{N}\right)^{c_{0} \xi p} N^{-p / 2-s \mathfrak{b} / 2} N^{2 p \epsilon+s \epsilon} .
$$


To bound the right side of (6.55), it remains to bound the number of summands in the first term. Using (6.51), we obtain

$$
\begin{aligned}
\sum_{n_{1}, \ldots, n_{p}=0}^{p} \sum_{\mathbf{b}_{\mathbf{1}} \in \mathbf{C}\left(a_{1}, n_{1}\right)} \ldots \sum_{\mathbf{b}_{\mathbf{p}} \in \mathbf{C}\left(a_{p}, n_{p}\right)} \mathbb{1}\left(\sum_{i=1}^{p} n_{i} \leq p\right) & \leq \sum_{n_{1}, \ldots, n_{p}=0}^{p} \mathbb{1}\left(\sum_{i=1}^{p} n_{i} \leq p\right) \prod_{i=1}^{p} p^{n_{i}} \\
& \leq \sum_{n_{1}, \ldots, n_{p}=0}^{p} p^{p} \leq(C p)^{2 p}
\end{aligned}
$$

and we can bound 6.55

$$
\left|\mathbb{E}^{W} X_{\mathbf{a}}\right| \leq(C p)^{c p}\left(\varphi_{N}\right)^{c_{0} \xi p} N^{-p / 2-s \mathfrak{b} / 2+3 p \epsilon+s \epsilon} .
$$

We now return to (6.39). We perform the summation by first fixing a partition $\Gamma \in \mathcal{P}_{2 r}$. Then we observe that

$$
\frac{1}{N^{2 r}} \sum_{\mathbf{a}} \mathbb{1}(\Gamma=\Gamma(\mathbf{a})) \leq\left(\frac{1}{N}\right)^{2 r-|\Gamma|} \leq\left(\frac{1}{\sqrt{N}}\right)^{2 r-s}
$$

since any block in the partition $\Gamma$ that is not associated to a single label consists of at least two elements. Thus $|\Gamma| \leq(2 r+s) / 2=r+s / 2$. Using $N^{-1 / 2} \ll N^{-\mathfrak{b}}$ we find

$$
\mathbb{E}^{W}\left|\frac{1}{N} \sum_{a \in A} Q_{a}\left(\frac{1}{G_{a a}}\right)\right|^{2 r} \leq(C p)^{c p}\left(\varphi_{N}\right)^{c \xi p} \sum_{\Gamma \in \mathcal{P}_{2 r}} N^{-p / 2-p \mathfrak{b} / 2} N^{3 p \epsilon} .
$$

Recalling that the number of partitions of $p$ elements is bounded by $(C p)^{2 p}$, we thus get

$$
\mathbb{E}^{W}\left|\frac{1}{N} \sum_{a \in A} Q_{a}\left(\frac{1}{G_{a a}(z)}\right)\right|^{2 r} \leq(C p)^{c p}\left(\varphi_{N}\right)^{c_{0} \xi p} N^{-p / 2-p \mathfrak{b} / 2} N^{3 p \epsilon}
$$

for some constants $C$ and $c$. Finally, we note that the constants can be chosen uniformly in $z \in \mathcal{D}_{\epsilon}^{\prime}$, since all estimates used are uniform in $z$.

\section{Next, we prove Lemma 5.9}

Proof of Lemma 5.9. Recalling the remark after Definition 6.3, we know that the event $\Xi$ has $(\xi, \nu)$-high probability. Choosing $p$ as the largest integer smaller than $\nu(\log N)^{\xi-2}$, Markov's inequality and the moment bound (6.27) yield, for some constant $c$,

$$
\frac{1}{N}\left|\sum_{a=n_{0}}^{N} Z_{a}(z)\right| \leq\left(\varphi_{N}\right)^{c \xi} N^{-1 / 2-\mathfrak{b} / 2+4 \epsilon}
$$

with $(\xi-2, \nu)$-high probability on $\Omega_{V}$, uniformly for $z \in \mathcal{D}_{\epsilon}^{\prime}$. This finishes the proof of (5.26).

To prove (5.27), it suffices to replace $\left(G_{a a}\right)$ in (6.2), by $\left(G_{a a}^{(k)}: a \neq k\right)$. Using (6.7) and the bounds (6.21) and (6.22), the claim (5.27), follows from (5.26). We leave the details aside.

Next, we prove Corollary 5.10, Define, for $a \in A$,

$$
g_{a}(z):=\frac{1}{\lambda v_{a}-z-\widehat{m}_{f c}(z)}, \quad\left(z \in \mathbb{C}^{+}\right) .
$$

Note that for $z \in \mathcal{D}_{\epsilon}^{\prime}$, we have $\left|g_{a}(z)\right| \leq C N^{1 /(\mathrm{b}+1)} N^{\epsilon}$ on $\Omega_{V}$.

Lemma 6.12. Let $A=\llbracket n_{0}, N \rrbracket$. Recall the definition of the domain $\mathcal{D}_{\epsilon}^{\prime}$ in (4.31). Let $\Xi$ denote the event in Definition 6.3 and assume it has $(\xi, \nu)$-high probability. Then there exist constants $C, c, c_{0}$, such that for $p=2 r$, $r \in \mathbb{N}, p \leq(\log N)^{\xi-3 / 2}$, we have

$$
\mathbb{E}^{W}\left|\frac{1}{N} \sum_{a \in A}\left(g_{a}(z)\right)^{2} Q_{a}\left(\frac{1}{G_{a a}(z)}\right)\right|^{p} \leq(C p)^{c p}\left(\varphi_{N}\right)^{c_{0} \xi p} N^{-p / 2-p \mathfrak{b} / 2} N^{3 p \epsilon}
$$

on $\Omega_{V}$, for all $z \in \mathcal{D}_{\epsilon}^{\prime}$. 
Proof. First, we note that $g_{a} Q_{b}=Q_{b} g_{a}, a, b \in \llbracket 1, N \rrbracket$, since the $\left(g_{a}\right)$ are independent of the random variables $\left(w_{i j}\right)$. Similar to (6.35), we are led to consider

$$
\mathbb{E}^{W}\left|\frac{1}{N} \sum_{a \in A} g_{a}^{2} Q_{a}\left(\frac{1}{G_{a a}}\right)\right|^{2 r}=\frac{1}{N^{2 r}} \sum_{a_{1} \in A, \ldots, a_{2 r} \in A} g_{\mathbf{a}}^{2} \mathbb{E}^{W} X_{\mathbf{a}}
$$

where we have set $g_{\mathbf{a}}:=\bar{g}_{a_{1}} \cdots \bar{g}_{a_{r}} g_{a_{r+1}} \cdots g_{a_{2 r}}$.

Following the lines of the proof of Lemma 6.6, we write (6.69) as

$$
\mathbb{E}^{W}\left|\frac{1}{N} \sum_{a \in A} g_{a}^{2} Q_{a}\left(\frac{1}{G_{a a}}\right)\right|^{2 r}=\frac{1}{N^{2 r}} \sum_{\Gamma \in \mathcal{P}_{2 r}} \sum_{a_{1} \in A, \ldots, a_{2 r} \in A} \mathbb{1}(\Gamma=\Gamma(\mathbf{a})) g_{\mathbf{a}}^{2} \mathbb{E}^{W} X_{\mathbf{a}}
$$

As in the proof of Lemma 6.6 we fix a and denote by $\Gamma:=\Gamma(\mathbf{a})$, the partition induced by the equivalence relation $\sim$. Since $\mathbb{E}^{W} X_{\mathbf{a}}$ with $\mathbf{a} \equiv \mathbf{a}_{\Gamma}$ has already been bounded in the proof of Lemma 6.6, with a bound that only depends on $s \equiv|S(\Gamma)|($ see $(\underline{6.62})$ ), it suffices to control

$$
\frac{1}{N^{2 r}} \sum_{a_{1} \in A, \ldots, a_{2 r} \in A} \mathbb{1}(\Gamma=\Gamma(\mathbf{a})) g_{\mathbf{a}}^{2} .
$$

Recall that

$$
\frac{1}{N} \sum_{i=1}^{N} \frac{1}{\left|\lambda v_{i}-z-\widehat{m}_{f c}(z)\right|^{2}}=\widehat{R}_{2}(z)<1, \quad\left(z \in \mathcal{D}_{\epsilon}^{\prime}\right)
$$

see, e.g., (4.35). Applying (6.72) $|\Gamma|$ times, we obtain

$$
\frac{1}{N^{2 r}} \sum_{a_{1} \in A, \ldots, a_{2 r} \in A} \mathbb{1}(\Gamma=\Gamma(\mathbf{a}))\left|g_{\mathbf{a}}\right|^{2} \leq C^{2 r}\left(\frac{N^{2 /(\mathrm{b}+1)} N^{2 \epsilon}}{N}\right)^{2 r-|\Gamma|} .
$$

Here $|\Gamma|$ denotes the number of blocks of the partition $\Gamma$, and $s$ denotes the number of single labels in a. Since every block of $\Gamma$ that is not associated with a single label consists of at least two elements, we have $|\Gamma| \leq(2 r+s) / 2=$ $r+s / 2$, thus $2 r-|\Gamma| \geq r-s / 2$, and we obtain,

$$
\begin{aligned}
\frac{1}{N^{2 r}} \sum_{a_{1} \in A, \ldots, a_{2 r} \in A} \mathbb{1}(\Gamma=\Gamma(\mathbf{a}))\left|g_{\mathbf{a}}\right|^{2} & \leq C^{2 r}\left(\frac{N^{2 /(\mathrm{b}+1)} N^{2 \epsilon}}{N}\right)^{r-s / 2} \\
& =C^{2 r}\left(\frac{N^{1 /(\mathrm{b}+1)} N^{\epsilon}}{\sqrt{N}}\right)^{2 r-s} \\
& \leq C^{2 r}\left(N^{-\mathfrak{b}}\right)^{2 r-s} N^{(2 r-s) \epsilon}
\end{aligned}
$$

The proof of Lemma 6.12 is now completed as the proof of Lemma 6.6.

Proof of Corollary 5.10. Recalling (6.2), Corollary 5.10 can be proven in the same way as Lemma 5.9 above.

\section{Proof of Theorem 2.11}

In this section, we prove Theorem 2.11. The proof of (2.21) is based on an analysis of $\left(G_{j j}(z)\right)$, for $z$ close to the eigenvalues $\mu_{k}, k \in \llbracket 1, n_{0}-1 \rrbracket$, using the Helffer-Sjöstrand formula (7.10) below. The Helffer-Sjöstrand calculus has been applied in [19] to $m(z)$, the averaged Green function, respectively to the empirical eigenvalue counting function, to obtain rigidity estimates on the eigenvalue locations. In Subsection 7.2, we apply the Helffer-Sjöstrand formula to $\left(G_{j j}(z)\right)$, respectively, to a weighted empirical eigenvalue counting function; see (7.6).

In Subsection 7.3, we prove (2.22) following the argument given in [23] for generalized Wigner matrices. 
7.1. Preliminaries. To start with, we claim that, for $z$ close to the spectral edge, $z+\widehat{m}_{f c}(z)$ is well approximated by a linear function.

Lemma 7.1. On $\Omega_{V}$, we have for all $z \in \mathcal{D}_{\epsilon}^{\prime}$ that

$$
z+\widehat{m}_{f c}(z)=\lambda-\frac{\lambda^{2}}{\lambda_{+}^{2}-\lambda^{2}}\left(z-L_{+}\right)+\mathcal{O}\left(\left(\varphi_{N}\right)^{\xi}(\kappa+\eta)^{\min \{\mathrm{b}, 2\}}\right)+\mathcal{O}\left(N^{-1 / 2+2 \epsilon}\right) .
$$

Proof. The lemma follows readily by combining Lemma 4.2 and Lemma 4.5

Remark 7.2. Combining Lemma 7.1 and Proposition 4.8, we obtain, for $j \leq n_{0}-1$,

$$
\begin{aligned}
\lambda v_{j}-z-\widehat{m}_{f c}(z)=\frac{\lambda^{2}}{\lambda^{2}-\lambda_{+}^{2}}\left(\mu_{j}-z\right)+\mathcal{O}\left(\left(\varphi_{N}\right)^{2 \xi} N^{-2 /(\mathrm{b}+1)}\right) & \\
& +\mathcal{O}\left(N^{-1 / 2+3 \epsilon}\right)+\mathcal{O}\left(\left(\varphi_{N}\right)^{\xi}(\kappa+\eta)^{\min \{\mathrm{b}, 2\}}\right)
\end{aligned}
$$

with $(\xi, \nu)$-high probability, for all $z \in \mathcal{D}_{\epsilon}^{\prime}$.

To state the next lemma, it is convenient to abbreviate

$$
g_{j}^{\circ}(z):=\frac{\lambda^{2}-\lambda_{+}^{2}}{\lambda^{2}} \frac{1}{\mu_{j}-z}, \quad G_{j j}^{\Delta}(z):=G_{j j}(z)-g_{j}^{\circ}(z), \quad\left(z \in \mathbb{C}^{+}\right) .
$$

We have the following estimate on $G_{j j}^{\Delta}(z)$.

Lemma 7.3. There are constants $C$ and $c$, such that, for all $z \in \mathcal{D}_{\epsilon}^{\prime}$, and all $j \leq n_{0}-1$,

$$
\left|G_{j j}^{\Delta}(z)\right| \leq C\left(\varphi_{N}\right)^{2 \xi}\left|g_{j}^{\circ}(z)\right|\left(\frac{1}{N^{2 /(\mathrm{b}+1)} \eta}+\frac{N^{3 \epsilon}}{N^{1 / 2} \eta}+\frac{(\kappa+\eta)^{\min \{\mathrm{b}, 2\}}}{\eta}\right),
$$

with $(\xi, \nu)$-high probability on $\Omega_{V}$.

Proof. For $z \in \mathcal{D}_{\epsilon}^{\prime}$, we have $\left(G_{j j}(z)\right)^{-1}=\lambda v_{j}-z-\widehat{m}_{f c}(z)+\mathcal{O}\left(N^{-1 / 2+2 \epsilon}\right)$, with high probability on $\Omega_{V}$; see Lemma 5.1. We thus obtain from (7.2) that

$$
G_{j j}(z)=g_{j}^{\circ}(z)+g_{j}^{\circ}(z) G_{j j}(z) \mathcal{O}\left(\left(\varphi_{N}\right)^{2 \xi} \frac{1}{N^{2 /(\mathrm{b}+1)}}+\frac{N^{3 \epsilon}}{N^{1 / 2}}+\left(\varphi_{N}\right)^{\xi}(\kappa+\eta)^{\min \{\mathrm{b}, 2\}}\right)
$$

with high probability on $\Omega_{V}$. Applying the trivial bound $\left|G_{j j}(z)\right| \leq \eta^{-1}$, Inequality (7.4) follows.

7.2. Proof of Theorem 2.11; Inequality (2.21). For $j \in \llbracket 1, N \rrbracket$, we define a weighted empirical eigenvalue counting measure, $\rho_{j}$, by

$$
\rho_{j}:=\sum_{k=1}^{N}\left|u_{k}(j)\right|^{2} \delta_{\mu_{k}}
$$

where $\left(\mu_{k}\right)$ are the eigenvalues of $H$ and $\left(u_{k}(j)\right)$ the components of the associated eigenvectors. For $k \in \llbracket 1, n_{0}-1 \rrbracket$, let $f_{k}(x) \geq 0$ be a smooth test function satisfying

$$
f_{k}(x)= \begin{cases}1, & \text { if }\left|x-\mu_{k}\right| \leq N^{-1 /(\mathrm{b}+1)-\epsilon^{\prime}} \\ 0, & \text { if }\left|x-\mu_{k}\right| \geq 2 N^{-1 /(\mathrm{b}+1)-\epsilon^{\prime}}, \quad\left|f_{k}^{\prime}(x)\right| \leq C N^{1 /(\mathrm{b}+1)} N^{\epsilon^{\prime}}, \quad\left|f_{k}^{\prime \prime}(x)\right| \leq C N^{2 /(\mathrm{b}+1)} N^{2 \epsilon^{\prime}}\end{cases}
$$

for some $\epsilon^{\prime}>\epsilon$. By Proposition 4.8, we know that the eigenvalues $\left(\mu_{k}\right)$ satisfy

$$
\min _{1 \leq k \leq n_{0}-1}\left(\mu_{k+1}-\mu_{k}\right) \geq C N^{-1 /(\mathrm{b}+1)-\epsilon},
$$

for some constant $C$, with high probability on $\Omega_{V}$. Since we chose $\epsilon^{\prime}>\epsilon$, we conclude that the following formula holds with high probability on $\Omega_{V}$, for all $k \leq n_{0}-1$,

$$
\left|u_{k}(j)\right|^{2}=\int f_{k}(w) \mathrm{d} \rho_{j}(w) .
$$


Using the Helffer-Sjöstrand formula, we may represent $f_{k}(w)$ as

$$
f_{k}(w)=\frac{1}{2 \pi} \int_{\mathbb{R}^{2}} \mathrm{~d} x \mathrm{~d} y \frac{\widetilde{f}_{k}(x+\mathrm{i} y)}{w-x-\mathrm{i} y}
$$

where

$$
\widetilde{f}_{k}(x+\mathrm{i} y):=\mathrm{i} y f_{k}^{\prime \prime}(x) \chi(y)+\mathrm{i}\left(f_{k}(x)+\mathrm{i} y f_{k}^{\prime}(x)\right) \chi^{\prime}(y), \quad(x, y \in \mathbb{R}),
$$

with $\chi \geq 0$ a smooth test function satisfying

$$
\chi(y)=\left\{\begin{array}{ll}
1, & \text { if } y \in[-\mathcal{E}, \mathcal{E}] \\
0, & \text { if } y \in[-2 \mathcal{E}, 2 \mathcal{E}]^{c}
\end{array}, \quad\left|\chi^{\prime}(y)\right| \leq \frac{C}{\mathcal{E}}\right.
$$

where we have set $\mathcal{E}:=N^{-1 /(\mathrm{b}+1)}$.

Combining (7.9) with (7.10), we obtain the following representation for $\left|u_{k}(j)\right|$, with $k \in \llbracket 1, n_{0}-1 \rrbracket, j \in \llbracket 1, N \rrbracket$,

$$
\left|u_{k}(j)\right|^{2}=\frac{1}{2 \pi} \int_{\mathbb{R}^{2}} \mathrm{~d} x \mathrm{~d} y \widetilde{f}_{k}(x+\mathrm{i} y) g_{j}^{\circ}(x+\mathrm{i} y)+\frac{1}{2 \pi} \int_{\mathbb{R}^{2}} \mathrm{~d} x \mathrm{~d} y \widetilde{f}_{k}(x+\mathrm{i} y) G_{j j}^{\Delta}(x+\mathrm{i} y),
$$

which holds with high probability on $\Omega_{V}$. The first term on the right side of (7.13) can be computed explicitly as

$$
\frac{1}{2 \pi} \int_{\mathbb{R}^{2}} \mathrm{~d} x \mathrm{~d} y \tilde{f}_{k}(x+\mathrm{i} y) g_{j}^{\circ}(x+\mathrm{i} y)=\frac{\lambda^{2}-\lambda_{+}^{2}}{2 \pi \lambda^{2}} \int_{\mathbb{R}^{2}} \frac{\tilde{f}_{k}(x+\mathrm{i} y)}{\mu_{j}-(x+\mathrm{i} y)}=\frac{\lambda^{2}-\lambda_{+}^{2}}{\lambda^{2}} f_{k}\left(\mu_{j}\right) .
$$

Recalling (7.7), we conclude by Proposition 4.8 that, for $j, k \leq n_{0}-1$, with high probability on $\Omega_{V}$,

$$
\frac{1}{2 \pi} \int_{\mathbb{R}^{2}} \mathrm{~d} x \mathrm{~d} y{\widetilde{f_{k}}}_{k}(x+\mathrm{i} y) g_{j}^{\circ}(x+\mathrm{i} y)=\frac{\lambda^{2}-\lambda_{+}^{2}}{\lambda^{2}} \delta_{j k}
$$

To complete the proof of (2.21), it hence suffices to show that the second term on the right side of (17.13) is negligible compared to (7.15), provided $\epsilon^{\prime}>\epsilon$. For concreteness we set $\epsilon^{\prime}=2 \epsilon$ in the following.

Lemma 7.4. Let $\widetilde{f}_{k}$ be defined as in (7.11) and set $\epsilon^{\prime}=2 \epsilon$. Then there are constants $C$ and $c$ such that, for $j, k \leq n_{0}-1$,

$$
\left|\int_{\mathbb{R}^{2}} \mathrm{~d} x \mathrm{~d} y \widetilde{f}_{k}(x+\mathrm{i} y) G_{j j}^{\Delta}(x+\mathrm{i} y)\right| \leq C\left(\varphi_{N}\right)^{c \xi}\left(N^{-1 /(\mathrm{b}+1)+3 \epsilon}+N^{-\mathfrak{b}+5 \epsilon}\right),
$$

with $(\xi, \nu)$-high probability on $\Omega_{V}$.

Proof. We set $\eta:=\frac{1}{\sqrt{N}}$. Using $G_{j j}^{\Delta}(\bar{z})=\overline{G_{j j}^{\Delta}(z)},\left(z \in \mathbb{C}^{+}\right)$, we obtain,

$$
\begin{aligned}
\left|\int_{\mathbb{R}^{2}} \mathrm{~d} x \mathrm{~d} y \widetilde{f}_{k}(x+\mathrm{i} y) G_{j j}^{\Delta}(x+\mathrm{i} y)\right| \leq & C \int \mathrm{d} x \int_{0}^{\infty} \mathrm{d} y\left|f_{k}(x)\right|\left|\chi^{\prime}(y)\right|\left|G_{j j}^{\Delta}(x+\mathrm{i} y)\right| \\
& +C \int \mathrm{d} x \int_{0}^{\infty} \mathrm{d} y y\left|f_{k}^{\prime}(x)\right|\left|\chi^{\prime}(y)\right|\left|G_{j j}^{\Delta}(x+\mathrm{i} y)\right| \\
& +C\left|\int \mathrm{d} x \int_{0}^{\eta} \mathrm{d} y f_{k}^{\prime \prime}(x) \chi(y) y \operatorname{Im} G_{j j}^{\Delta}(x+\mathrm{i} y)\right| \\
& +C\left|\int \mathrm{d} x \int_{\eta}^{\infty} \mathrm{d} y f_{k}^{\prime \prime}(x) \chi(y) y \operatorname{Im} G_{j j}^{\Delta}(x+\mathrm{i} y)\right| .
\end{aligned}
$$

The first term on the right side of (7.17) can be bounded by using (17.4): Bounding in (7.4) $\kappa \equiv \kappa_{x}$ by $\kappa \leq$ $N^{-1 /(\mathrm{b}+1)+\epsilon / 2}$ and $g_{j}^{\circ}$ by $\left|g_{j}^{\circ}(x+\mathrm{i} y)\right| \leq C y^{-1}$, we obtain

$$
\begin{gathered}
\int \mathrm{d} x \int_{0}^{\infty} \mathrm{d} y\left|f_{k}(x)\right|\left|\chi^{\prime}(y)\right|\left|G_{j j}^{\Delta}(x+\mathrm{i} y)\right| \leq \frac{C}{\mathcal{E}} \int \mathrm{d} x\left|f_{k}(x)\right| \int_{\mathcal{E}}^{2 \mathcal{E}} \mathrm{d} y\left|G_{j j}^{\Delta}(x+\mathrm{i} y)\right| \\
\leq C\left(\varphi_{N}\right)^{2 \xi} \frac{N^{-1 /(\mathrm{b}+1)-\epsilon^{\prime}}}{\mathcal{E}^{2}}\left(N^{-2 /(\mathrm{b}+1)}+N^{-1 / 2+3 \epsilon}+\left(N^{-1 /(\mathrm{b}+1)+\epsilon / 2}\right)^{\min \{\mathrm{b}, 2\}}\right) \\
\leq C\left(\varphi_{N}\right)^{2 \xi}\left(N^{-1 /(\mathrm{b}+1)-\epsilon^{\prime}+\epsilon}+N^{-\mathfrak{b}-\epsilon^{\prime}+3 \epsilon}\right)
\end{gathered}
$$


with high probability on $\Omega_{V}$.

Similarly, we bound the second term on the right side of (7.17):

$$
\begin{aligned}
\int \mathrm{d} x \int_{0}^{\infty} \mathrm{d} y y & \left|f_{k}^{\prime}(x)\right|\left|\chi^{\prime}(y)\right|\left|G_{j j}^{\Delta}(x+\mathrm{i} y)\right| \\
& \leq \frac{C}{\mathcal{E}} \int \mathrm{d} x\left|f_{k}^{\prime}(x)\right| \int_{\mathcal{E}}^{2 \mathcal{E}} \mathrm{d} y y\left|G_{j j}^{\Delta}(x+\mathrm{i} y)\right| \\
& \leq \frac{C\left(\varphi_{N}\right)^{2 \xi}}{\mathcal{E}}|\log \mathcal{E}|\left(N^{-2 /(\mathrm{b}+1)}+N^{-1 / 2+3 \epsilon}+\left(N^{-1 /(\mathrm{b}+1)+\epsilon / 2}\right)^{\min \{\mathrm{b}, 2\}}\right) \\
& \leq C\left(\varphi_{N}\right)^{c \xi}\left(N^{-1 /(\mathrm{b}+1)+\epsilon}+N^{-\mathfrak{b}+3 \epsilon}\right) .
\end{aligned}
$$

To bound the third term on the right side of (7.17), we use that $\left|y \operatorname{Im} G_{j j}^{\Delta}(x+\mathrm{i} y)\right| \leq C$, for all $y>0$, and we obtain

$$
\left|\int \mathrm{d} x \int_{0}^{\eta} \mathrm{d} y f_{k}^{\prime \prime}(x) \chi(y) y \operatorname{Im} G_{j j}^{\Delta}(x+\mathrm{i} y)\right| \leq C \eta \int \mathrm{d} x\left|f_{k}^{\prime \prime}(x)\right| \leq C N^{-\mathfrak{b}+\epsilon^{\prime}} .
$$

To bound the fourth term in (7.17), we integrate by parts, first in $x$ then in $y$ to find the bound

$$
\begin{aligned}
C\left|\int \mathrm{d} x f_{k}^{\prime}(x) \eta \operatorname{Re} G_{j j}^{\Delta}(x+\mathrm{i} \eta)\right|+C & \left|\int \mathrm{d} x \int_{\eta}^{\infty} \mathrm{d} y f_{k}^{\prime}(x) \chi^{\prime}(y) y \operatorname{Re} G_{j j}^{\Delta}(x+\mathrm{i} y)\right| \\
& +C\left|\int \mathrm{d} x \int_{\eta}^{\infty} \mathrm{d} y f_{k}^{\prime}(x) \chi(y) \operatorname{Re} G_{j j}^{\Delta}(x+\mathrm{i} y)\right| .
\end{aligned}
$$

The first term in (7.22) can be bounded using (7.4): Since $f_{k}^{\prime}(x)=0$, if $\left|x-\mu_{k}\right| \leq N^{-1 /(\mathrm{b}+1)-\epsilon^{\prime}}$, we can bound $\left|f_{k}^{\prime}(x) g_{j}^{\circ}(x+\mathrm{i} y)\right| \leq C N^{2 /(\mathrm{b}+1)+2 \epsilon^{\prime}}$, and we obtain

$$
\begin{aligned}
& \left|\int \mathrm{d} x f_{k}^{\prime}(x) \eta \operatorname{Re} G_{j j}^{\Delta}(x+\mathrm{i} \eta)\right| \\
& \quad \leq C\left(\varphi_{N}\right)^{2 \xi} N^{1 /(\mathrm{b}+1)+\epsilon^{\prime}} \eta\left(\frac{1}{N^{2 /(\mathrm{b}+1)} \eta}+\frac{N^{3 \epsilon}}{N^{1 / 2} \eta}+\frac{\left.\left(N^{-1 /(\mathrm{b}+1)+\epsilon / 2}\right)^{\min \{\mathrm{b}, 2\}}\right)}{\eta}\right) \\
& \quad \leq C\left(\varphi_{N}\right)^{2 \xi}\left(N^{-1 /(\mathrm{b}+1)+\epsilon^{\prime}+\epsilon}+N^{-\mathfrak{b}+\epsilon^{\prime}+3 \epsilon}\right),
\end{aligned}
$$

with high probability on $\Omega_{V}$.

Similarly for the second term in (7.22), using (7.4) we obtain

$$
\left|\int \mathrm{d} x \int_{\eta}^{\infty} \mathrm{d} y f_{k}^{\prime}(x) \chi^{\prime}(y) y \operatorname{Re} G_{j j}^{\Delta}(x+\mathrm{i} y)\right| \leq C\left(\varphi_{N}\right)^{2 \xi}\left(N^{-1 /(\mathrm{b}+1)+\epsilon^{\prime}+\epsilon}+N^{-\mathfrak{b}+\epsilon^{\prime}+3 \epsilon}\right) .
$$

The third term in (7.22) can be bounded using (7.4) as

$$
\begin{aligned}
\mid \int \mathrm{d} x & \int_{\eta}^{\infty} \mathrm{d} y f_{k}^{\prime}(x) \chi(y) \operatorname{Re} G_{j j}^{\Delta}(x+\mathrm{i} y) \mid \\
& \leq C\left(\varphi_{N}\right)^{2 \xi} N^{1 /(\mathrm{b}+1)+\epsilon^{\prime}} \int_{\eta}^{2 \mathcal{E}} \mathrm{d} y\left(\frac{1}{N^{2 /(\mathrm{b}+1)} y}+\frac{N^{3 \epsilon}}{N^{1 / 2} y}+\frac{\left.\left(N^{-1 /(\mathrm{b}+1)+\epsilon / 2}\right)^{\min \{\mathrm{b}, 2\}}\right)}{y}\right) \\
& \leq C|\log \eta|\left(\varphi_{N}\right)^{2 \xi}\left(N^{-1 /(\mathrm{b}+1)+\epsilon^{\prime}+\epsilon}+N^{-\mathfrak{b}+\epsilon^{\prime}+3 \epsilon}\right) .
\end{aligned}
$$

Thus combining (7.18), (7.21), (7.23), (7.24) and (7.25), we obtain, upon choosing $\epsilon^{\prime}=2 \epsilon$,

$$
\left|\int \mathrm{d} x \mathrm{~d} y \widetilde{f}_{k}(x+\mathrm{i} y) G_{i i}^{\Delta}(x+\mathrm{i} y)\right| \leq C\left(\varphi_{N}\right)^{c \xi}\left(N^{-1 /(\mathrm{b}+1)+3 \epsilon}+N^{-\mathfrak{b}+5 \epsilon}\right),
$$

with high probability on $\Omega_{V}$.

Proof of Theorem 2.8; Equation (2.21). Combining (7.13), (7.15) and (17.16), we obtain, for $j, k \leq n_{0}-1$,

$$
\left|u_{k}(j)\right|^{2}=\frac{\lambda^{2}-\lambda_{+}^{2}}{\lambda^{2}} \delta_{j k}+\mathcal{O}\left(\left(\varphi_{N}\right)^{c \xi} N^{-1 /(\mathrm{b}+1)+3 \epsilon}+\left(\varphi_{N}\right)^{c \xi} N^{-\mathfrak{b}+5 \epsilon}\right),
$$

with high probability on $\Omega_{V}$. Choosing $k=j$, Equation (2.21) follows. 
7.3. Proof of Theorem 2.11; Inequality (2.22). In this subsection we prove the second part of Theorem 2.11, To prove (2.22), we follow the traditional path of 20, 21, 22. Presumably, the same result can be obtained by a more detailed analysis of $G_{j j}^{\Delta}$ than the one carried out in the previous subsection.

Recall that we have set $\eta_{0}:=N^{-1 / 2-\epsilon}$.

Lemma 7.5. There is a constant $C$, such that for $k \in \llbracket 1, n_{0}-1 \rrbracket, j \in \llbracket 1, N \rrbracket,(k \neq j)$,

$$
\operatorname{Im} G_{j j}\left(\mu_{k}+\mathrm{i} \eta_{0}\right) \leq \frac{C}{\left(\lambda v_{j}-\lambda v_{k}\right)^{2}} N^{-1 / 2+3 \epsilon}
$$

with $(\xi, \nu)$-high probability, on $\Omega_{V}$.

Proof. For $z \in \mathcal{D}_{\epsilon}^{\prime}$, we have

$$
\left(G_{j j}(z)\right)^{-1}=\lambda v_{j}-z-\widehat{m}_{f c}(z)+\mathcal{O}\left(N^{-1 / 2+2 \epsilon}\right),
$$

with high probability on $\Omega_{V}$; see Lemma 5.1. Next, recall from Proposition 4.6, we have with high probability on $\Omega_{V}$ that

$$
\mu_{k}+\operatorname{Re} \widehat{m}_{f_{c}}\left(\mu_{k}+\mathrm{i} \eta_{0}\right)=\lambda v_{k}+\mathcal{O}\left(N^{-1 / 2+3 \epsilon}\right) .
$$

Also recall the high probability estimate $\operatorname{Im} m(z) \leq \frac{N^{2 \epsilon}}{\sqrt{N}}$, for $z \in \mathcal{D}_{\epsilon}^{\prime}$, on $\Omega_{V}$; see Lemma 5.4. Thus, choosing $z=\mu_{k}+\mathrm{i} \eta_{0}$, with $k \in \llbracket 1, n_{0}-1 \rrbracket$ and $k \neq j$, in (7.29) we obtain

$$
\begin{aligned}
\left(G_{j j}\left(\mu_{k}+\mathrm{i} \eta_{0}\right)\right)^{-1} & =\lambda v_{j}-\mu_{k}-\mathrm{i} \eta_{0}-\widehat{m}_{f c}\left(\mu_{k}+\mathrm{i} \eta_{o}\right)+\mathcal{O}\left(N^{-1 / 2+2 \epsilon}\right) \\
& =\lambda v_{j}-\lambda v_{k}+\mathcal{O}\left(N^{-1 / 2+3 \epsilon}\right),
\end{aligned}
$$

with high probability on $\Omega_{V}$. Since $\left|v_{j}-v_{k}\right| \geq C N^{-1 /(\mathrm{b}+1)-\epsilon}, j \neq k$, on $\Omega_{V}$, we obtain

$$
\operatorname{Im} G_{j j}\left(\mu_{k}+\mathrm{i} \eta_{0}\right) \leq \frac{C}{\left(\lambda v_{j}-\lambda v_{k}\right)^{2}} N^{-1 / 2+3 \epsilon},
$$

with high probability on $\Omega_{V}$, for some constant $C$.

Proof of Theorem 2.11: Inequality (2.22). From the spectral decomposition of the resolvent of $H$, we obtain

$$
\operatorname{Im} G_{j j}\left(\mu_{k}+\mathrm{i} \eta_{0}\right)=\sum_{\alpha=1}^{N} \frac{\left|u_{\alpha}(j)\right|^{2} \eta_{0}}{\left(\mu_{\alpha}-\mu_{k}\right)^{2}+\eta_{0}^{2}} \geq \frac{1}{\eta_{0}}\left|u_{k}(j)\right|^{2},
$$

thus, using the bound (7.28), we conclude that

$$
\left|u_{k}(j)\right|^{2} \leq C \frac{\eta_{0}}{\left(\lambda v_{k}-\lambda v_{j}\right)^{2}} N^{-1 / 2+3 \epsilon} \leq C \frac{1}{\left(\lambda v_{k}-\lambda v_{j}\right)^{2}} N^{-1+2 \epsilon},
$$

with high probability on $\Omega_{V}$, for $k \in \llbracket 1, n_{0}-1 \rrbracket, j \in \llbracket 1, N \rrbracket,(j \neq k)$. This completes the proof of Theorem 2.11

\section{Appendix A}

In this appendix, we estimate the probabilities for the events 1.-3. in the definition of $\Omega_{V}$; see Definition 4.1 Recall the definition of the constants $\epsilon$ in (4.2) and $\kappa_{0}$ in (4.5). In the following, we denote, unlike in the rest of the paper, by $\left(v_{i}\right)_{i=1}^{N}$ (unordered) sample points distributed according to the measure $\mu$, (with b $>1$ ). We denote by $\left(v_{(i)}\right.$ ) the order statistics of $\left(v_{i}\right)$ with the convention $v_{(1)} \geq v_{(2)} \geq \ldots \geq v_{(N)}$.

Lemma A.1. Let $\left(v_{(i)}\right)$ be the order statistics of sample points $\left(v_{i}\right)$ under the probability distribution $\mu$ with $\mathrm{b}>1$. Let $n_{0}>10$ be a fixed positive integer independent of $N$. Then, for any $k \in \llbracket 1, n_{0}-1 \rrbracket$ and for any sufficiently small $\epsilon>0$, we have

$$
\mathbb{P}\left(N^{-\epsilon} \kappa_{0}<\left|v_{(k)}-v_{(j)}\right|<(\log N) \kappa_{0}, \forall j \neq k\right) \geq 1-C(\log N)^{1+2 \mathrm{~b}} N^{-\epsilon} .
$$

In addition, for $k=1$, we have

$$
\mathbb{P}\left(N^{-\epsilon} \kappa_{0}<\left|1-v_{(1)}\right|<(\log N) \kappa_{0}\right) \geq 1-C N^{-\epsilon(\mathrm{b}+1)} .
$$

Proof. Consider the following claims: 
(1) There exists a constant $C>0$ such that

$$
\mathbb{P}\left(\left|1-v_{(1)}\right|>N^{-\epsilon} \kappa_{0}\right) \geq 1-C N^{-\epsilon(\mathrm{b}+1)} .
$$

(2) There exists a constant $c>0$ such that

$$
\mathbb{P}\left(\left|1-v_{\left(n_{0}\right)}\right|>(\log N) \kappa_{0}\right) \leq e^{-c(\log N)^{\mathrm{b}+1}} .
$$

(3) There exists a constant $C>0$ such that, for any $k \in \llbracket 1, n_{0}-1 \rrbracket$,

$$
\mathbb{P}\left(\left|v_{(k)}-v_{(k+1)}\right| \leq N^{-\epsilon} \kappa_{0}\right) \leq C(\log N)^{1+2 \mathrm{~b}} N^{-\epsilon}
$$

Assuming the claims (1)-(3), it is easy to see that the desired lemma holds.

For a random variables $v$ with law $\mu$ as in (2.10), we have for any $x \geq 0$,

$$
C^{-1} x^{\mathrm{b}+1} \leq \mathbb{P}(1-v \leq x) \leq C x^{\mathrm{b}+1},
$$

for some constant $C>1$. Thus, we obtain for the first order statistics of $\left(v_{i}\right)$ that

$$
\mathbb{P}\left(\left|1-v_{(1)}\right|>N^{-\epsilon} \kappa_{0}\right)=\left(1-\mathbb{P}\left(1-v \leq N^{-\epsilon} \kappa_{0}\right)\right)^{N} \geq\left(1-C N^{-\epsilon(\mathrm{b}+1)} N^{-1}\right)^{N} \geq 1-C N^{-\epsilon(\mathrm{b}+1)}
$$

proving claim (1).

Similarly, we have

$$
\begin{aligned}
& \mathbb{P}\left(\left|1-v_{\left(n_{0}\right)}\right|>(\log N) \kappa_{0}\right) \leq\left(\begin{array}{c}
N \\
n_{0}
\end{array}\right)\left(1-\mathbb{P}\left(1-v \leq(\log N) \kappa_{0}\right)\right)^{N-n_{0}} \\
& \leq N^{n_{0}}\left(1-C^{-1}(\log N)^{\mathrm{b}+1} N^{-1}\right)^{N-n_{0}} \leq C N^{n_{0}} e^{-c(\log N)^{\mathrm{b}+1}} \leq e^{-c^{\prime}(\log N)^{\mathrm{b}+1}},
\end{aligned}
$$

for some constant $c, c^{\prime}>0$, proving claim (2).

To prove claim (3), we assume that $N^{-\epsilon} \kappa_{0}<\left|1-v_{(1)}\right| \leq\left|1-v_{\left(n_{0}\right)}\right| \leq(\log N) \kappa_{0}$, which holds with probability higher than $1-C N^{-\epsilon(\mathrm{b}+1)}$. Let

$$
I_{j}:=\left[1-(j+1) N^{-\epsilon} \kappa_{0}, 1-(j-1) N^{-\epsilon} \kappa_{0}\right], \quad\left(j \in \llbracket 1,(\log N) N^{\epsilon} \rrbracket\right) .
$$

Then, it can easily be seen that if $\left|v_{(k)}-v_{(k+1)}\right|>N^{-\epsilon} \kappa_{0}$, then $v_{(k)}, v_{(k+1)} \in I_{j}$ for some $j \in \llbracket 1,(\log N) N^{\epsilon} \rrbracket$. Letting $p_{j}:=\mathbb{P}\left(v \in I_{j}\right)$, we have that

$$
\mathbb{P}\left(\left|\left\{i \in \llbracket 1, N \rrbracket: v_{i} \in I_{j}\right\}\right|=0\right)=\left(1-p_{j}\right)^{N}, \quad \mathbb{P}\left(\left|\left\{i \in \llbracket 1, N \rrbracket: v_{i} \in I_{j}\right\}\right|=1\right)=N p_{j}\left(1-p_{j}\right)^{N-1},
$$

hence,

$$
\mathbb{P}\left(v_{(k)}, v_{(k+1)} \in I_{j}\right) \leq \mathbb{P}\left(\left|\left\{i \in \llbracket 1, N \rrbracket: v_{i} \in I_{j}\right\}\right|=2\right) \leq 1-\left(1-p_{j}\right)^{N}-N p_{j}\left(1-p_{j}\right)^{N-1} \leq N^{2} p_{j}^{2} .
$$

Since

$$
p_{j} \leq C N^{-\epsilon} \kappa_{0}\left((\log N) \kappa_{0}\right)^{\mathrm{b}}=C(\log N)^{\mathrm{b}} N^{-1-\epsilon},
$$

we have

$$
\mathbb{P}\left(\left|v_{(k)}-v_{(k+1)}\right| \leq N^{-\epsilon} \kappa_{0}\right) \leq \sum_{j \in \llbracket 1,(\log N) N \epsilon \rrbracket} N^{2} p_{j}^{2} \leq C(\log N)^{1+2 \mathrm{~b}} N^{-\epsilon}
$$

This proves the third part of the claim and completes the proof of the lemma.

Next, we estimate the probability of condition (2) in Definition 4.1 to hold.

Lemma A.2. Assume the conditions in Lemma A.1. Recall the definition of $\mathcal{D}_{\epsilon}$ in (4.3). Then, for any fixed $\ell>0$, there exists a constant $C_{\ell}$ (independent of $N$ ) such that

$$
\mathbb{P}\left(\bigcup_{z \in \mathcal{D}_{\epsilon}}\left\{\left|\frac{1}{N} \sum_{i=1}^{N} \frac{1}{\lambda v_{i}-z-m_{f c}(z)}-\int \frac{\mathrm{d} \mu(v)}{\lambda v-z-m_{f c}(z)}\right|>\frac{N^{3 \epsilon / 2}}{\sqrt{N}}\right\}\right) \leq C_{\ell} N^{-\ell}
$$


Proof. Fix $z \in \mathcal{D}_{\epsilon}$. For $i \in \llbracket 1, N \rrbracket$, let $X_{i} \equiv X_{i}(z)$ be the random variable

$$
X_{i}:=\frac{1}{\lambda v_{i}-z-m_{f c}(z)}-m_{f c}(z)=\frac{1}{\lambda v_{i}-z-m_{f c}(z)}-\int \frac{\mathrm{d} \mu(v)}{\lambda v-z-m_{f c}(z)} .
$$

By definition, $\mathbb{E} X_{i}=0$. Moreover, we have

$$
\mathbb{E}\left|X_{i}\right|^{2} \leq \int \frac{\mathrm{d} \mu(v)}{\left|\lambda v-z-m_{f c}(z)\right|^{2}}=\frac{\operatorname{Im} m_{f c}((z)}{\eta+\operatorname{Im} m_{f c}(z)}<1, \quad\left(z \in \mathbb{C}^{+}\right),
$$

and, for any positive integer $p \geq 2$,

$$
\mathbb{E}\left|X_{i}\right|^{p} \leq \frac{1}{\eta^{p-2}} \mathbb{E}\left|X_{i}\right|^{2} \leq N^{(1 / 2+\epsilon)(p-2)}, \quad\left(z \in \mathcal{D}_{\epsilon}\right) .
$$

We now consider

$$
\mathbb{E}\left|\frac{1}{N} \sum_{i=1}^{N} X_{i}\right|^{2 p}=\frac{1}{N^{2 p}} \sum_{i_{1}, i_{2}, \ldots, i_{2 p}=1}^{N} \mathbb{E} \prod_{j=1}^{p} X_{i_{j}} \prod_{k=p+1}^{2 p} \bar{X}_{i_{k}} .
$$

For fixed $i_{1}, i_{2}, \ldots, i_{2 p}$, define

$$
d_{i}:=\sum_{j=1}^{2 p} \mathbb{1}\left(i_{j}=i\right)
$$

for $i \in \llbracket 1, N \rrbracket$.

We first estimate the summand in the right side of (A.9) when $d_{1} \geq d_{2} \geq \ldots \geq d_{r} \geq 1$ and $d_{r+1}=d_{r+2}=\ldots=$ $d_{N}=0$, for some $r \in \llbracket 1,2 p \rrbracket$. Since $\left(X_{i}\right)$ are independent and centered, we have

$$
\mathbb{E} \prod_{j=1}^{p} X_{i_{j}} \prod_{k=p+1}^{2 p} \bar{X}_{i_{k}}=0
$$

if $d_{i}=1$, for some $i$, which shows that we may assume $r \leq p$ and $d_{r} \geq 2$. When $d_{r} \geq 2$, we obtain that

$$
\left|\mathbb{E} \prod_{j=1}^{p} X_{i_{j}} \prod_{k=p+1}^{2 p} \bar{X}_{i_{k}}\right| \leq \mathbb{E} \prod_{k=1}^{r}\left|X_{k}\right|^{d_{k}} \leq \prod_{k=1}^{r} N^{(1 / 2+\epsilon)\left(d_{k}-2\right)} \leq N^{(1 / 2+\epsilon)(2 p-2 r)} .
$$

Next, using the estimate obtained above, we can bound

$$
\begin{aligned}
& \sum_{i_{1}, i_{2}, \ldots, i_{2 p}} \mathbb{E} \prod_{j=1}^{p} X_{i_{j}} \prod_{k=p+1}^{2 p} \bar{X}_{i_{k}} \\
& \quad \leq \sum_{r=1}^{p} r !\left(\begin{array}{c}
N \\
r
\end{array}\right) N^{(1 / 2+\epsilon)(2 p-2 r)} \leq p ! \sum_{r=1}^{p} N^{r} N^{(1 / 2+\epsilon)(2 p-2 r)} \leq(p+1) ! N^{p} N^{2 \epsilon p} .
\end{aligned}
$$

Hence, by Markov's inequality, we obtain that

$$
\mathbb{P}\left(\left|\frac{1}{N} \sum_{i=1}^{N} X_{i}\right| \geq \frac{N^{3 \epsilon / 2}}{2 \sqrt{N}}\right) \leq\left(\frac{1}{2} N^{3 \epsilon / 2}\right)^{-2 p} N^{p} \mathbb{E}\left|\frac{1}{N} \sum_{i=1}^{N} X_{i}\right|^{2 p} \leq 4^{p}(p+1) ! N^{-\epsilon p} .
$$

To obtain the uniform bound on $\mathcal{D}_{\epsilon}$, we choose a lattice $\mathcal{L}$ in $\mathcal{D}_{\epsilon}$ such that for any $z \in \mathcal{D}_{\epsilon}$ there exists $z^{\prime} \in \mathcal{L}$ satisfying $\left|z-z^{\prime}\right| \leq N^{-2}$. Since, for $z \in \mathcal{D}_{\epsilon}$ and $z^{\prime} \in \mathcal{L}$,

$$
\left|\frac{1}{\lambda v-z-m_{f c}(z)}-\frac{1}{\lambda v-z^{\prime}-m_{f c}\left(z^{\prime}\right)}\right| \leq C \frac{1}{\eta_{0}^{2}}\left|z-z^{\prime}\right| \leq C N^{-1+2 \epsilon},
$$

we find that

$$
\begin{aligned}
\mathbb{P}\left(\bigcup_{z \in \mathcal{D}_{\epsilon}}\left\{\left|\frac{1}{N} \sum_{i=1}^{N} \frac{1}{\lambda v_{i}-z-m_{f c}(z)}-m_{f c}(z)\right|>\frac{N^{3 \epsilon / 2}}{\sqrt{N}}\right\}\right) & \leq|\mathcal{L}| 4^{p}(p+1) ! N^{-\epsilon p} \\
& \leq C N^{4} 4^{p}(p+1) ! N^{-\epsilon p}
\end{aligned}
$$

Setting $p=(\ell+4) / \epsilon$, we obtain the desired lemma. 
To estimate the probability for the third condition in Definition 4.1, we need the following two auxiliary lemmas. Recall the definition of $R_{2}$ in (4.15).

Lemma A.3. If $0<C^{-1} \eta \leq \operatorname{Im} m_{f c}(z) \leq C \eta, z=E+\mathrm{i} \eta$, for some constant $C \geq 1$, then we have

$$
\frac{1}{1+C} \leq R_{2}(z) \leq \frac{C}{1+C}
$$

Proof. Since

$$
\operatorname{Im} m_{f c}(z)=\int \frac{\operatorname{Im} z+\operatorname{Im} m_{f c}(z)}{\left|\lambda v-z-m_{f c}(z)\right|^{2}} \mathrm{~d} \mu(v)
$$

we have that

$$
\frac{C^{-1}}{1+C^{-1}} \leq R_{2}(z)=\frac{\operatorname{Im} m_{f c}(z)}{\operatorname{Im} z+\operatorname{Im} m_{f c}(z)} \leq \frac{C}{1+C}
$$

The imaginary part of $m_{f c}(z)$ can be estimated using the following lemma.

Lemma A.4. Assume that $\mu_{f c}$ has support $\left[L_{-}, L_{+}\right]$and there exists a constant $C>1$ such that

$$
C^{-1} \kappa^{\mathrm{b}} \leq \mu_{f c}(z) \leq C \kappa^{\mathrm{b}}
$$

for any $0 \leq \kappa \leq L_{+}$. Then,

(1) for $z=L_{+}-\kappa+i \eta$ with $0 \leq \kappa \leq L_{+}$and $0<\eta \leq 3$, there exists a constant $C>1$ such that

$$
C^{-1}\left(\kappa^{\mathrm{b}}+\eta\right) \leq \operatorname{Im} m_{f c}(z) \leq C\left(\kappa^{\mathrm{b}}+\eta\right)
$$

(2) for $z=L_{+}+\kappa+i \eta$ with $0 \leq \kappa \leq 1$ and $0<\eta \leq 3$, there exists a constant $C>1$ such that

$$
C^{-1} \eta \leq \operatorname{Im} m_{f c}(z) \leq C \eta
$$

Remark A.5. Lemma A.4 shows that there exists a constant $C_{\mathrm{b}}>1$ such that

$$
C_{\mathrm{b}}^{-1} \eta \leq \operatorname{Im} m_{f c}(z) \leq C_{\mathrm{b}} \eta,
$$

for all $z \in \mathcal{D}_{\epsilon}$ satisfying $L_{+}-\operatorname{Re} z \leq N^{\epsilon} \kappa_{0}$.

Assuming Lemma A.4, we have the following estimate. Recall that $\mathcal{D}_{\epsilon}$ is defined in (4.3).

Lemma A.6. Assume the conditions in Lemma A.1. Then, there exist constants $\mathfrak{c}<1$ and $C>0$, independent of $N$, such that, for any $z=E+\mathrm{i} \eta \in \mathcal{D}_{\epsilon}$ satisfying

$$
\min _{i \in \llbracket 1, N \rrbracket}\left|\operatorname{Re}\left(z+m_{f c}(z)\right)-\lambda v_{(i)}\right|=\left|\operatorname{Re}\left(z+m_{f c}(z)\right)-\lambda v_{(k)}\right|,
$$

for some $k \in \llbracket 1, n_{0}-1 \rrbracket$, we have

$$
\mathbb{P}\left(\frac{1}{N} \sum_{i: i \neq k}^{N} \frac{1}{\left|\lambda v_{(i)}-z-m_{f c}(z)\right|^{2}}<\mathfrak{c}\right) \geq 1-C(\log N)^{1+2 \mathrm{~b}} N^{-\epsilon}
$$

Proof. Without loss of generality, we only prove the case $k=1$; the general case can easily be shown by using the same argument. In the following, we assume that $N^{-\epsilon} \kappa_{0}<\left|1-v_{(1)}\right|<(\log N) \kappa_{0}$, and $\left|v_{(1)}-v_{(2)}\right|>N^{-\epsilon} \kappa_{0}$.

Recall the definition of $R_{2}$ in (4.15). For $i \in \llbracket 1, N \rrbracket$, let $Y_{i} \equiv Y_{i}(z)$ be the random variable

$$
Y_{i}(z):=\frac{1}{\left|\lambda v_{i}-z-m_{f c}(z)\right|^{2}}, \quad\left(z \in \mathbb{C}^{+}\right),
$$

and observe that $\mathbb{E} Y_{i}=R_{2}<1,\left(z \in \mathbb{C}^{+}\right)$. Moreover, combining Lemma A.3 and Lemma A.4, we find that there exists a constant $c<1$, independent of $N$, such that that $R_{2}(z)<c$ uniformly for all $z \in \mathcal{D}_{\epsilon}$ satisfying (A.17). Since $\operatorname{Im}\left(z+m_{f c}(z)\right) \geq \eta$, we also have that $Y_{i}(z) \leq \eta^{-2}$.

We first consider the special choice $E=L_{+}$. Let $\tilde{Y}_{i}$ be the truncated random variable defined by

$$
\tilde{Y}_{i}:= \begin{cases}Y_{i}, & \text { if } Y_{i}<N^{2 \epsilon} \kappa_{0}^{-2} \\ N^{2 \epsilon} \kappa_{0}^{-2}, & \text { if } Y_{i} \geq N^{2 \epsilon} \kappa_{0}^{-2}\end{cases}
$$


Notice that, using the estimate (A.3), we have for $z=L_{+}+\mathrm{i} \eta \in \mathcal{D}_{\epsilon}$ that

$$
\mathbb{P}\left(Y_{i} \neq \tilde{Y}_{i}\right) \leq C N^{-1-(\mathrm{b}+1) \epsilon} .
$$

Let

$$
S_{N}:=\sum_{i=1}^{N} Y_{i}, \quad \widetilde{S}_{N}:=\sum_{i=1}^{N} \widetilde{Y}_{i}
$$

Then,

$$
\mathbb{P}\left(S_{N} \neq \widetilde{S}_{N}\right) \leq C N^{-(\mathrm{b}+1) \epsilon} .
$$

We now estimate the mean and variance of $\tilde{Y}_{i}$. From the trivial estimate $\mathbb{P}\left(Y_{i} \geq x\right) \leq \mathbb{P}\left(Y_{i} \neq \tilde{Y}_{i}\right)$ for $x \geq N^{2 \epsilon} \kappa_{0}^{-2}$, we find that

$$
\mathbb{E} Y_{i}-\mathbb{E} \tilde{Y}_{i} \leq \int_{N^{2 \epsilon} \kappa_{0}^{-2}}^{\eta^{-2}} \mathbb{P}\left(Y_{i} \neq \widetilde{Y}_{i}\right) \mathrm{d} x \leq C^{\prime} N^{-(\mathrm{b}-1) \epsilon},
$$

for some $C^{\prime}>0$. Hence, we get

$$
\mathbb{E} \tilde{Y}_{i}^{2} \leq N^{2 \epsilon} \kappa_{0}^{-2} \mathbb{E} \tilde{Y}_{i} \leq N^{2 \epsilon} \kappa_{0}^{-2} \mathbb{E} Y_{i} \leq N^{2 \epsilon} \kappa_{0}^{-2}
$$

We thus obtain that

$$
\begin{gathered}
\mathbb{P}\left(\left|\frac{S_{N}}{N}-\mathbb{E} Y_{i}\right| \geq C^{\prime} N^{-(\mathrm{b}-1) \epsilon}+N^{-\epsilon}\right) \leq \mathbb{P}\left(\left|\frac{\widetilde{S}_{N}}{N}-\mathbb{E} \widetilde{Y}_{i}\right| \geq N^{-\epsilon}\right)+\mathbb{P}\left(S_{N} \neq \widetilde{S}_{N}\right) \\
\leq \frac{N^{2 \epsilon} \mathbb{E} \widetilde{Y}_{i}^{2}}{N}+C N^{-(\mathrm{b}+1) \epsilon} \leq C N^{-\frac{\mathrm{b}-1}{\mathrm{~b}+1}+4 \epsilon}=C N^{-2 \mathfrak{b}+4 \epsilon}
\end{gathered}
$$

hence, for a constant $c$ satisfying $R_{2}+C^{\prime} N^{-(\mathrm{b}-1) \epsilon}+N^{-\epsilon}<c<1$,

$$
\mathbb{P}\left(\frac{1}{N} \sum_{i=1}^{N} \frac{1}{\left|\lambda v_{i}-z-m_{f c}(z)\right|^{2}}<c\right) \geq 1-\mathbb{P}\left(\left|\frac{S_{N}}{N}-\mathbb{E} Y_{i}\right| \geq C^{\prime} N^{-(\mathrm{b}-1) \epsilon}+N^{-\epsilon}\right) \geq 1-C N^{-2 \mathfrak{b}+4 \epsilon} .
$$

This proves the desired lemma for $E=L_{+}$.

Before we extend the result to general $z \in \mathcal{D}_{\epsilon}$, we estimate the probabilities for some typical events we want to assume. Consider the set

$$
\Sigma_{\epsilon}:=\left\{v_{i}:\left|1-v_{i}\right| \leq N^{3 \epsilon} \kappa_{0}\right\},
$$

and the event

$$
\Omega_{\epsilon}:=\left\{\left|\Sigma_{\epsilon}\right|<N^{3 \epsilon(\mathrm{b}+2)}\right\} .
$$

Since we have from the estimate (A.3) that

$$
\mathbb{P}\left(\left|1-v_{i}\right| \geq N^{3 \epsilon} \kappa_{0}\right) \leq C N^{-1+3(\mathrm{~b}+1) \epsilon},
$$

we find, using a Chernoff bound, that

$$
\mathbb{P}\left(\Omega_{\epsilon}^{c}\right) \leq \exp \left(-C \epsilon(\log N) N^{3 \epsilon} N^{3(\mathrm{~b}+1) \epsilon}\right),
$$

for some constant $C$. Notice that we have, for $v_{i} \notin \Sigma_{\epsilon}$,

$$
L_{+}+\operatorname{Re} m_{f c}\left(L_{+}+\mathrm{i} \eta\right)-\lambda v_{i}>N^{3 \epsilon} \kappa_{0} \gg \eta+\operatorname{Im} m_{f c}\left(L_{+}+\mathrm{i} \eta\right),
$$

where we used Lemma 4.2, i.e., $\left|L_{+}+\mathrm{i} \eta+m_{f c}\left(L_{+}+\mathrm{i} \eta\right)-\lambda\right|=\mathcal{O}(\eta)$, and that $\lambda>1$. We now assume that $\Omega_{\epsilon}$ holds and that

$$
\frac{1}{N} \sum_{i=1}^{N} \frac{1}{\left|\lambda v_{i}-\left(L_{+}+\mathrm{i} \eta\right)-m_{f c}\left(L_{+}+\mathrm{i} \eta\right)\right|^{2}}<c<1
$$

Further, we recall that the condition (A.17) implies

$$
\operatorname{Re}\left(z+m_{f c}(z)\right) \geq \lambda v_{\left(n_{0}\right)}
$$

which yields, together with Lemma 4.2 and Lemma A.1, that $E \geq L_{+}-N^{\epsilon} \kappa_{0}$ with probability higher than $1-C(\log N)^{1+2 \mathrm{~b}} N^{-\epsilon}$. We therefore assume in the following that $E \geq L_{+}-N^{\epsilon} \kappa_{0}$.

Consider the following two choices for such energies $E$ : 
(1) When $L_{+}-N^{\epsilon} \kappa_{0} \leq E \leq L_{+}+N^{2 \epsilon} \kappa_{0}$, we have that

$$
\left|\lambda v_{i}-z-m_{f c}(z)\right|=\left|\lambda v_{i}-\left(L_{+}+\mathrm{i} \eta\right)-m_{f c}\left(L_{+}+\mathrm{i} \eta\right)\right|+\mathcal{O}\left(N^{2 \epsilon} \kappa_{0}\right),
$$

where we used Lemma 4.2 Hence, using (A.23), we obtain for $v_{i} \notin \Sigma_{\epsilon}$ that

$$
\begin{aligned}
\frac{1}{\left|\lambda v_{i}-z-m_{f c}(z)\right|^{2}} & \leq \frac{1}{\left|\lambda v_{i}-\left(L_{+}+\mathrm{i} \eta\right)-m_{f c}\left(L_{+}+\mathrm{i} \eta\right)\right|^{2}}+\frac{N^{2 \epsilon} \kappa_{0}}{\left|\lambda v_{i}-\left(L_{+}+\mathrm{i} \eta\right)-m_{f c}\left(L_{+}+\mathrm{i} \eta\right)\right|^{3}} \\
& \leq \frac{1+C N^{-\epsilon}}{\left|\lambda v_{i}-\left(L_{+}+\mathrm{i} \eta\right)-m_{f c}\left(L_{+}+\mathrm{i} \eta\right)\right|^{2}} .
\end{aligned}
$$

We thus have that

$$
\begin{aligned}
\frac{1}{N} & \sum_{i=2}^{N} \frac{1}{\left|\lambda v_{(i)}-z-m_{f c}(z)\right|^{2}} \\
& \leq \frac{N^{3 \epsilon(\mathrm{b}+2)}}{N} \frac{1}{\left(N^{-\epsilon} \kappa_{0}\right)^{2}}+\frac{1}{N} \sum_{i: v_{i} \notin \Sigma_{\epsilon}} \frac{1+C N^{-\epsilon}}{\left|\lambda v_{i}-\left(L_{+}+\mathrm{i} \eta\right)-m_{f c}\left(L_{+}+\mathrm{i} \eta\right)\right|^{2}} \\
& \leq N^{-\epsilon}+\frac{1}{N} \sum_{i=1}^{N} \frac{1+C N^{-\epsilon}}{\left|\lambda v_{i}-\left(L_{+}+\mathrm{i} \eta\right)-m_{f c}\left(L_{+}+\mathrm{i} \eta\right)\right|^{2}}<c<1,
\end{aligned}
$$

where we also used the assumption that $\left|v_{(2)}-v_{(1)}\right| \geq N^{-\epsilon} \kappa_{0}$.

(2) When $E>L_{+}+N^{2 \epsilon} \kappa_{0}$, we have

$$
\left(E-L_{+}\right)+\left(\operatorname{Re} m_{f c}(E+\mathrm{i} \eta)-\operatorname{Re} m_{f c}\left(L_{+}+\mathrm{i} \eta\right)\right) \gg \eta+\operatorname{Im} m_{f_{c}}(E+\mathrm{i} \eta),
$$

where we again used Lemma 4.2, hence, from (A.23) we obtain that

$$
\left|\lambda v_{i}-z-m_{f c}(z)\right| \geq\left|\lambda v_{i}-\left(L_{+}+\mathrm{i} \eta\right)-m_{f c}\left(L_{+}+\mathrm{i} \eta\right)\right| .
$$

We may now proceed as in (1) to find that

$$
\begin{aligned}
& \frac{1}{N} \sum_{i=1}^{N} \frac{1}{\left|\lambda v_{i}-z-m_{f c}(z)\right|^{2}} \\
& \quad \leq N^{-\epsilon}+\frac{1}{N} \sum_{i=1}^{N} \frac{1}{\left|\lambda v_{i}-\left(L_{+}+\mathrm{i} \eta\right)-m_{f c}\left(L_{+}+\mathrm{i} \eta\right)\right|^{2}}<c<1 .
\end{aligned}
$$

Since we proved in Lemma A.1 that the assumptions $N^{-\epsilon} \kappa_{0}<\left|1-v_{(1)}\right|<(\log N) \kappa_{0}$ and $\left|v_{(1)}-v_{(2)}\right|>N^{-\epsilon} \kappa_{0}$ hold with probability higher than $1-C(\log N)^{1+2 \mathrm{~b}} N^{-\epsilon}$, we find that the desired lemma holds for any $z \in \mathcal{D}_{\epsilon}^{\prime}$.

To conclude this appendix, we prove Lemma A.4.

Proof of Lemma A.4. We start with the claim (1): Notice that

$$
\operatorname{Im} m_{f c}(z)=\operatorname{Im} \int \frac{\mathrm{d} \mu_{f c}(x)}{x-z}=\eta \int \frac{\mathrm{d} \mu_{f c}(x)}{\left(x-L_{+}+\kappa\right)^{2}+\eta^{2}} .
$$

When $\eta \geq 1 / 2$, we may use the trivial bound

$$
\operatorname{Im} m_{f c}(z) \sim \eta \int \frac{\mathrm{d} \mu_{f c}(x)}{\eta^{2}}=\eta^{-1} \sim 1 \sim\left(\kappa^{\beta}+\eta\right) .
$$

When $\kappa \geq 1 / 2$, we can easily see from (A.27) that $\operatorname{Im} m_{f c}(z) \sim 1 \sim\left(\kappa^{\beta}+\eta\right)$. Thus, in the following, we only consider the case $\kappa, \eta<1 / 2$.

To prove the lower bound, we notice that

$$
\operatorname{Im} m_{f c}(z) \geq \eta \int_{L_{+}-\kappa-2 \eta}^{L_{+}-\kappa-\eta} \frac{\mathrm{d} \mu_{f c}(x)}{\left(x-L_{+}+\kappa\right)^{2}+\eta^{2}} \geq C \eta \int_{L_{+}-\kappa-2 \eta}^{L_{+}-\kappa-\eta} \frac{(\kappa+\eta)^{\beta} \mathrm{d} x}{\eta^{2}} \geq C(\kappa+\eta)^{\beta} \geq C \kappa^{\beta} .
$$

We also have that

$$
\operatorname{Im} m_{f c}(z) \geq \eta \int_{0}^{1} \frac{\mathrm{d} \mu_{f c}(x)}{\left(x-L_{+}+\kappa\right)^{2}+\eta^{2}} \geq C \eta \int_{0}^{1} \mathrm{~d} x \geq C \eta
$$


Thus, we find that $\operatorname{Im} m_{f c}(z) \geq C\left(\kappa^{\beta}+\eta\right)$.

For the upper bound, we first consider the case $\kappa \geq \eta$, where we have

$$
\begin{aligned}
\operatorname{Im} m_{f c}(z) & \leq C \eta \int_{L_{-}}^{L_{+}-\kappa-\eta} \frac{\left(L_{+}-x\right)^{\beta} \mathrm{d} x}{\left(x-L_{+}+\kappa\right)^{2}}+C \eta \int_{L_{+}-\kappa-\eta}^{L_{+}-\kappa+\eta} \frac{(\kappa+\eta)^{\beta} \mathrm{d} x}{\eta^{2}}+C \eta \int_{L_{+}-\kappa+\eta}^{L_{+}} \frac{\kappa^{\beta} \mathrm{d} x}{\left(x-L_{+}+\kappa\right)^{2}} \\
& \leq C \eta \int_{\eta}^{L_{+}-L_{-}-\kappa} \frac{(y+\kappa)^{\beta} \mathrm{d} y}{y^{2}}+C(\kappa+\eta)^{\beta}+C \eta \int_{\eta}^{\kappa} \frac{\kappa^{\beta}}{y^{2}} \mathrm{~d} y \\
& \leq C(\kappa+\eta)^{\beta}+C \eta \\
& \leq C\left(\kappa^{\beta}+\eta\right) .
\end{aligned}
$$

Here, we used that $(y+\kappa)^{\beta} \leq C\left(y^{\beta}+\kappa^{\beta}\right)$. The calculation for the case $\kappa<\eta$ is similar, in fact, easier.

We now prove the claim (2). As in the proof of the statement (1), we only consider the case $\kappa, \eta<1 / 2$. We have, for the lower bound, that

$$
\operatorname{Im} m_{f c}(z) \geq \eta \int_{0}^{1} \frac{\mathrm{d} \mu_{f c}(x)}{\left(x-L_{+}-\kappa\right)^{2}+\eta^{2}} \geq C \eta \int_{0}^{1} \mathrm{~d} x \geq C \eta,
$$

and, for the upper bound,

$$
\operatorname{Im} m_{f c}(z) \leq C \eta \int_{L_{-}}^{L_{+}} \frac{\left(L_{+}-x\right)^{\beta} \mathrm{d} x}{\left(x-L_{+}-\kappa\right)^{2}} \leq C \eta \int_{\kappa}^{L_{+}-L_{-}+\kappa} \frac{y^{\beta}}{y^{2}} \mathrm{~d} y \leq C \eta .
$$

This completes proof of the desired lemma.

\section{Appendix B}

In this appendix, we prove Lemma 6.4 and Lemma 6.5

Proof of Lemma 6.4. Set for $l, l^{\prime} \in \llbracket 0, p \rrbracket$ and for $A=\llbracket n_{0}, N \rrbracket$,

$$
\Gamma_{l, l^{\prime}}(z) \equiv \Gamma_{l, l^{\prime}}:=\max \left\{\left|F_{a b}^{\left(\mathbb{T}, \mathbb{T}^{\prime}\right)}(z)\right|: a, b \in A, a \neq b, \mathbb{T}, \mathbb{T}^{\prime} \subset A,|\mathbb{T}| \leq l,\left|\mathbb{T}^{\prime}\right| \leq l^{\prime}\right\}
$$

For simplicity we drop the $z$-dependence from the notation and always work on $\Omega_{V}$. We first consider $\Gamma_{l, 0}$, i.e., we set $\mathbb{T}^{\prime}=\emptyset$. Recalling that $\mathbb{1}(\Xi)\left|F_{a b}(z)\right| \leq\left(\varphi_{N}\right)^{c \xi} N^{-\mathfrak{b}} N^{\epsilon}$, we obtain $p \Gamma_{0,0} \leq p\left(\varphi_{N}\right)^{c \xi} N^{-\mathfrak{b}+\epsilon} \ll 1$ on $\Xi$. From $(\underline{6.5})$, we get, for $c \in A$,

$$
F_{a b}^{(\mathbb{T} c, \emptyset)}=F_{a b}^{(\mathbb{T}, \emptyset)}-F_{a c}^{(\mathbb{T}, \emptyset)} F_{c b}^{(\mathbb{T}, \emptyset)}
$$

and we obtain on $\Xi$,

$$
\Gamma_{l+1,0} \leq \Gamma_{l, 0}+\Gamma_{l, 0}^{2}
$$

Iterating (B.3), we obtain

$$
\Gamma_{l+1,0} \leq \Gamma_{0,0}+\sum_{i=0}^{l} \Gamma_{i, 0}^{2} \leq \Gamma_{0,0}+\left(l \Gamma_{l, 0}\right) \Gamma_{l+1,0}
$$

Thus as long as $l \Gamma_{0,0} \leq 1 / 4$, we obtain by induction on $l, \Gamma_{l+1,0} \leq 2 \Gamma_{0,0}$, on $\Xi$, proving (6.21) for the special case $\mathbb{T}^{\prime}=\emptyset$. To prove the claim for $\mathbb{T}^{\prime} \neq \emptyset$, we fix $l=|\mathbb{T}|$ and observe that $(6.5)$, together with the assumption $\Gamma_{l, l^{\prime}} \ll 1$, implies

$$
\Gamma_{l, l^{\prime}+1} \leq \Gamma_{l, l^{\prime}}+C \Gamma_{l, l^{\prime}}^{2}
$$

for some numerical constant $C$. Iterating, we find

$$
\Gamma_{l, l^{\prime}+1} \leq \Gamma_{l, 0}+C \sum_{i^{\prime}=0}^{l^{\prime}} \Gamma_{l, i^{\prime}}^{2} \leq \Gamma_{l, l^{\prime}}+C l \Gamma_{l, l^{\prime}} \Gamma_{l, l^{\prime}+1} .
$$

Thus as long as $C l^{\prime} \Gamma_{l, l^{\prime}} \leq 1 / 4$, we obtain on $\Xi$ that $\Gamma_{l, l^{\prime}+1} \leq 2 \Gamma_{l, 0} \leq 4 \Gamma_{0,0}$, where we used that $\Gamma_{l, 0} \leq 2 \Gamma_{0,0}\left(\leq N^{-\mathfrak{b}}\right)$. This proves (6.21). 
To prove (6.22), we define, for $l \in \llbracket 1, p \rrbracket$,

$$
\widetilde{\Gamma}_{l}:=\max \left\{\left|\frac{F_{a b}^{(\emptyset, a)}}{G_{a a}^{(\mathbb{T})}}\right|: a, b \in A, a \neq b, \mathbb{T} \subset A, a \notin \mathbb{T},|\mathbb{T}| \leq l\right\} .
$$

Note that $\widetilde{\Gamma}_{0} \leq\left(\varphi_{N}\right)^{c \xi} N^{-1 / 2+2 \epsilon}$, on $\Xi$. From (6.7), we have on $\Xi$

$$
\widetilde{\Gamma}_{l+1} \leq \widetilde{\Gamma}_{l}+C \widetilde{\Gamma}_{l} \Gamma_{l, l}^{2},
$$

for a numerical constant $C$. Iterating as above, we find

$$
\widetilde{\Gamma}_{l+1} \leq \widetilde{\Gamma}_{0}+C \sum_{i=0}^{l} \widetilde{\Gamma}_{i} \Gamma_{i, i}^{2} \leq \widetilde{\Gamma}_{0}+8 C \Gamma_{0,0}^{2} \sum_{i=0}^{l} \widetilde{\Gamma}_{i}
$$

Since $8 C l \Gamma_{0,0}^{2} \leq 1 / 4$, on $\Xi$, we obtain $\widetilde{\Gamma}_{l+1} \leq 2 \widetilde{\Gamma}_{0}$, on $\Xi$. Upon using $(6.21)$ to bound $\left|F_{a b}^{(\emptyset, a)}-F_{a b}^{\left(\mathbb{T}^{\prime}, \mathbb{T}^{\prime \prime}\right)}\right|$, this proves (6.22).

The proof of (6.23) is similar to the proof of (6.22) but easier.

Proof of Lemma 6.5. First, we observe that for a random variables $\mathcal{X} \equiv \mathcal{X}(H)$,

$$
\mathbb{E}^{W}\left|Q_{b} \mathcal{X}\right|^{p}=\mathbb{E}^{W}\left|\mathcal{X}-\mathbb{E}_{b} \mathcal{X}\right|^{p} \leq 2^{p-1} \mathbb{E}^{W}|\mathcal{X}|^{p}+2^{p-1} \mathbb{E}^{W}\left|\mathbb{E}_{b} \mathcal{X}\right|^{p}
$$

From Jensen's inequality for the partial expectation $\mathbb{E}_{b}$, we hence obtain

$$
\mathbb{E}^{W}\left|Q_{b} \mathcal{X}\right|^{p} \leq 2^{p} \mathbb{E}^{W}|\mathcal{X}|^{p}
$$

Next, let $h_{i}:=2\left\lceil\frac{2+p}{1+d_{i}}\right\rceil, i \in \llbracket 1, p \rrbracket$. One checks that $\sum_{i=1}^{p} h_{i}^{-1} \leq 1,2 \leq h_{i} \leq 2 p+4$ and $h_{i}\left(d_{i}+1\right) \leq 2 p+4$. Thus Hölder's inequality gives

$$
\left|\mathbb{E}^{W} \prod_{i=1}^{q} Q_{i} \mathcal{X}_{i} \prod_{i=q+1}^{p} Q_{i} \mathcal{Y}_{i}\right| \leq 2^{q} \prod_{i=1}^{q}\left(\mathbb{E}^{W}\left|\mathcal{X}_{i}\right|^{h_{i}} \mid\right)^{1 / h_{i}} \prod_{i=q+1}^{p}\left(\mathbb{E}^{W}\left|Q_{i} \mathcal{Y}_{i}\right|^{h_{i}}\right)^{1 / h_{i}}
$$

where we used (B.11). By assumption, we have

$$
\mathbb{E}^{W}\left[\left|\mathcal{X}_{i}\right|^{h_{i}} \mathbb{1}\left(\Xi^{c}\right)\right] \leq\left(\mathbb{E}^{W}\left|\mathcal{X}_{i}\right|^{2 h_{i}}\right)^{1 / 2} \mathbb{P}\left(\Xi^{c}\right)^{1 / 2} \leq N^{2 K h_{i}\left(d_{i}+1\right)} \mathbb{P}\left(\Xi^{c}\right)^{1 / 2} \ll \mathrm{e}^{-c(\log N)^{\xi}} .
$$

Observing that $\frac{1}{h_{i}} \geq \frac{1+d_{i}}{4 p}$, we thus get

$$
\begin{aligned}
\left(\mathbb{E}^{W}\left|\mathcal{X}_{i}\right|^{h_{i}} \mathbb{1}\left(\Xi^{c}\right)\right)^{1 / h_{i}} & \leq \mathrm{e}^{-c \frac{1+d_{i}}{4 p}(\log N)^{\xi}} \\
& \leq\left(\mathrm{e}^{-c(\log N)^{3 / 2}}\right)^{1+d_{i}} \\
& \ll N^{-\left(1+d_{i}\right)},
\end{aligned}
$$

where we used that $p^{-1} \geq(\log N)^{-\xi+3 / 2}$. In a similar way, one establishes

$$
\left(\mathbb{E}^{W}\left|\mathcal{Y}_{i}\right|^{h_{i}} \mathbb{1}\left(\Xi^{c}\right)\right)^{1 / h_{i}} \ll N^{-1} .
$$

Together with the estimates (6.24), valid on the event $\Xi$, we obtain

$$
\left|\mathbb{E}^{W} \prod_{i=1}^{q} Q_{i} \mathcal{X}_{i} \prod_{i=q+1}^{p} Q_{i} \mathcal{Y}_{i}\right| \leq\left(\varphi_{N}\right)^{c_{0} \xi p} N^{-\sum_{i=1}^{q}\left(d_{i}-1\right)(\mathfrak{b} / 2-\epsilon)} N^{2 p \epsilon} .
$$

Recalling that we have set $\sum_{i=1}^{q} d_{i}-1=s$, the claim follows.

\section{Appendix C}

In this appendix, we consider the setup $\lambda<\lambda_{+}$. Recall that $\widehat{\mu}_{f c}=\widehat{\mu} \boxplus \mu_{s c}$ denotes the free convolution measure of the empirical measure $\widehat{\mu}$, which is defined in (4.13), and the semicircular measure $\mu_{s c}$. Also recall that we denote by $L_{ \pm}$the endpoints of the support of the measure $\mu_{f c}$. 
Lemma C.1. Let $\mu$ be a centered Jacobi measure defined in (2.10) with $\mathrm{b}>1$. Let $\operatorname{supp} \widehat{\mu}_{f c}=\left[\widehat{L}_{-}, \widehat{L}_{+}\right]$, where $\widehat{L}_{-}$ and $\widehat{L}_{+}$are random variables depending on $\left(v_{i}\right)$. Then, if $\lambda<\lambda_{+}$, the rescaled fluctuation $N^{1 / 2}\left(\widehat{L}_{+}-L_{+}\right)$converges to a Gaussian random variable with mean 0 and variance $\left(1-\left[m_{f c}\left(L_{+}\right)\right]^{2}\right)$ in distribution, as $N \rightarrow \infty$.

Remark C.2. When a > 1, the analogous statement to Lemma C.1 holds at the lower edge. See also Remark 2.6 . Proof. Following the proof in [44, 32, we find that $\widehat{L}_{+}$is the solution to the equations

$$
\widehat{m}_{f c}\left(\widehat{L}_{+}\right)=\frac{1}{N} \sum_{j=1}^{N} \frac{1}{\lambda v_{j}-\widehat{L}_{+}-\widehat{m}_{f c}\left(\widehat{L}_{+}\right)}, \quad \frac{1}{N} \sum_{j=1}^{N} \frac{1}{\left(\lambda v_{j}-\widehat{L}_{+}-\widehat{m}_{f_{c}}\left(\widehat{L}_{+}\right)\right)^{2}}=1 .
$$

Similarly, we find that $L_{+}$is the solution to the equations

$$
m_{f c}\left(L_{+}\right)=\int \frac{\mathrm{d} \mu(v)}{\lambda v-L_{+}-m_{f c}\left(L_{+}\right)}, \quad \int \frac{\mathrm{d} \mu(v)}{\left(\lambda v-L_{+}-m_{f c}\left(L_{+}\right)\right)^{2}}=1 .
$$

Let

$$
\tau:=L_{+}+m_{f c}\left(L_{+}\right), \quad \widehat{\tau}:=\widehat{L}_{+}+\widehat{m}_{f c}\left(\widehat{L}_{+}\right) .
$$

Since $\lambda<\lambda_{+}$, we may assume that

$$
\int \frac{\mathrm{d} \mu(v)}{(\lambda v-\lambda)^{2}}>1+\delta, \quad \frac{1}{N} \sum_{j=1}^{N} \frac{1}{\left(\lambda v_{j}-\lambda\right)^{2}}>1+\delta,
$$

for some $\delta>0$. Notice that the second inequality holds with high probability on $\Omega_{V}$. From the assumption, we also find that $\tau, \widehat{\tau}>\lambda$. Thus we get

$$
\begin{aligned}
0 & =\frac{1}{N} \sum_{j=1}^{N} \frac{1}{\left(\lambda v_{j}-\widehat{\tau}\right)^{2}}-1=\frac{1}{N} \sum_{j=1}^{N} \frac{1}{\left(\lambda v_{j}-\widehat{\tau}\right)^{2}}-\frac{1}{N} \sum_{j=1}^{N} \frac{1}{\left(\lambda v_{j}-\tau\right)^{2}}+\mathcal{O}\left(\left(\varphi_{N}\right)^{\xi} N^{-1 / 2}\right) \\
& =\frac{1}{N} \sum_{j=1}^{N} \frac{\left(-2 \lambda v_{j}+\tau+\widehat{\tau}\right)(\tau-\widehat{\tau})}{\left(\lambda v_{j}-\tau\right)^{2}\left(\lambda v_{j}-\widehat{\tau}\right)^{2}}+\mathcal{O}\left(\left(\varphi_{N}\right)^{\xi} N^{-1 / 2}\right)
\end{aligned}
$$

which holds with high probability. Since $\tau, \widehat{\tau}>\lambda$, we have

$$
-2 \lambda v_{j}+\tau+\widehat{\tau} \geq 0 .
$$

Moreover, with high probability, $\left|\left\{v_{j}: v_{j}<0\right\}\right|>c N$ for some constant $c>0$, independent of $N$. In particular,

$$
\frac{1}{N} \sum_{j=1}^{N} \frac{-2 \lambda v_{j}+\tau+\widehat{\tau}}{\left(\lambda v_{j}-\tau\right)^{2}\left(\lambda v_{j}-\widehat{\tau}\right)^{2}}>c^{\prime}>0
$$

for some constant $c^{\prime}$ independent of $N$. This shows together with (C.4) that

$$
\tau-\widehat{\tau}=\mathcal{O}\left(\left(\varphi_{N}\right)^{\xi} N^{-1 / 2}\right),
$$

with high probability on $\Omega_{V}$.

We can thus write

$$
\begin{aligned}
\widehat{m}_{f c}\left(L_{+}\right)=\widehat{\tau}-\widehat{L}_{+} & =\frac{1}{N} \sum_{j=1}^{N} \frac{1}{\lambda v_{j}-\widehat{\tau}}=\frac{1}{N} \sum_{j=1}^{N} \frac{1}{\lambda v_{j}-\tau}+\frac{1}{N} \sum_{j=1}^{N} \frac{\widehat{\tau}-\tau}{\left(\lambda v_{j}-\tau\right)^{2}}+\mathcal{O}\left(\left(\varphi_{N}\right)^{2 \xi} N^{-1}\right) \\
& =m_{f c}\left(L_{+}\right)+X+(\widehat{\tau}-\tau)+\mathcal{O}\left(\left(\varphi_{N}\right)^{2 \xi} N^{-1}\right),
\end{aligned}
$$

with high probability, where we define the random variable $X$ by

$$
X:=\frac{1}{N} \sum_{j=1}^{N} \frac{1}{\lambda v_{j}-\tau}-\int \frac{\mathrm{d} \mu(v)}{\lambda v-\tau}=\frac{1}{N} \sum_{j=1}^{N}\left(\frac{1}{\lambda v_{j}-\tau}-\mathbb{E}\left[\frac{1}{\lambda v_{j}-\tau}\right]\right) .
$$

Notice that, by the central limit theorem, $X$ converges to a Gaussian random variable with mean 0 and variance $N^{-1}\left(1-\left(m_{f c}\left(L_{+}\right)\right)^{2}\right)$. Since,

$$
L_{+}-\widehat{L}_{+}=X+\mathcal{O}\left(\left(\varphi_{N}\right)^{2 \xi} N^{-1}\right),
$$

with high probability, the desired lemma follows. 
When $\left(v_{i}\right)$ are fixed, we may follow the proof of Theorem 2.21 in 32 to get

$$
\left|L_{+}-\mu_{1}\right| \leq\left(\varphi_{N}\right)^{C \xi} N^{-2 / 3}
$$

with high probability. Since $\left|\widehat{L}_{+}-L_{+}\right| \sim N^{-1 / 2}$, we find that the leading fluctuation of the largest eigenvalue comes from the Gaussian fluctuation obtained in Lemma C.1. This also shows that there is a sharp transition in the distribution of the largest eigenvalue from a Gaussian law to a Weibull distribution as $\lambda$ crosses $\lambda_{+}$.

\section{REFERENCES}

[1] Aizenman, M., Molchanov, S.: Localization at Large Disorder and at Extreme Energies: An Elementary Derivation Commun. Math. Phys. 157, 245-278 (1993).

[2] Anderson, G. W., Guionnet, A., Zeitouni, O.: An Introduction to Random Matrices, Cambridge University Press (2010).

[3] Auffinger, A., Ben Arous, G., Péché, S.: Poisson Convergence for the Largest Eigenvalues of Heavy Tailed Random Matrices, Ann. Inst. Henri Poincaré - Probab. Stat. 45, 589-610 (2009).

[4] Belinschi, S. T., Benaych-Georges, F., Guionnet, A.: Regularization by Free Additive Convolution, Square and Rectangular Cases, Complex Analysis and Operator Theory 3, 611-660 (2009).

[5] Belinschi, S. T., Bercovici, H.: A New Approach to Subordination Results in Free Probability, J. Anal. Math., 101, 357-365 (2007).

[6] Belinschi, S. T., Bercovici, H., Capitaine, M., Février, M.: Outliers in the Spectrum of Large Deformed Unitarily Invariant Models, arXiv:1207.5443 (2012).

[7] Biane, P.: On the Free Convolution with a Semi-circular Distribution, Indiana Univ. Math. J. 46, 705-718 (1997).

[8] Bordenave, C., Guionnet, A.: Localization and Delocalization of Eigenvectors for Heavy-tailed Random Matrices, Probab. Theory Relat. Fields 157, 885-953 (2013)

[9] Capitaine, M., Donati-Martin, C., Féral, D., Février, M.: Free Convolution with a Semi-circular Distribution and Eigenvalues of Spiked Deformations of Wigner Matrices, Electron. J. Probab. 16, 1750-1792 (2011).

[10] Bouchaud, J.-P., Cizeau, P.: Theory of Lévy matrices, Phys. Rev. E 3, 1810-1822, (1994).

[11] Chistyakov, G.P., Götze, F.: The Arithmetic of Distributions in Free Probability Theory, Cent. Euro. J. Math. 9, 997-1050, (2011).

[12] Erdős, L.: Universality of Wigner Random Matrices: A Survey of Recent Results, arXiv:1004.0861v2 (2010).

[13] Erdős, L., Knowles, A.: Quantum Diffusion and Eigenfunction Delocalization in a Random Band Matrix Model, Commun. Math. Phys. 303, 509-554 (2011).

[14] Erdős, L., Knowles, A., Yau, H.-T., Yin, J.: Spectral Statistics of Erdős-Rényi Graphs I: Local Semicircle Law, Ann. Probab. 41, 2279-2375 (2013).

[15] Erdős, L., Knowles, A., Yau, H.-T., Yin, J.: Spectral Statistics of Erdős-Rényi Graphs II: Eigenvalue Spacing and the Extreme Eigenvalues, Commun. Math. Phys. 314, 587-640 (2012).

[16] Erdős, L., Knowles, A., Yau, H.-T., Yin, J.: Delocalization and Diffusion Profile for Random Band Matrices, arXiv:1205.5669v3 (2012).

[17] Erdős, L., Knowles, A., Yau, H.-T., Yin, J.: The Local Semicircle Law for a General Class of Random Matrices, Electr. J. Prob. 18, no.59, 1-58 (2013).

[18] Erdős, L., Knowles, A., Yau, H.-T.: Averaging Fluctuations in Resolvents of Random Band Matrices, arXiv:1205.5664 (2012).

[19] Erdős, L., Ramirez, J., Schlein, B., Yau, H.-T.: Universality of Sine-kernel for Wigner Matrices with a Small Gaussian Perturbation, Electr. J. Prob. 15, no.18, 526-603 (2010). 
[20] Erdős, L., Schlein, B., Yau, H.-T.: Semicircle Law on Short Scales and Delocalization of Eigenvectors for Wigner Random Matrices, Ann. Probab. 37, 815-852 (2009).

[21] Erdős, L., Schlein, B., Yau, H.-T.: Local Semicircle Law and Complete Delocalization for Wigner Random Matrices, Commun. Math. Phys. 287, 641-655 (2009).

[22] Erdős, L., Schlein, B., Yau, H.-T.: Wegner Estimate and Level Repulsion for Wigner Random Matrices, Int. Math. Res. Notices. 2010, 436-479 (2010).

[23] Erdős, L., Yau, H.-T., Yin, J.: Bulk Universality for Generalized Wigner Matrices, Probab. Theory Relat. Fields 154, 341-407 (2012).

[24] Erdős, L., Yau, H.-T., Yin, J.: Universality for Generalized Wigner Matrices with Bernoulli Distribution, J. Comb. 2, 15-82 (2012).

[25] Erdős, L., Yau, H.-T., Yin, J.: Rigidity of Eigenvalues of Generalized Wigner Matrices, Adv. Math. 229, 1435-1515 (2012).

[26] Fröhlich, J., Spencer, T.: Absence of Diffusion in the Anderson Tight Binding Model for Large Disorder or Low Energy, Commun. Math. Phys. 88, 151-184 (1983).

[27] Haan, L. d. L., Ferreira, A.: Extreme value theory, Springer, (2006).

[28] Hiai, F., Petz, D.: The Semicircle Law, Free Random Variables and Entropy, American Mathematical Society (2006).

[29] Johansson, K.: From Gumbel to Tracy-Widom, Probab. Theory Relat. Fields 138, 75-112 (2007).

[30] Kargin, V.: Subordination of the Resolvent for a Sum of Random Matrices, arXiv:1109.5818 (2011).

[31] Minami, N.: Local Fluctuation of the Spectrum of a Multidimensional Anderson Tight Binding Model, Commun. Math. Pysics. 177, 709-725 (1996).

[32] Lee, J. O., Schnelli, K.: Local Deformed Semicircle Law and Complete Delocalization for Wigner Matrices with Random Potential, J. Stat. Phys. 54103504 (2013).

[33] Lee, J. O., Yin, J.: A Necessary and Sufficient Condition for Edge Universality of Wigner Matrices, To appear in Duke Math. J., arXiv:1206.2251 (2012).

[34] Novak, J., LaCroix, M.: Three Lectures on Free Probability, arXiv:1205.2097 (2012).

[35] Nica, A., Speicher, R.: Lectures on the Combinatorics of Free Probability, Cambridge University Press (2006).

[36] Olver, S., Nadakuditi, R. R.: Numerical Computation of Convolutions in Free Probability Theory, arXiv:1203.1958 (2013).

[37] O'Rourke, S., Vu, V.: Universality of Local Eigenvalue Statistics in Random Matrices with External Source, arXiv:1308.1057 (2013).

[38] Pastur, L.: On the Spectrum of Random Matrices, Theor. Math. Phys. 10, 67-74 (1972).

[39] Pastur, L., Vasilchuk, V.: On the Law of Addition of Random Matrices, Commun. Math. Phys. 214, 249-286 (2000).

[40] Péché, S., Soshnikov, A.: On the Lower Bound of the Spectral Norm of Symmetric Random Matrices with Independent Entries, Electron. Commun. Probab. 13, 280290 (2008).

[41] Péché, S., Soshnikov, A.: Wigner Random Matrices with Non-Symmetrically Distributed Entries, J. Stat. Phys. 129, 857884 (2007).

[42] Schenker, J., Eigenvector Localization for Random Band Matrices with Power Law Band Width, Commun. Math. Phys. 290, 1065-1097 (2009).

[43] Shcherbina, T.: On universality of Bulk Local Regime of the Deformed Gaussian unitary ensemble, Math. Phys. Anal. Geom. 5, 396-433 (2009).

[44] Shcherbina, T.: On Universality of Local Edge Regime for the Deformed Gaussian Unitary Ensemble, J. Stat. Phys. 143, 455-481 (2011). 
[45] Sinai, Y., Soshnikov, A.: A Refinement of Wigners Semicircle Law in a Neighborhood of the Spectrum Edge, Functional Anal. and Appl. 32, 114131 (1998).

[46] Soshnikov, A.: Universality at the Edge of the Spectrum in Wigner Random Matrices, Commun. Math. Phys. 207, 697-733 (1999).

[47] Soshnikov, A.: Poisson Statistics for the Largest Eigenvalue of Wigner Random Matrices with Heavy Tails, Elect. Commun. in Probab. 9, 82-91 (2004).

[48] Tao, T., Vu, V.: Random Matrices: Universality of Local Eigenvalue Statistics up to the Edge, Commun. Math. Phys. 298, 549-572 (2010).

[49] Tracy, C., Widom, H.: Level-Spacing Distributions and the Airy Kernel, Commun. Math. Phys. 159, 151-174 (1994).

[50] Tracy, C., Widom, H.: On Orthogonal and Symplectic Matrix Ensembles, Commun. Math. Phys. 177, 727-754 (1996).

[51] Voiculescu, D., Dykema, K. J., Nica, A.: Free Random Variables: A Noncommutative Probability Approach to Free Products with Applications to Random Matrices, Operator Algebras and Harmonic Analysis on Free Groups, American Mathematical Society (1992).

[52] Wigner, E. P.: Characteristic Vectors of Bordered Matrices with Infinite Dimensions, Ann. Math. 62, 548-564 (1955). 